STUDIES

.

in

AFRIGAN LINGUISTICS

MARCH 1978

Volume 9 , Number 1

Published by the Department of Linguistics and the African Studies Center University of California, Los Angeles 
Published by the Department of Linguistics and the African Studies Center

The University of California, Los Angeles

Editorial Board

Baruch Elimelech

Victoria Fromikin

Talmy Givon

Jean-Marie Hombert

Mazisi Kunene

Peter Ladefoged

Carol Lord

Thomas Penchoen

Paul Schachter

Yero Sylla

Benji Wald

William E. Weimers

Joint Editors

Thomas J. Hinnebusch

Russell G. Schuh
Associate Editors

John Eulenberg, Michigan State

Robert Hetzron, UCSB

Larry Hyman, USC

Leon C. Jacobson, Maiduguri

William R. Leben, Stanford

Martin Mould, USC

Paul Newman, Leiden

Thomas H. Peterson, CSULA

Carol Myers Scotton, Michigan State

Herbert Stahlke, Georgia State

Erhard Voeltz, Honolulu, Hawaii

Editorial Assistance

Alice McGaughey

John Singler

Ann West

Subscription: Individuals: $\$ 8.00$ per volume

Institutions: $\quad \$ 12.00$ per volume

Single issues: $\$ 4.00$

Supplements: $\quad \$ 8.00$ (if ordered separately or in back order)

(Air mail rates are available upon request)

Make checks payable to: African Studies Center

Studies in African linguistics is published three times a year.

Supplements are independently financed and are sent free of charge with current subscriptions. Authors receive 25 free offprints of published articles. Papers submitted to the journal should be sent in triplicate.

For subscriptions and editorial information, please write:

The Editors, Studies in African Linguistics

Department of Linguistics

University of California

Los Angeles, CA 90024

Volume 9, Number 1, March 1978

Copyright 1978

By the Regents of the University of California 


\author{
Volume 9, Number 1 \\ March 1978
}

TABLE OF CONTENTS

\title{
Articles
}

Robert Nicolaï, LES PARLERS SONGHAY OCCIDENTAUX (TOMBOUCTOU - JENNÉ NGORKU . . . . . . . . . . . . . . . . . . . . . . 1

Antoinette Oomen, FOCUS IN THE RENDILLE CLAUSE . . . . . . . . . 35

Papers from the 8th Conference on African Linguistics

Afroasiatic Working Group

Philip Jaggar, 'AND WHAT ABOUT ...?' - TOPICALISATION IN HAUSA . . .

Greta D. Little, WORD ORDER FUNCTION TYPOLOGY: THE AMHARIC CONNECTION . . . . . . . . . . . . . . . . . . . . . .

Bantu Syntax Working Group

Salikoko S. Mufwene, A RECONSIDERATION OF LINGALA TEMPORAL INFLECTIONS . . . . . . . . . . . . . . . . . . . . . . .

Susan U. Stucky, LOCATIVE PHRASES AND ALTERNATIVE CONCORD IN TSHILUBA . . . . . . . . . . . . . . . . . . . . . 107 


\author{
LES PARLERS SONGHAY OCCIDENTAUX \\ (TOMBOUCTOU - JENNE - NGORKU) \\ Robert Nicolai \\ Université de Niamey
}

\begin{abstract}
This article consists of two parts: the first is a description of the phonological system of the variant of Songhai spoken in Timbuktu. The Timbuktu dialect can be differentiated from the other Songhai dialects by the absence of the phoneme $/ z /$ and by an analysis which leads to the discovery of prenasalized consonants in the system. In the process of presenting the description, we also discuss the methodology which has guided this analysis. We discuss in detail such concepts as "correlation of correlations", "degrees of correlation" and "pseudocorrelation", which we have found useful in formulating the description. The second part, which is strictly descriptive, takes into account the differences we have established between the variants spoken in Timbuktu, Jenne and $N^{\prime} G o r k u$. We present these differences in the form of a series of tables regarding: variations in post-vocalic consonants, conditioned variations between /v/ and /w/, /s/ variations, combinations of $r+$ apical consonant, and the distribution of the phoneme $/ s /$. These are the main points of difference among these dialect variants on the phonematic level, and appear to be so slight as to have hardly any repercussions on the interpretation of the system.
\end{abstract}

\title{
1. Présentation Générale
}

Le songhay, langue que Greenberg a classé dans le groupe Nilosaharien et que les auteurs précédents avaient considéré soit comme une langue isolée soit comme une langue appartenant à la même famille que les langues mandé, a été peu décrit du point de vue phonologique. Cette "langue" se divise en plusieurs dialectes tels que le songhay proprement dit, parlé surtout au Mali et un peu au Niger; le zarma, parlé exclusivement au Niger; le dendi, parlé un peu au Niger, au Nord du Bénin et probablement en quelques autres points isolés de la Nigéria. Toutefois cette classification, qui donne les "grandes lignes" de la diversification dialectale, est néanmoins insuffisante et doit être reprise de manière beaucoup plus élaborée pour rendre compte de 
la réalité linguistique de la région. Ce ne sera cependant pas l'objet de cet article. Nous allons nous attacher ici à la description phonématique de l'un des "sous-dialectes" du songhay, à savoir celui de la région de Tombouctou. I En effet, le songhay "malien" que nous venons de mentionner se divise au moins en trois sous-ensembles: celui de Gao, celui de Tombouctou et celui de Hombori. Ce dernier, bien que parlé par peu de personnes, n'en est pas le moins intéressant. Cependant les dialectes (ou sous-dialectes) de Tombouctou et de Gao sont considérés comme les plus importants, lorsque $I^{\prime}$ on se réfère au nombre de locuteurs qui les parlent. C'est d'ailleurs à ces deux dialectes que l'on pense généralement lorsqu'il est fait référence aux parlers songhaỹ "malien". La frontière entre eux deux se si.tuerait, d'après Prost [1956], ${ }^{2}$ près d'Arnasey à $25 \mathrm{~km}$ à l'est de Tombouctou.

Cette division en deux dialectes est clairement ressentie par les songhayphones eux-mêmes car à la fois la syntaxe, la morphologie et la phonologie en sont très différentes de l'un à l'autre. A l'intérieur même du dialecte "tombouctien" nous nous appercevrons vite qu'il, existe un certain nombre de dif'érences, ce qui, dans la mesure où nous décrirons certaines de ces variations, explique notre titre: les parlers songhay occidentaux (Tombouctou-Jenné-Ngorku), lequel indique quels sont les parlers que nous avons pris en considération.

Nous nous attacherons à l'aspect phonologique de ces parlers et nous traiterons essentiellement de la phonématique. Les faits prosodiques seront décrits ultérieurement, dans le cadre d'un travail d'ensemble sur le songhay que nous avons entrepris depuis quelques années. D'une façon plus pratique, nous présenterons d'abord les données du parler de Tombouctou et c'est ensuite, de façon contrastive, que nous présenterons les autres parlers étudiés, à savoir: celui de Jenné

${ }^{1} C^{\prime}$ est ce parler que décrit Dupuis-Yakouba [1917]. Cet ouvrage comprend en outre un lexique de la langue.

${ }^{2}$ L'auteur signale "une opposition nette entre le groupe situé à l'ouest et au sud-ouest de Tombouctou (en amont ou plus exactement d'Arnasey, et ceux situés à l'est" [Prost 1956]. 
et celui de Ngorku ce dernier représentant une variante parlée non loin de Niafounké dans le Jimbala (région du delta intérieur).

Tout au long de cet article notre propos sera non pas de faire une présentation uniforme mais plutôt, après une courte présentation générale des points n'offrant pas de difficultés particulières, de traiter de certains aspects d'analyse qui demandent une solution motivée. En particulier nous traiterons du problème de l'existence ou de la non-existence des consonnes prénasalisées, du statut de la nasalité et de la labialité dans ces parlers, puis celui de l'organisation des corrélations.

Le travail sur Tombouctou a été fait en partie auprès d'un ensemble d'immigrés tombouctiens résidant à Niamey; il en est de même du travail sur Jenné. Ensuite tous ces travaux ont été vérifiés sur place, au cours de missions de recherche au Mali. Les données sur le parler de Ngorku ont été recueillies sur place.

\section{Les Voyelles}

2.1. Apercu du système vocalique tombouctien. Nous avons affaire à un système triangulaire à trois degrés, déterminé par l'opposition de longueur. La pertinence des oppositions de degré et de localisation peutêtre établie à partir des paires minimales dans (1) et (2). Ces oppositions sont possibles dans les monosyllabes, ainsi que l'attestent les exemples, et dans presque toutes les positions.

(1) Voyelles en position finale ouverte

$\begin{array}{llllll}i \sim e & \text { ci } & \text { 'parler' } & \text { ce } & \text { 'pied' } \\ e \sim \text { a } & \text { me } & \text { 'bouche' } & \text { ma } & \text { 'nom' } \\ a \sim 0 & \text { ma } & \text { 'nom' } & \text { mo } & \text { 'oeil' } \\ \circ \sim u & \text { yo } & \text { 'chameau' } & \text { yu } & \text { 'abeille' } \\ i \sim u & j i & \text { 'beurre' } & \text { ju } & \text { 'bande de tissu' } \\ e \sim 0 & \text { me } & \text { 'bouche' } & \text { mo } & \text { 'oeil' }\end{array}$

En syllabe ouverte non-finale l'opposition de longueur se réalise pour tous les degrés et pour toutes localisations. 
(2) Voyelle en syllabe initiale, position non-absolue (\#C__CV ...)

\begin{tabular}{|c|c|c|c|c|c|c|}
\hline \multirow[t]{6}{*}{ a. } & $\sim$ & e: & $j i: r i$ & 'frotter' & je:ri & 'terrasser' \\
\hline & $\sim$ & $\mathrm{a}:$ & je:ri & 'terrasser' & ja:ri & 'jour' \\
\hline & $\sim$ & ०: & ha: bu & 'coton' & ho: bu & 'canari' \\
\hline & $\sim$ & $\mathrm{u}:$ & go:ru & 'vallée' & $g u: r u$ & 'fer' \\
\hline & $\sim$ & $\mathrm{u}:$ & ni:ne & 'nez' & nu: ne & 'feu' \\
\hline & $\sim$ & ०: & & & & \\
\hline \multirow{6}{*}{ b. i } & v & e & biri & 'os' & bere & 'défricher' \\
\hline & $\sim$ & a & herey & 'famine' & harey & 'tambourin' \\
\hline & $\sim$ & $\circ$ & harey & 'tambourin' & horey & 'amusement' \\
\hline & $\sim$ & $u$ & kosu & 'effeuiller' & kusu & 'marmite' \\
\hline & $\sim$ & u & & & & \\
\hline & $\sim$ & $\mathrm{e}$ & horey & 'amusement' & herey & 'famine' \\
\hline \multirow[t]{2}{*}{ c. } & $\sim$ & ०: & go: ro & 'cola' & goro & 's'asseoir' \\
\hline$u$ & $\sim$ & $\mathrm{u}:$ & $k u: \int e$ & $\begin{array}{l}\text { 'estomac' } \\
\text { d'animal }\end{array}$ & kusu & 'marmite' \\
\hline$\theta$ & $\sim$ & e: & be: re & 'grand père' & bere & 'défricher' \\
\hline $\mathrm{i}$ & $\sim$ & $\mathrm{i}:$ & $f i: s i$ & 'balayer' & fisi & 'huilé' \\
\hline & $\sim$ & $\mathrm{a}:$ & fa:ta & 'âne gris' & fata & 'aile' \\
\hline
\end{tabular}

En position initiale absolue nous n'avons relevé qu'un inventaire réduit, soit $i, a, u$ :

(3) Voyelle en position initiale absolue (\#__ CV ...)

$\begin{array}{lll}\text { ije 'fils' } & \text { isa } & \text { 'fleuve' } \\ \text { ura } & \text { 'or' } & \text { ayu }\end{array}$

L'opposition de Iongueurn'est pas réalisée en syllabe finale ouverte, elle n'apparaît pas non plus en position initiale absolue; par contre elle apparait dans n'importe quelle autre position et il semble possible de la dégager en syllabe fermée, ce qui n'est pas le cas pour tous les parlers songhay. Exemple:
(4) $\quad \operatorname{tam}$
'esclave'
ta:m
'soulier'
$\operatorname{tar}$
'fade'
ta: $r$
'toucher'
igay' [gøy] 'Iuminère, tarder'
ga:y
'poisson, esp.' 
2.2. Voyelle suivie d'un élément post-vocalique (_C $\left.C\left\{\begin{array}{l}\mathrm{CV} \\ \#\end{array}\right\}\right)$.

L'inventaire semble également réduit quand la voyelle est suivie d'une semi-voyelle appartenant à la même syllabe, mais dans les autres cas de syllabe fermée par une consonne liquide ou bien par une consonne nasale nous n'avons pas relevé de perte de distinctivité. L'ensemble des attestations des combinaisons relevées entre une voyelle et une consonne post-vocalique peut être présenté dans les tables ci-dessous:

(5) Voyelle suivie d'un élément post-vocalique (_C $\left.\left\{\begin{array}{l}\mathrm{CV} \\ \#\end{array}\right\}\right)$

\begin{tabular}{|c|c|c|c|c|c|}
\hline & $\mathrm{i}$ & $\mathrm{e}$ & a & 0 & $u$ \\
\hline & $X$ & $\mathrm{X}$ & $X$ & $\mathrm{X}$ & $X$ \\
\hline & & $\mathrm{X}$ & $X$ & $X$ & $X$ \\
\hline & $\mathrm{X}$ & $\mathrm{X}$ & $X$ & & $\mathrm{X}$ \\
\hline
\end{tabular}

$\begin{array}{llllll}\text { tar 'fade' } & \text { ham } & \text { 'viande' } & \text { man } & \text { 'où' } \\ \text { ber 'grand' } & \text { hem } & \text { 'pleurer' } & \text { ben } & \text { 'finir' } \\ \text { tor 'fétiche' } & \text { kom } & \text { 'brigander' } & \text { hun } & \text { 'quitter' } \\ \text { dur 'piler' } & \text { tum } & \text { 'percer' } & \text { din } & \text { 'bruler' }\end{array}$

bibir 'filer'

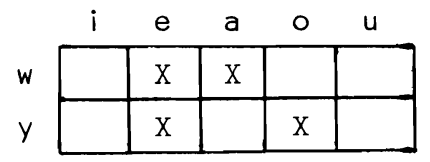

$\begin{array}{lll}\text { haw 'vache' hoy } & \text { 'sauce' } \\ \text { hew 'vent' } & \text { dey }[d \varepsilon y] \text { 'acheter' }\end{array}$

Le cas offert par ces combinaisons entre voyelles et semi-voyelles, seul cas où l'inventaire est réduit, est intéressant en ce qu'il pose un problème d'interprétation. Nous le présentons ci-dessous en motivant notre choix et ses implications. Nous avons phonétiquement les attestations suivantes:

$$
\begin{aligned}
& \text { [oy] hoy 'sauce'; goy 'travail' }
\end{aligned}
$$

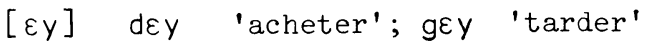

$$
\begin{aligned}
& \text { [ay] hay 'enfanter' } \\
& \text { [a:y] ga:y 'poisson (esp.)' }
\end{aligned}
$$


(6) cont.

$$
\begin{aligned}
& \text { [ew] hew 'vent'; yew 'étranger' } \\
& {\left[\begin{array}{l}
0 w] \\
\left.a_{w}\right]
\end{array}\right\} \text { gow 'carrie' }} \\
& \text { [aw] haw 'vache' }
\end{aligned}
$$

Le problème consiste à savoir quelles sont exactement les unités représentées par les sons vocaliques devant les semi-voyelles. La première constatation d'évidence est que le degré fermé a disparu; il ne reste que le sous-système $\varepsilon, \curvearrowright, a$. Une étude attentive des données nous permet d'établir que l'opposition $a \sim \varepsilon$, dans le contexte __ $y$, et l'opposition $\supset \sim a$, dans le contexte _ $W^{W}$, ne sont pas clairement établies; il est facile de remarquer que [a] n'apparaît qu'après $/ \mathrm{h} /$ et que, de ce fait, il y a une complémentarité entre les deux réalisations.

Soit donc une opposition de deux voyelles dans chacun des contextes en question:
(7) 0 ,
$\varepsilon / a \quad / \quad y$
e, s/a / w w

Un problème d'identification se pose: que représentent respectivement $\varepsilon / a$ et $\lrcorner / a$ ? Deux solutions peuvent être envisagées:

(a) Soit ces deux réalisations représentent respectivement $/ \varepsilon /$ et $/ \mathrm{J} /$ et nous avons alors l'opposition [antérieur] [postérieur] comme seule opposition vocalique. Le phonème /a/ ou plutôt la classe qu'il représente n'est pas réalisée dans ce contexte.

Système complet

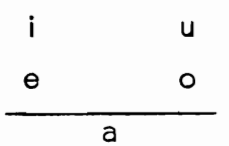

Système réduit

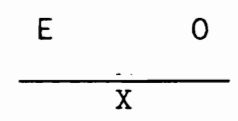

Cette hypothèse se heurte à une première difficulté qui est l'existence de [a:] dans le contexte _y, par exemple, [ga:y] 'poisson (esp. de ...)' parallèle à $[t a: r]$ 'toucher'; [ta:m] 'soulier'. Nous pourrions admettre que ce $[a:]$, dans ce contexte, représente le phonème /a/ et nous aurions ainsi: 
$\left(8^{\prime}\right)$

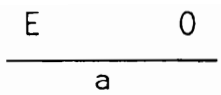

Toutefois nous émettons quelques réserves à partir des constatations suivantes:

- les unités [hay] et [ga:y] seraient interprétées /hEy/ et/gay/;

- les unités [ga:y] et [†a:m] seraient interprétées /gay/ et / †a:m/.

Cette différence d'interprétation de la longueur vocalique ne nous semble pas justifiée, puisqu'il s'avère par ailleurs que l'opposition /a/ /a:/ existe dans la langue, par exemple, tam 'esclave' ta:m 'soulier'. L'interprétation de [hay] comme/hEy/ ne se justifie pas non plus, puisque l'on admet l'existence de /a/ dans ce contexte.

D'autre part, si [hay] est interprété comme/hay/, il faudra dire que: /-y/ a un "pouvoir allongeant" sur le phonème /a/ qui le précède et /h/ un "pouvoir abrégeant" sur ce même phonème, ce qui ne simplifierait rien.

Nous proposons done de refuser cette solution.

(b) Soit les deux réalisations $\varepsilon / a$ et $s / a$ représentent respectivement le phonème /a/.

(9)

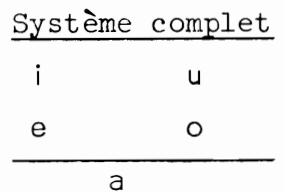

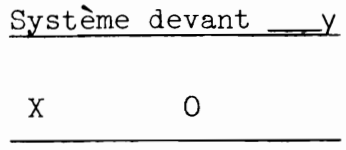

a
Système devant _. W

$\frac{E-X}{a}$

L'absence de Ey et de Ow s'explique par une règle de contrainte (dissimilation). Une combinaison, appartenant à une même syllabe, définie par les traits: [+ vocalique] + [+ vocalique], introduit un accord tel

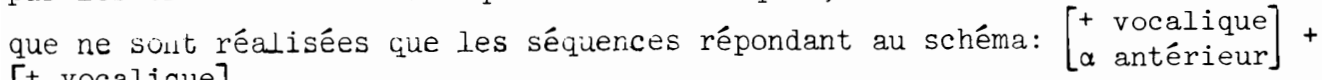
$\left[\begin{array}{c}+ \\ -\alpha \text { antérieur }\end{array}\right]$.

Les réalisations de /a/ qui tendent vers [ع] ou [o] s'expliquent à leur tour par le contexte:

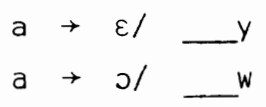

Ces réalisations mêmes sont rendues possibles par l'absence de l'opposition: 
$\begin{array}{ccc}\nu & \sim & a / \\ \varepsilon & \sim & a / \ldots\end{array}$

La réalisation [ga:y] s'oppose à [gey]. La séquence /ga:y/ s'oppose à /gay/ de la même manière que la séquence /ta:r/ s'oppose à /tar'. Nous remarquerons, avec cette hypothèse, que compte tenu de la complémentarité de $E / \ldots$ sidérer $E$ et $O$ comme deux variantes contextuelles, ce qui amènerait à un système réduit basé sur la seule opposition [ouvert] [non ouvert]. (10) $E / O \sim a$

Cette solution est équivalente à celle qui a été présentée ci-dessus, quant à son résultat. Elle implique que ce qui était présenté comme une contrainte soit présenté comme une neutralisation. Nous ne choisissons pas cette interprétation car nous avons admis la neutralisation sur la dimension [fermé non fermé]; cette dernière interprétation conduirait à admettre une nouvelle neutralisation sur la dimension [antérieur non antérieur], soit une fois sur une dimension d'aperture et une autre fois sur une dimension de localisation. Or l'opposition $0 \sim a$ et $E \sim a$ est aussi une opposition d'aperture, laquelle n'est pas neutralisée. Nous ne considérerions l'éventualité d'une neutralisation entre /0/ et /E/ qu'après la perte totale des oppositions d'aperture, ce qui n'est pas le cas. C'est pourquoi nous préférons parler de contrainte plutôt que de neutralisation, eut égard à une certaine conception hiérarchisée des phénomènes.

2.3. Conclusion sur le système vocalique. L'inventaire maximal des phonèmes vocaliques est le suivant:

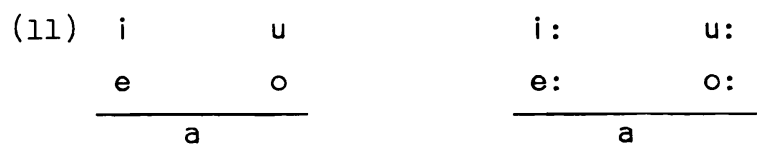

Il n'est jamais réalisé au contact de la frontière du mot (initiale absolue et/ou finale ouverte); et il est réduit dans le contexte d'une semi-voyelle post-vocalique, ainsi que nous l'avons vu. 


\section{Les Consonnes}

3.1. Aperçu du système consonantique. L'inventaire des phonèmes consonantiques est le suivant, $(p), t, c, k, b, d, j, g, f, s, h, m, n, r$, $\eta, r, I, w, Y$. Un problème se pose quant à I'interprétation des consonnes "prénasalisées", mp, mb, nt, nd, nc, nj, jk, ng, et suivant l'analyse, jS, mf. Nous essaierons, dans la suite du texte, de donner une solution à ce problème, après avoir présenté quelques attestations pour illustrer les diverses oppositions.

(12) Consonnes en position prévocalique initiale

$(\#+\mathrm{V}(:) \mathrm{CV} \ldots)$

Points d'articulation

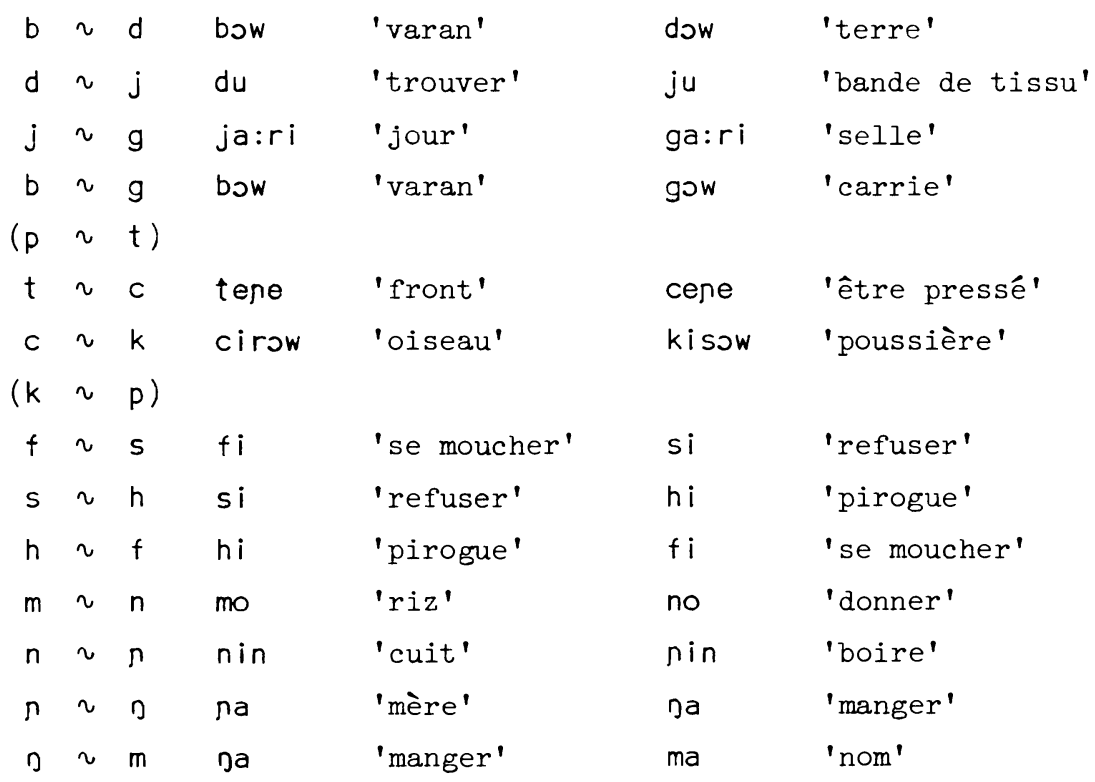

Nasalité

$\begin{array}{llllll}b & \sim m & \text { ba } & \text { 'aimer' } & \text { ma } & \text { 'nom' } \\ d \sim n & \text { do } & \text { 'endroit' } & \text { no } & \text { 'donner' } \\ j \sim n & \text { jin } & \text { 'devancer' } & \text { nin } & \text { 'boire' } \\ g & \sim n & \text { ga } & & \text { na } & \text { 'manger' }\end{array}$


Occlusivité

$\begin{array}{llllll}b & \sim f & \text { bi } & \text { 'hier' } & \mathrm{fi} & \text { 'se moucher' } \\ t \sim s & \text { to } & \text { 'arriver' } & \text { so } & \text { 'filtre' } \\ c \sim s & \text { cendu } & \text { 'tirer' } & \text { sendu } & \text { 'dur' } \\ j & \sim s & \text { samba } & \text { 'envoyer' } & \text { jamba } & \text { 'trahir' } \\ k \sim h & k u & \text { 'Iong' } & \text { hu } & \text { 'maison' } \\ g & \sim h & \text { gani } & \text { 'pou' } & \text { hani } & \text { 'poisson' }\end{array}$

Palatalité

$\begin{array}{llllll}c & \sim s & c i & \text { 'parler' } & \text { si } & \text { 'refuser' } \\ j \sim s & j i & \text { 'nager' } & \text { si } & \text { 'refuser' } \\ y \sim j & y \varepsilon w & \text { 'étranger' } & \text { jहw } & \text { 'porter' } \\ y \sim s & \text { yo } & \text { 'chameau' } & \text { so } & \text { 'filtre' } \\ y \sim c & y \varepsilon w & \text { 'étranger' } & \text { cहw } & \text { 'lire' }\end{array}$

Apicalité

\begin{tabular}{|c|c|c|c|c|c|}
\hline$d \sim$ & $n$ & do & 'endroit' & no & 'donner' \\
\hline$\sim$ & I & do & 'endroit' & 10 & 'Iécher' \\
\hline$\sim$ & 1 & no & 'donner' & 10 & 'lécher' \\
\hline
\end{tabular}

Labialité

b $\sim w$ bow 'varan' wow 'insulte'

Cet inventaire permet d'attester la pertinence des oppositions présentées. Il faut cependant remarquer que:

(a) /p/ n'apparaît que dans de rares unités, généralement empruntées. De ce fait il n'a pas été possible de dégager de paires minimales pour illustrer les oppositions $p \sim b, p \sim t, p \sim f$, mais aucune contrainte distributionnelle ne permet de rejeter le phonème /p/ qui, à un certain niveau d'analyse, peut être présenté dans l'inventaire des phonèmes. C'est le sens qu'il faut donner aux parenthèses qui l'entourent.

(13) Attestations de $/ p /$

\begin{tabular}{|c|c|c|c|}
\hline pat i & 'couper' & pendel & 'jupe, petit pagne' \\
\hline senne & 'voter, donner sa confiance' & jippa & 'plonger' \\
\hline lempe & 'bande de tissu' & npo:gi & 'slip' \\
\hline
\end{tabular}

(b) L'opposition $r \sim 1$ n'a pas été illustrée parce qu'elle n'est jamais réalisée au contact de la pause, c'est-à-dire ni en initiale ni 
en finale de mot. Nous trouvons généralement [l] en initiale et $[r]$ en finale. Par contre, en position interne, il est loisible d'établir cette opposition.

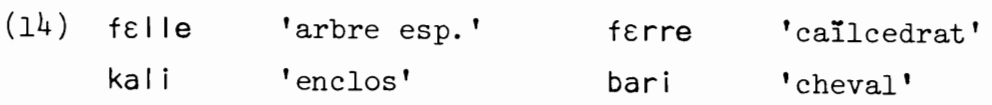

Compte tenu de cette restriction, l'ensemble des consonnes présentées est possible en n'importe quelle position prévocalique.

3.2. Consonnes en position post-vocalique. L'inventaire des phonèmes, dans cette position, est réduit. Nous commencerons par l'étude de la position finale absolue. L'inventaire est le suivant $r, m, n, w, y$, b , (s). Des attestations illustrant les combinaisons entre voyelles et sonantes ont déjà été données avec les tables dans (5).

Nous présentons ci-dessous quelques paires minimales pour attester la valeur phonématique des unités présentées.

$\begin{array}{llllll}\text { (15) } m & \sim n & \text { ham } & \text { 'viande' } & \text { han } & \text { 'jour' } \\ w & \sim y & \text { gaw [gow] } & \text { 'carrie' } & \text { gay }[g \varepsilon y] & \text { 'tarder' } \\ b & \sim w & \text { ceb } & \text { 'heurter' } & \text { cew } & \text { 'lire' }\end{array}$

Ceci fait nous sommes obligés, pour rendre correctement compte du système consonantique, de traiter des combinaisons intervocaliques. Cela nous amènera à:

a) Statuer sur l'existence des consonnes dites prénasalisées.

b) Statuer sur le comportement des consonnes bruyantes et continues.

c) Statuer sur les consonnes occlusives labiales.

d) Statuer sur la structure syllabique.

Ces différentes opérations sont nécessaires si l'on tient à rendre compte de l'organisation du système et à présenter quelque chose qui ne soit pas une simple taxinomie d'unités phonématiques. Des donnés complémentaires necéssaires à l'analyse se trouvent dans (16) - (18).

(16) Consonnes prénasalisées initiales

\begin{tabular}{|c|c|c|c|c|}
\hline npogi & 'slip' & $D S$ & ogorfu & 'croix de malte' \\
\hline ntenda & 'fourmi rouge' & $n j$ & njono & 'chacal' \\
\hline ncom & 'rat' & nd & nduna & 'monde' \\
\hline nkondo & 'fourmi' & $\mathrm{mb}$ & mbala & 'crapaud' \\
\hline
\end{tabular}


(17) Combinaisons de consonnes intervocaliques avec un premier élêment nasal
a. $m p$
nt sint $i$
'commencer'
$\mathrm{mb}$
simbal
'poisson (esp.)'
no hancin
'chèvre'
nd
sundu
'priser'
ok haykam
'être serré'
nj genji
'fourche'
ng gungu
'île cultivée'
b. ynd hoyndi 'invitation' rnd berndi 'transformer'
(wnd)
( Ind)
c. mf hamfi 'grand vase en terre'
ns hapdi 'chien'
funsu 'souffler'
d. nt ramti 'pincer' ms demsu 'mettre en plus'
ma kamdi 'pousser pour mn hamni 'mouche'

(18) Combinaisons de consonnes intervocaliques avec un premier élément occlusif

$\begin{array}{lll}\text { bd } & \text { jibda } & \text { 'encens' } \\ \text { bt } & \text { libti } & \text { 'navet' } \\ \text { bk } & \text { sibka } & \text { 'broderie' } \\ \text { bs } & \text { cibsi } & \text { 'tabaski' }\end{array}$

Nous avons maintenant assez d'éléments pour aborder l'analyse.

3.2.1. Recherche d'une interprétation des données. Nous trouvons un paradigme initial de consonnes définies comme prénasalisées du point de vue phonétique et ces mêmes unités sont réalisables en position intervocalique; d'autre part, l'existence de combinaisons $-\left\{\begin{array}{l}W \\ y\end{array}\right\}+N+C-$, qui n'apparaissent jamais en position finale, nous amènent à tracer la frontière syllabique comme suit:

$$
\left\{\begin{array}{l}
w \\
y
\end{array}\right\} \quad N C
$$

Nous sommes donc conduits à admettre l'existence de consonnes prénasalisées dans le parler considéré ${ }^{3}$ et le système occlusif se présente alors sous la

${ }^{3}$ Nous avons refusé l'existence des consonnes prénasalisées dans le parler zarma de Dosso où les conditions n'étaient pas comparables. Une discussion sur ce point se trouve dans Nicolaï [1976]. 
forme suivante d'apparence très régulière:

(20) $\begin{array}{cccc}(p) & t & c & k \\ b & d & j & g \\ (n p) & n t & n c & n k \\ n b & n d & n j & n g \\ m & n & n & n\end{array}$

Cette interprétation a pour conséquence de réduire considérablement le nombre des groupes consonantiques intervocaliques, puisque tous ceux qui sont présentés en (17a) ne sont plus analysés comme tels mais comme les occurrences des phonèmes prénasalisés. Les seules combinaisons d'un élément nasal avec une consonne suivante restent:

$\begin{array}{lll}\text { (2l) } & m & f, s \\ n & s, \\ m & t, d, s, n\end{array}$

Soit donc deux combinaisons homorganiques $\mathrm{mf}$, ns, les autres combinaisons se définissant au travers d'un schéma [labial] + [apical]. Les deux combinaisons homorganiques [mf] et [ns] (ou peut être mieux [us]) peuvent être interprétées comme des phonèmes prénasalisés, à l'instar des occlusives, par généralisation à partir de l'analyse précédente et bien que nous ne les trouvions pas en position initiale absolue.

Cette interprétation s'avère la plus simple: elle permet d'interdire la combinaison de toute consonne nasale autre que /m/ en position intervocalique et de simplifier les règles de combinaison des unités occlusives en les insérant dans le schèma [labial] + [apical]. Une autre solution, dans laquelle [mf] et [ns] seraient interprétés comme la combinaison du /n/ qui est relevé en finale absolue avec les deux consonnes [non occlusives] / f/ et /s/ n'a pas été retenue, dans la mesure où elle n'apportait aucune simplification.

Les combinaisons d'un élément nasal avec une consonne suivante restent donc: $m$. $s, t, d, n$. Nous avons maintenant suffisamment avancé l'analyse pour aborder un problème important et corrélatif de celui que nous traitons, à savoir l'étude des combinaisons de /b/ avec une consonne subséquente où, plus globalement, l'étude du fonctionnement des consonnes labiales dans le système. 
3.2.2. Etude succincte de la corrélation labiale. Nous avons noté "entre parenthèses" les phonèmes /p/ et / $\mathrm{np} /$, en soulignant qui'ils n'appartenaient pas au même niveau d'analyse que les autres phonèmes consonantiques du système. L'absence de l'occlusive labiale sourde est caractéristique de nombreuses langues dans lesquelles cette absence ne représente pas une "case vide" mais possède au contraire un sens structural. C'est le cas du songhay où le phonème /p/ne s'intègre pas facilement et peut, le cas échéant, être remplacé par $[f]$, où une distribution particulière du phonème / b/ conduit ce dernier à fonctionner différemment des autres phonèmes [occlusif] [oral]. Nos remarques vont porter sur les points suivants: /b/ possède une distribution qui lui est propre et qui est la même que celle de /m/; ces deux unités, auxquelles on adjoindra /mb/, compte-tenu de leurs particularités fonctionnelles formeront une corrélation des consonnes labiales. Ainsi le rapport $b \sim d$ ne sera pas de même nature que le rapport $d \sim j$ et le rapport $m \sim n$, ne sera pas de même nature que le rapport $n \sim n$.

Nous établissons un premier état de description où, à partir de l'hypothèse sur l'existence d'une corrélation des consonnes labiales déterminée par un fonctionnement homogène, nous pouvons dresser le tableau suivant qui tient compte de ce fonctionnement: ${ }^{4}$

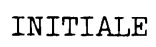

m

mb

b

\section{FINALE}

m

$$
-
$$

b

\section{INTERVOCALIQUE}

$\begin{array}{ll}\text { mn } & - \\ \text { ms } & \text { bs } \\ m t & \text { bt } \\ \text { md } & \text { bd }\end{array}$

Ce tableau nous présente les positions où sont attestées ces consonnes ainsi que les combinaisons qu'elles acceptent.

${ }^{4}$ L'étude du particularisme des consonnes labiales dans les parlers songhay a été traité dans Nicolaĩ [1977] où le problème est envisagé de facon plus générale et plus approfondie. 
Le phonème /mb/ n'est pas réalisé en position post-vocalique où nous avons un phénomène de neutralisation. Deux possibilités d'interprétation nous sont offertes: soit admettre une neutralisation entre $/ \mathrm{m} /$ et $/ \mathrm{mb} /$, soit admettre une neutralisation entre $/ \mathrm{mb} /$ et $/ \mathrm{b} /$. Notre choix sera déterminé par les caractéristiques fonctionnelles des unités concernées: /m/ est possible devant une consonne nasale (cf: -mn-), mais $\mathrm{ni} / \mathrm{b} / \mathrm{ni} / \mathrm{mb} / \mathrm{ne}$ sont possibles dans ce contexte. Nous nous appuyerons sur ce fait pour regrouper ensemble /b/ et /mb/ et admettre que la neutralisation a lieu entre ces deux phonèmes plutôt qu'entre $/ \mathrm{m} /$ et $/ \mathrm{mb} /$.

De par l'existence de cette neutralisation, notre corrélation labiale du départ se scinde en deux corrélations distinctes, déterminées par la dimension [oral], soit:

- corrélation [labial et oral]: $\quad\{m b, b\}$

- corrélation [labial et non oral]: $\{\mathrm{m}\}$

Cette dernière corrélation est réduite à une seule unité. Dans la corrélation "orale" c'est la "série" [oral et nasal] (cf. /mb/) qui est marquée par rapport à la "série" [oral et non nasal], dans la mesure où /mb/ n'apparait jamais dans la position de neutralisation.

Ce rapprochement que nous venons de faire entre la consonne prénasalisée et la consonne orale recoupe l'analyse que l'on est amené à faire à propos des consonnes non-labiales, soit la division en deux corrélations de l'ensemble des phonèmes occlusifs à partir de la dimension [oral], compte tenu du comportement particulier des consonnes nasales: $n t, n c, n k$, nd, $n j, n g, t, c, k, d, j, g$ en face de $n, n, n$. La corrélation des nasales et la corrélation des occlusives orales constituent bien des corrélations distinctes puisqu'elles fonctionnent de manière différente.

4. Remarques d'ordre Terminologique: Considerations sur la Notion de Corrélation

Nous avons ci-dessus accordé une grande importance au concept de corrélation et, du fait même de cette importance, il est nécessaire de spécifier ce que recouvre pour nous ce terme afin d'éviter toute ambiguité. Toutefois, le lecteur intéressé par les seules analyses du songhay, pourra directement se reporter à 5.1 . 
4.1. Corrélation. Une corrélation se définit pour nous, à la fois par une identité de comportement syntagmatique et par l'existence d'un rapport corrélatif entre les unités de la corrélation; les deux critères sont nécessaires à la définition. Le rapport corrélatif, qui est évident quand on peut dégager deux séries, comme dans l'ensemble

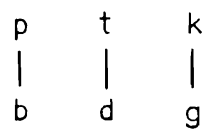

semble plus difficile à établir si la corrélation "unité fonctionnelle" ne permet de regrouper qu'une série, comme c'est souvent le cas pour les nasales $m \quad n \quad D$. Dans ce cas nous supposons que le rapport corrélatif existe toujours, mais qu'il s'applique sur lui-même. La neutralisation n'est que l'une des particularités de fonctionnement susceptible d'affecter une corrélation. Les définitions suivantes seront également utiles à notre analyse.

4.2. Sous-corrélation. Une sous-corrélation est une structure qui possède la même structure phonique que la corrélation à laquelle elle se rattache, mais qui constitue en elle-même une série de cette corrélation, c'est-à-dire un sous-ensemble qui est dans un rapport corrélatif avec l'autre série de la corrélation. Par exemple en songhay où nous avons à la fois des occlusives sourdes, sonores et prénasalisées, si toutes ces consonnes fonctionnent de la même manière sans qu'un fait de neutralisation rompe la symétrie de leur comportement, nous aurons à établir des sous-corrélations:

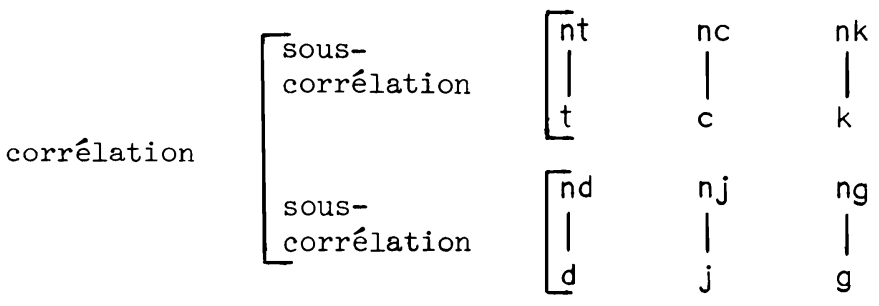

4.3. Corrélation de corrélations. Toutefois, s'il s'introduit une différence de comportement entre les deux sous-corrélations, alors celles-ci 
vont se constituer en corrélations distinctes. Cescorrélations distinctes pourront être regroupées dans une corrélation de corrélation. C'est le cas par exemple des deux corrélations labiales du songhay $m$ et $\mathrm{mb}, \mathrm{b}$ qui se différencient par l'existence d'une neutralisation affectant la deuxième et que nous regroupons dans une corrélation de corrélation des consonnes labiales. La raison de ce regroupement est évidente dans la mesure où ces deux corrélations ont la même distribution.

Cette hiérarchisation permet de rendre compte de ce qui est commun aux deux corrélations, en les regroupant dans cette unité fonctionnelle plus large, tout en soulignant ce qui les différencie. Nous reprenons ainsi, du point de vue fonctionnel, le concept de "faisceau de corrélations" présenté par A. Martinet.

4.4 Corrélations phonetiques ou pseudo-corrélations. Ceci dit, il peut être utile de caractériser d'autres rapports de la langue. Si nous prenons en songhay l'exemple que nous fournissent la corrélation occlusive orale, $n d, d, n t, t .$. , et la corrélation occlusive nasale, $n, n, \eta$, qui ne fonctionnent pas du tout de la même manière et $n^{\prime}$ ont pas la même distribution, nous voyons - au moins au niveau de la phonétique distinctive - qu'il existe entre ces deux corrélations une différence identique à celle que nous pouvons établir entre les corrélations $m b$, b et $m$ qui, elles, constituent ensemble une corrélation de corrélation; seulement, dans le cas qui nous occupe, cette différence phonétique n'est pas liée à une caractéristique fonctionnelle précise.

Nous proposons donc de regrouper les phonèmes liés par un tel rapport phonétique dans une unité que nous appellerons pseudo-corrélation. Une pseudo-corrélation sera définie par l'existence, entre certaines unités, d'un rapport phonétique utilisé par ailleurs dans la même langue pour opposer les séries d'une même corrélation ou les corrélations d'une même corrélation de corrélation. Nous pensons que le rapport qui s'établit entre une corrélation et une pseudo-corrélation doit être productif dans l'explication des phénomènes d'évolution linguistique. Ce rapport fait apparaitre la tension qui existe entre l'organisation structurale abstraite de la langue et sa réalisation dans le matériau phonétique. 
5. Description de l'Analyse

Une fois données ces précisions terminologiques, nous allons mener l'analyse à partir d'une succession de choix (de divisions) opérés sur les dimensions phoniques de la langue; ces successions de choix illustreront la hiérarchisation introduite dans l'analyse des faits; elles seront faites en tenant compte de nos réflexions précédentes.

5.1. Analyse du système consonantique. Nous proposons une première division de l'ensemble des consonnes sur la dimension [sonant], laquelle regroupe d'un côté toutes les classes de phonèmes réalisables en position postvocalique, de l'autre côté toutes celles qui ne sont pas réalisables dans cette position, soit:

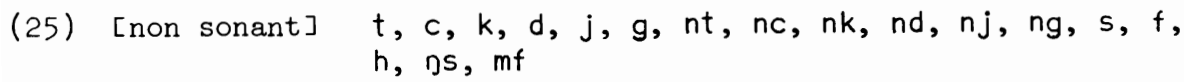
[sonant]

[sonant] et [non sonant] sont des traits catégoriels ${ }^{5}$ liés à des positions syntagmatiques déterminées. Dans le cadre [non sonant] une nouvelle division s'établira sur la dimension [sonorisable]. ${ }^{6}$ En effet nous avons vu que les phonèmes de la corrélation $f, s, h, m f$, $n s{ }^{7}$ n'ont pas de corrélats sonores; de plus, ces phonèmes ont la propriété de ne pas pouvoir se géminer, comme c'est le cas pour les phonèmes des autres corrélations. ${ }^{8}$ Nous remarquons que la dimension [continu]

${ }^{5}$ Un trait catégoriel est un trait qui caractérise une catégorie; une catégorie est définie par l'existence d'un position syntagmatique donnée, ce qui est différent d'une corrélation où l'identité de distribution doit être valable pour toutes les positions syntagmatiques où apparaît l'ensemble de phonèmes.

${ }^{6}$ [sonorisable] est plutôt une dimension relationnelle que substantielle, dans la mesure où elle permet d'opposer les unités qui peuvent être soit sourdes ou soit sonores à celles qui n'ont pas cette caractéristique. Toutefois, l'importance de ce trait nous est clairement apparue dans l'étude fonctionnelle; nous l'avons déjà utilisé dans Nicolaĩ [1976].

${ }^{7} L^{\prime}$ absence de / $\mathrm{h} /$ relève à notre avis d'une contrainte particulière et n'est pas le fait d'une régularité structurale.

${ }^{8}$ Quelques géminations s'entendent parfois, mais nous croyons quelles sont dues à l'influence des parlers voisins; de même nous trouvons [guffa] touffe de cheveu rituelle, mais il s'agit d'une attestation isolée d'un vocabulaire spécialisé et à notre avis peu courant. 
est liée à la dimension [sonorisable] de telle manière que les phonèmes [non sonorisable] soient toujours [continu]. Toutefois, nous remarquerons que les consonnes prénasalisées ne sont jamais géminées non plus. Comme nous avons déjà établi l'existence d'une neutralisation entre /b/ et /mb/ dans la position postrocalique (on trouve $m b \sim b$ en position initiale, mais on ne trouve que $b$ en position finale) nous nous appuyons sur ce fait pour généraliser la conclusion dans le cadre des phonèmes [non sonant]: les phonèmes définis à la fois comme [oral] et [nasal] sont considérés comme étant marqué par rapport aux autres phonèmes corrélatifs. Ainsi on aurait $\mathrm{mb}, \mathrm{b} ; \mathrm{nd}$, $d$ en position initiale. En position intervocalique, alors que sont possibles les combinaisons bb; dd, les combinaisons mmb et nnd ne sont pas réalisables. Nous pouvons, pour I'instant, établir la hiérarchie suivante:

(26)

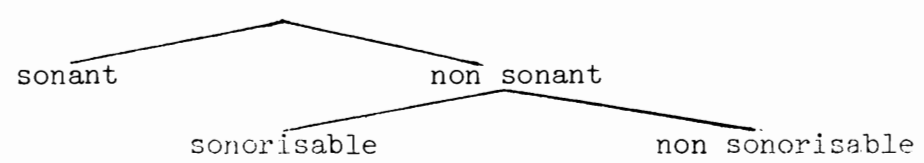

Il est possible d'établir l'ensemble des classes fonctionnelles du système; les difficultés ne vont surgir que lorsqu'il s'agira d'établir la hiérarchie des traits utiles à leur définition. Nour aurons les classes suivantes pour la catégorie [sonant]:

$\begin{array}{ll}\text { [labial, oral] } & \mathrm{mb}, \mathrm{b} \\ \text { [labial, non oral] } & \mathrm{m} \\ \text { [non labial, non oral] } & \mathrm{n}, \mathrm{n}, \mathrm{D} \\ \text { [non labial, oral, liquide] } & \mathrm{r}, \mathrm{I} \\ \text { [non labial, oral, non liquide] } & w, y\end{array}$

Chacune de ces classes fonctionne de façon homogène, mais l'organisation hiérarchique des traits reste encore à définir. Si on se décide à choisir comme prochaine étape de la division la dimension [oral], nous aurons le regroupement suivant:

\section{(28) [oral] [non oral]}

$$
r, I, w, y, b, m b \quad m, n, r, n
$$

La dimension [nasal] ne peut pas être utilisée car son utilisation conduirait 
à introduire /mb/ dans la classe des nasales, en le séparant de /b/, ce qui serait maladroit, compte tenu de l'existence de la neutralisation entre $b \sim m b$ qui implique un rapport plus étroit entre /b/ et $/ \mathrm{mb} /$ qu'entre /m/ et /mb/. Ce choix nous conduit à isoler $/ \mathrm{m} /$ des autres consonnes labiales pour le regrouper avec les consonnes nasales; or nous avons vu également que /m/ et /b/ fonctionnent exactement de la même manière et que les nasales subissent des contraintes qui ne concernent pas $/ \mathrm{m} /$.

En fonction de celà nous considérerons que cette division n'est pas très heureuse; il convient d'établir une division qui rapproche $m$ et $b, \mathrm{mb}$, ce que nous pouvions prévoir. La solution la plus cohérente nous semble être fournie par l'utilisation directe de la dimension [labial]:

(29)

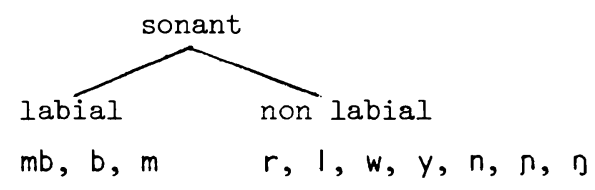

La division suivante s'effectue soit sur la dimension [oral] soit sur la dimension [nasal]. La dimension qui sera choisie en premier aura un statut plus général que l'autre. Nous savons qu'il faut décrire une neutralisation dont le terme marqué est nasal $m b \sim b$ en initiale et /b/ en finale); la dimension [nasal] peut, de ce fait, être considérée comme plus spécifique, et c'est donc la dimension [oral] que nous devrons choisir ici,

(30)

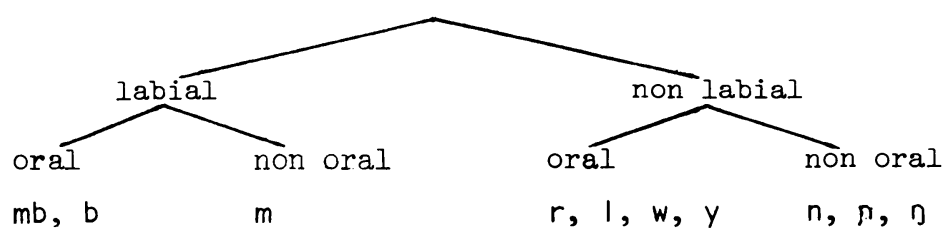

Il nous faut encore établir la division entre [liquice] et [non liquide] qui sert à isoler les semi-voyelles,

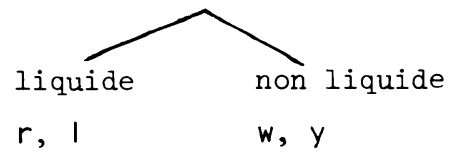


et nous avons achevé la structuration du système en corrélations.

En résumé:

(32)

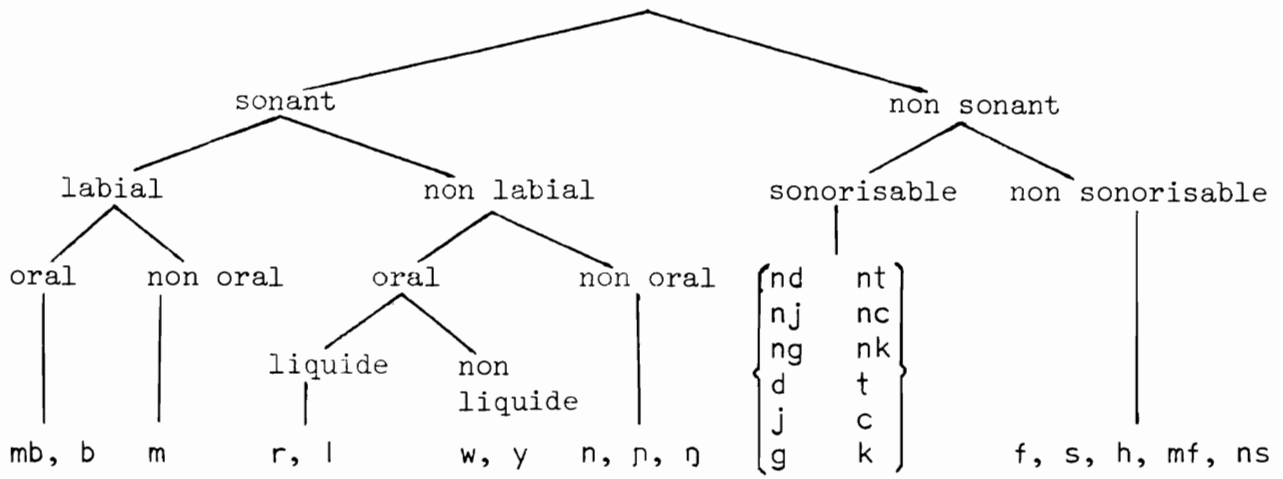

La division doit se poursuivre sur les traits de série. La corrélation [non sonant sonorisante] pose encore un problème de choix, vu qu'elle est déterminée à la fois par la dimension [nasal] et [sonore]. Il y a lieu de remarquer qu'il n'existe pas de neutralisation de sonorité alors que la neutralisation sur la dimension de nasalité est établie; la division se fera dans l'ordre:

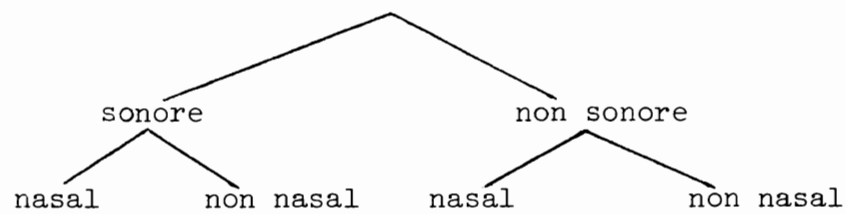

lequel donne un statut plus général à la dimension [sonore]. La dimension [nasal] sera utilisée de la même manière à l'intérieur de la corrélation $\mathrm{mb}, \mathrm{b}$ et de la corrélation $f, s, h, m f$, $n s$.

A leur tour les unités des corrélations liquide $r$, I et non liquide w, y seront établies selon les dimensions qui leurs sont propres. Il ne reste plus, à ce niveau, qu'a établir dans chacune des corrélations qui s'y prêtent la division en points d'articulations. Nous avons noté cidessus en $2.2^{9}$ qu'il était possible d'avoir une neutralisation entre des traits distinctifs dans une corrélation, à condition que la neutralisation

${ }^{9}$ Hypothèse liée à une conception hiérarchisée des faits structuraux, conception que nous présenterons dans un ouvrage à paraître "Les dialectes songhay". 
des traits de série ait déjà été établie. Ce cas se présente ici pour la catégorie $n, n, \eta$ qui n'est plus représentée en finale que par $n$. Nous nous servons de ce fait pour induire une première division sur la dimension [apical] qui nous donne le résultat suivant:

(34)

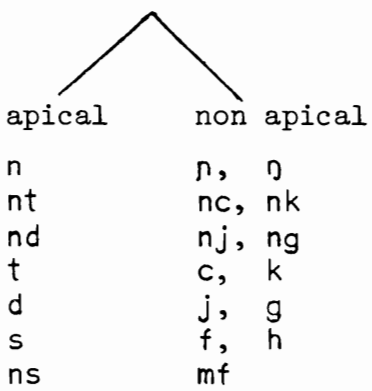

La dimension [apical] possède un certain nombre de caractéristiques du point de vue des combinaisons qui de toutes façons doivent lui permettre d'avoir un statut particulier; elle apparaitra comme la dimension nonmarquée au niveau des traits distinctifs et c'est par rapport à elle que s'établissent de nombreuses contraintes.

Ceci fait, la dimension [vélaire] permettra la division des sousclasses:

(35)
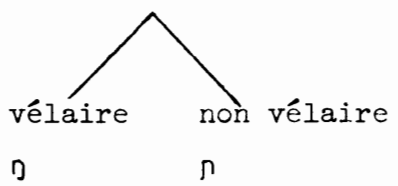

nk nc

$\mathrm{ng} \quad \mathrm{nj}$

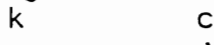

$\begin{array}{ll}g & j \\ h & f\end{array}$

5.2. L'incidence de $/ \mathrm{p} /$ et $/ \mathrm{mp} /$. Nous avons, dans cette analyse, éliminé complètement / $/$ et $/ \mathrm{mp} /$; cette élimination pas définitive, nous avons déjà traité ailleurs ${ }^{10}$ du cas de ces phonèmes et du problème que pose leur existence. Nous avions admis leur intégration et établi que cette même

$1{ }^{0}$ Nous renvoyons pour plus d'explication à l'article mentionné dans la note 4 . 
intégration avait une influence importante sur la structure de la langue en ce sens que celle-ci répondait alors à une double interprétation: l'existence de ces phonèmes implique le regroupement $\mathrm{de} / \mathrm{m} /$ avec les autres occlusives nasales et de /mb/ et /b/ avec les autres occlusives orales. Nous allons essayer de reprendre ce problème en tenant compte du schéma conceptuel ci-dessus proposé.

Le rapport se joue entre une corrélation de corrélation mb, b, $m$ et une pseudo corrélation $n d, d$, nt, $t, n \ldots$ Les deux caractérisées en substance (a) par le trait [occlusif] strictement redondant dans le système du point de vue fonctionnel, et (b) par une structure interne virtuellement identique. Le rapport $\mathrm{mb}, \mathrm{b}, \mathrm{m}$ actualisé dans le système est parallèle au rapport $n d, d, n$ non-actualisé.

I'interprétation de /p/ et / $\mathrm{mp} /$ implique un changement fonctionnel dans les traits de corrélation et une généralisation: si /p/ est intégré, alors /b/ devient [sonorisable]. Si le couple p, b devient [sonorisable], il glisse dans la corrélation définie par cette propriété; le même raisonnement peut être fait pour $\mathrm{mp}, \mathrm{mb}$. De ce fait, l'ancienne corrélation [labial] ne possède plus qu'une seule unité; le trait de corrélation et le trait distinctif déterminent alors la même unité. Dans ce cas, le trait [labial] peut devenir distinctif dans la corrélation [sonorisable] et $/ \mathrm{m} /$ se définira comme [sonant, labial et non-oral]. I] se regroupera avec la corrélation définie comme [sonant et non-oral] à l'intérieur de laquelle le trait [labial] aura une fonction distinctive parallèle à celle qui est devenue la sienne dans la corrélation [sonorisable], ce qui est une simplification puisque le même trait phonétique détermine une même fonction. En conclusion la corrélation [labial] disparaît.

Le seul changement réel a été le changement de statut de la dimension [labial], déterminé par l'incompatibilité entre [sonorisable] et [sonant] qui a une fonction catégorielle, puisqu'il définit la position postvocalique dans sa totalité. 


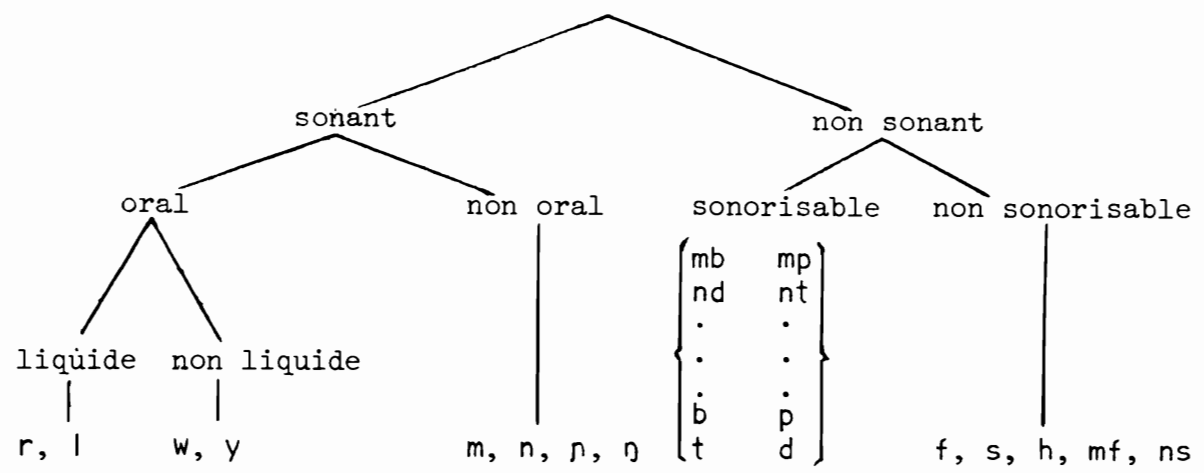

Ce changement est le résultat de l'action de deux processus:

(a) Influence de la substance phonétique sur la structure abstraite de la langue: indépendamment de toute considération fonctionnelle, dans l'établissement d'un rapport pseudo-corrélatif, à partir des unités phonématiques réalisées nous pouvions entrevoir l'existence latente des unités $/ \mathrm{p} /$ et $/ \mathrm{mp} /$. L'intégration de ces unités, d'abord refusée par la structure, a finalement eu lieu moyennant une restructuration.

(b) Influence du paradigme sur le syntagme: une pseudo-corrélation est la description d'un rapport paradigmatique pur au niveau de la phonétique distinctive. Le regroupement des labiales, auquel nous opérons dans la pseudo-corrélation, traduit l'effet de la contrainte exercée par ce rapport paradigmatique sur la structure syntagmatique. Il est remarquable que la structure syntagmatique réagisse sur les données en faisant disparaitre / $b$ / de la position postvocalique $(b \rightarrow m, b \rightarrow w)$, ce que montrent de nombreuses variations actuelles. Cette réaction peut être présentée comme un élément de preuve de l'intégration de /b/ et /m/ en face des autres occlusives: l'unité intégrée tend à fonctionner comme les autres unités de l'ensemble du point de vue distributionnel.

Ici se dégage un jeu entre le paradigme dont la description s'établit à partir de la substance phonétique et le syntagme dont la description s'établit à partir d'un espace organisationnel: ce jeu définit un champ interprétatif. Il convient sans doute de rapprocher cette présentation des conceptualisations proposées par Martinet [1955] dans se travaux sur l'attraction des systèmes, le renforcement des unités corrélatives, les problèmes de stabilité, etc. 
En conclusion, nous dirons qu'au point de vue pratique il se dégage, dans le songhay de Tombouctou, une prédominance de l'organisation paradigmatique sur l'organisation syntagmatique qui a pour conséquence de régulariser la structure à partir de l'organisation de l'espace phonétique. Nous allons étudier un autre point illustrant cette tendance.

5.3. La dimension nasale. La dimension nasale avait une fonction complexe en songhay. Si l'on prend le cas du zarma, la nasalité était utilisée en combinaison avec:

(1) les traits [sonant] et [consonantique] pour établir une corrélation de nasale, fonctionnant en position post et prévocalique: $\mathrm{m}, \mathrm{n}, \mathrm{n}, \mathrm{n}$;

(2) en combinaison avec le trait vocalique pour établir une corrélation d'unités syllabiques: i, ẽ, ã, õ, ̃ .11

A Tombouctou nous remarquons une évolution dans l'utilisation de la dimension en question. La corrélation nasale définie en ( 1 ), $m, n, n, n$ existe toujours. Seulement, la neutralisation dans ce cadre est beaucoup plus avancée: tandis que l'on trouve $m, n, D$ à Dosso, on ne trouve plus que $m, n$ à Tombouctou. Nous avons vu d'autre part que notre interprétation des occlusives nasales post-vocaliques à l'intérieur du mot nous permettait d'établir un paradigme de consonnes prénasalisées. La différence est donc que la corrélation des nasales disparaît de la position post-vocalique et se renforce en position prévocalique. La caractérisation de ces deux positions s'établit sur la dimension [sonant] et les nasales tendent à se "désonantiser". Il existe en zarma un paradigme d'unités syllabiques nasales qui comprend très peu d'unités, mais qui sont très courantes. La comparaison entre ce que l'on trouve à Dosso et ce que l'on trouve à Tombouctou est intéressante:

${ }^{11}$ Le phénomène des nasales syllabiques a été traité sur le cas zarma dans Nicolaï [1976]. 


$\begin{array}{lll}\text { a. hẽ } & \text { 'pleurer' } & \text { hem } \\ \text { hã } & \text { 'demander' } \\ \text { hi } & \text { 'tiens' } & - \\ \text { hõ } & \begin{array}{l}\text { 'aujourd'hui' } \\ \text { superlatif de } \\ \text { noir (< Bambara?) }\end{array} & \text { fi } \\ \text { b. nkor 'os de la hanche' } & \text { nkor } \\ & \text { nduna 'monde' } & \text { nduna }\end{array}$

Dans le cadre de (b) l'interprétation fournie à Tombouctou des séquences [\# $n+C[$ _ conduit à refuser l'existence d'une nasale syllabique et à accepter des consonnes prénasalisées. La conséquence

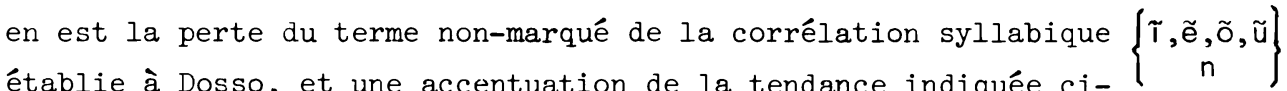
dessus puisque l'on a $\mathrm{n}+\mathrm{C} \rightarrow \mathrm{nC}$, soit une consonification de l'unité syllabique.

Dans le cadre de (a) nous relevons également une tendance à la consonification qui s'illustre par la dénasalisation de hẽ $\rightarrow$ hem .

L'existence de l'unité $[\mathrm{fT}]$ pose un nouveau problème en ce sens qu'elle semble impliquer la disparition des nasales en tant qu'unités syllabiques pour les rétablir sur le plan phonématique comme phonèmes vocaliques puisque nous aurions l'opposition entre / $f /$ et $/ \mathrm{h} /$ devant une voyelle nasale.

Le parler de Tombouctou posséderait ainsi un paradigme réduit de voyelles nasales. Toutefois, les attestations $\tau$, ẽ, õ que nous relevons pourraient être ici interprétées comme des séquences $\mathrm{V}+\mathrm{D}$ ce qui, d'une part recréerait en position finale le paradigme $m, n$, $j$ qui semblait réduit à $m, n$, d'autre part supprimerait la notion de nasales syllabiques. Cette interprétation supprime complètement ce phénomène "syllabique" sans créer un nouveau paradigme de voyelles nasales. Le passage des syllabes nasales à des combinaisons $V+\eta$ est aussi un effet de la "désonantisation" que nous soulignions, car une nasale syllabique est plus sonore qu'une occlusive nasale post-vocalique. Nous remarquerons, tout au long de cette description, une démarche généralisatrice évidente 
qui conduit finalement à l'unification, dans tout le système, d'un rapport corrélatif latent rapprochant le schéma structural du parler de l'organisation optimale de sa structure phonématique.

D'autres généralisations simplificatrices peuvent être étudiées dans le système morphologique du parler. Peut-être est-il intéressant de noter, en regard de ces faits, la fonction particulière de ce parler qui est un véhiculaire dans sa région.

6. Remarques Comparatives: Etude des Variations Tombouctou - Jennê Ngorku

Nous présentons ci-dessous les différences essentielles que nous avons relevées entre le parler tombouctien, celui de Jenné et celui de Ngorku. Ainsi que nous l'avons indiqué au début, il ne s'agit que de remarques différentielles; la substance même de ces différences ne nécessite pas un travail monographique séparé. C'est donc à partir d'une série de tableaux et de remarques que nous allons essayer de cerner la spécificité des variantes en question.

6.1. Remarque sur d'hypothètiques nasales vélaires en position postvocalique. Il semblerait que l'on puisse trouver [ $\mathrm{g}]$ en position finale à Jenné et Ngorku. Seulement il apparaît d'une manière anarchique et en particulier il n'apparait pas pour les mots qui ont effectivement ce phonème à cette place en zarma. Nous avons relevé les examples dans (38):

\begin{tabular}{|c|c|c|c|c|}
\hline & Tombouctou & Jenné & Iggorku & Zarma \\
\hline 'rat & ncom & ncam & DCEm & čan \\
\hline 'tomber' & kam & kam & kam & kân \\
\hline 'mettre' & dam & dam & & dèn \\
\hline 'manquer' & jen & jen & jen & jân \\
\hline \multicolumn{5}{|l|}{ mais } \\
\hline 'mûr' & nin & nin & ni $\left\{\begin{array}{l}0 \\
n\end{array}\right\}$ & niń \\
\hline 'traverser' & $d \varepsilon n$ & $d \varepsilon \eta$ & $\mathrm{d} \varepsilon \mathrm{m}$ & \\
\hline 'four' & al foron & alforon & alforon & \\
\hline 'guêpe maçonne' & b imb im & binbin & binbin & bínbini \\
\hline 'fromager' & bantan & bonton & bantam & bàntán \\
\hline 'chaleur' & koron & koron & $\operatorname{kor}\left\{\begin{array}{l}n \\
n\end{array}\right\}$ & \\
\hline 'amer' & horon & horon & & \\
\hline
\end{tabular}


(38) cont.

$\begin{array}{lllll} & \text { Tombouctou } & \text { Jenné } & \text { Ngorku } & \text { Zarma } \\ \text { 'entendre' } & \text { mom } & \text { mon } & \text { mom } & \\ \text { 'faire' } & \text { dam } & \text { dan } & \text { dam } & \\ \text { 'conduire' } & \text { hun } & \text { hum } & \text { hũnu } & \text { fûn } \\ \text { 'électricité' } & \text { ku:ran } & \text { kuran } & \text { kuran } & \text { kù:rân } \\ \text { 'menton' } & & \text { dankan } & \text { danka } & \\ \text { 'capok' } & & \text { bumbun } & & \end{array}$

Nous pensons que les réalisations notées [ $[0]$ sont en fait des réalisations de $/ \mathrm{n} /$, auquel cas il y a la même neutralisation à Jenné et à Ngorku qu'à Tombouctou, ce qui nous semble le plus probable. A la limite, si la réalisation [ $[\mathrm{J}$ représente des réalisations relachées de $/ \mathrm{n} /$, cela est conforme à une utilisation de l'espace phonique dans la mesure où $/ \mathrm{n} /$ en position finale représente l'archiphonème de la corrélation, lequel doit se différencier au maximum du phonème $/ \mathrm{m} /$. Il ne faut cependant pas oublier que les exemples présentés sont minoritaires et ne présentent aucune régularité; la plupart des autres attestations présentent des faits parallèles aux faits tombouctiens.

6.2. Remarque sur les variations nasales $\rightarrow$ orales: $b \leftrightarrow w \leftrightarrow b \leftrightarrow m$. Attestations:

\begin{tabular}{|c|c|c|c|c|}
\hline 'léger' & $\begin{array}{l}\text { Tombouctou } \\
\text { felow }\end{array}$ & $\begin{array}{l}\text { Jenné } \\
\text { felem }\end{array}$ & $\begin{array}{l}\text { Ngorku } \\
\text { felem }\end{array}$ & Zarma \\
\hline 'aveugle' & danow & danam & danam & dànòw \\
\hline 'politesse' & ladab & Iadam & ladam & làlábù \\
\hline 'froisser' & tutub & tutum & tut um & tút úbú \\
\hline 'respecter' & jejहw & je jow & jejem & \\
\hline $\begin{array}{l}\text { 'séparer le } \\
\text { mil du son' }\end{array}$ & sasow & sosow & sosom & sásábú \\
\hline 'heurter' & ceb & cow & cहm & \\
\hline 'carder le fil' & $I \varepsilon w$ & low & $\operatorname{l\varepsilon m}$ & \\
\hline 'nécessité' & (al)wa: jib & (al)wa: jum & (a) $\{w\}$ a: jum & àl wá: zíbì \\
\hline $\begin{array}{l}\text { 'cracher } \\
\text { avec bruit' }\end{array}$ & albalam & albalam & albalaw & \\
\hline 'élanger' & som & sow & som & \\
\hline loum' & kongom & kongow & & kàngòw \\
\hline
\end{tabular}


Ces variations sont réelles. En ce qui concerne $/ \mathrm{w} /$ et $/ \mathrm{m} /$, il ne se dégage aucun caractère de régularité; il semble s'agir de changements isolés et il semble que ni les possibilités distinctives dans les paradigmes concernés, ni la définition des phonèmes ne soient affectés par ces variations.

Nous trouvons à Jenné comme à Ngorku l'opposition $m \sim w$ en finale.

Attestations:

(40) Jenné-Igorku

$-w \#$

'enlever'

'carie'

'vent'

'Iire' kow

gow

hew

cow
Jenné-Ngorku

$-m$ \#

'arracher' kam

'avaler' gom

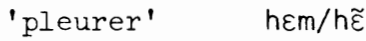

'forgeron' jहm

'rat'

ncam/ncem

$-n \#$

'construire' cen

'jour' han

'où'

men

'manquer' jहn

Par contre les attestations qui comportent /b/ post-vocalique à Tombouctou, ne sont généralement pas réalisées avec ce phonème à Jenné et Ngorku, surtout en position finale. Exemples:

$\begin{array}{llll} & \text { Tombouctou } & \text { Jenné } & \text { Ngorku } \\ \text { 'calculer' } & \text { haseb } & \text { hasaw } & \text { hisaw } \\ \text { 'force quelqu'un; wa:zib } & \text { wa:jum } & \text { wa:jum } \\ \text { nécessité' } & & \\ \text { 'politesse' } & \text { alwahab } & \text { alwahaw(b) } & \\ \text { 'froisser' } & \text { tutub } & \text { tutu/tutum } & \text { tutum } \\ \text { 'politesse' } & \text { ladab } & \text { ladam } & \text { ladam } \\ \text { 'donnerdescoups' jeb/jew } & \text { jew } & \\ \text { 'articulation' } & \text { dob/dow } & \text { dow } & \text { don } \\ \text { 'livre } & \text { kitab/kitaw } & \text { kitaw } & \text { kita:bu } \\ \text { 'plante remède' } & \text { sewsab } & \text { samsam } & \text { samsam }\end{array}$


(41) cont.

\begin{tabular}{|c|c|c|c|}
\hline & Tombouctou & Jenné & Ngorku \\
\hline 'écraser' & $\operatorname{titib}(w)$ & tut um & \\
\hline 'intercession' & assaba & assabow & assabow \\
\hline 'heurter' & $c \varepsilon b$ & cow & $c \varepsilon m$ \\
\hline 'samedi' & assawdu & al sawdu & $\operatorname{assab}(w) d u$ \\
\hline 'encens' & jibda & ( jibda) & \\
\hline 'broderie' & sibka & siw(b)ka & sibka \\
\hline 'citrouille' & IEbtanda & lawtanda & lawtanda \\
\hline $\begin{array}{l}\text { 'morceau de } \\
\text { calebasse' }\end{array}$ & cemsu & cebsu/kewsu & kemsu \\
\hline 'pied d'animal' & kow $\int i$ & kow $\int i$ & komsi \\
\hline 'Tabaski' & cibsi & $\operatorname{ciw}(b) s i$ & cimsi \\
\hline 'navet' & libt $i$ & & \\
\hline
\end{tabular}

Les parlers occidentaux de Jenné et Ngorku se caractérisent, par rapport à Tombouctou, par le fait que l'opposition $b \sim m$ et $b \sim w$ est perdue en position finale absolue et qu'elle reste à l'état résiduel avec en général des variantes libres en position postvocalique suivie par une autre syllabe.

6.3. Remarque sur la variation phonetique $w / v$. Les dialectes occidentaux de Jenné et Ngorku possèdent des réalisations fricatives labio-dentales du phonème /w/, à savoir [v] ou [vw], qui sont illustrées par le tableau suivant:

$\begin{array}{ll} & \text { Tombouctou } \\ \text { 'être malade' } & \text { wirci } \\ \text { 'lire le Coran' } & \text { wirdi } \\ \text { 'concession' } & \\ \text { 'soir' } & \text { wi } \\ \text { 'tuer' } & \text { wir } \\ \text { 'chercher' } & \text { wore } \\ \text { 'machoire, joue' } & \text { wannasu } \\ \text { 'courtiser' } & \text { wanga wanga } \\ \text { 'se promener' } & \text { warra } \\ \text { 'lancer' } & \end{array}$

$\begin{array}{ll}\text { Jenné } & \text { Ngorku } \\ \text { wirci/virci } & \text { virci } \\ \text { virdi } & \text { virdi } \\ \text { vindi } & \text { vindi } \\ \text { vikir/wicir } & \text { vicir } \\ \text { vi } & \text { vi } \\ \text { vir/wir } & \\ \text { were } & \text { vere } \\ \text { wannasu } & \text { vwannasu } \\ \text { wanga } & \text { vwanga vwanga } \\ \text { warra } & \text { warra/vwarra }\end{array}$


(42) cont.

\begin{tabular}{|c|c|c|c|}
\hline & Tombouctou & Jenné & Ngorku \\
\hline 'prêcher' & wa: ju & wa: ju & $v_{w} a: j u$ \\
\hline 'gros' & woro & worow & $v_{\text {woro/woro }}$ \\
\hline 'refuser' & wongu & wangu & wongo \\
\hline 'juste' & a sawa & a sawa & a sava \\
\hline 'avoir le temps' & & $\begin{array}{l}\text { alw }(v) \operatorname{ax}(k, q)- \\
\text { ati }\end{array}$ & alvakat $i$ \\
\hline 'moyen' & sasawante & isawante & isavant \\
\hline 'large' & & iruwa/i I wa & yilva \\
\hline 'veau' & ya:w & hawi : je & havi: je \\
\hline 'fiancaille' & & & viruwe \\
\hline
\end{tabular}

Nous avons un conditionnement en variantes; tandis que /w/ est toujours réalisé [w] à Tombouctou, il est réalisé

$$
\begin{aligned}
& \text { (43) a. } \left./ w / \rightarrow[v] /[]_{[}\right] \text {à Jenné } \\
& {\left[\begin{array}{ll}
+ & \text { ant } \\
+ & \text { fer }
\end{array}\right]} \\
& \text { b. } / w / \rightarrow[v] /[[i, e, a] \text { à Ngorku } \\
& \text { [- post] }
\end{aligned}
$$

En fait, à Ngorku la situation est renversée en ce sens que /w/ a plus souvent la réalisation $[v]$ que $[w]$.

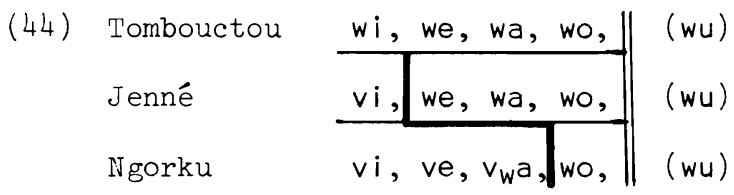

Un petit problème peut être soulevé: avons nous affaire à un phonème /w/ réalisé [V] ou bien un phonème / $/$ / réalisé [w]? Dans le premier cas nous sommes en présence de l'ensemble: $w \sim y$, dans le deuxième cas nous avons l'opposition $f \sim v$.

6.5. Remarque sur la variation phonetique $s / \int$. Nous relevons un certain nombre de palatalisations de /s/ devant/i/ aussi bien à Tombouctou qu'à Jenné, mais aucune palatalisation n'apparaît à Ngorku où le /s/ devant /i/ est réalisé avec toute la netteté souhaitable. Exemples: 
(45)

'viande'
'écraser'
'fonio'
'chien'
'estomac'
' 'ugement'
'trapper avec
une cravache'
'cure-dent'
'pied d'animal'
'première chambre'

Tombouctou

ba $\int \mathrm{i}$

gassi/garsi

gen $i$

$\operatorname{han} \int i$

$k u: \int e$

a a ara

sa:tu/ $a: t u$

$k 0 \int i$

kow $i$

almu war
Jenné

ba $\mathrm{i}$

garsi/gar $j i$

gen $\int i$

hansi/han $\int i$

$\mathrm{ku}: \int \mathrm{e}$

assara

so:te

kosi

kow $\mathrm{i}$

almuswar

(véranda)
Ngorku

basi

gars $i$

jensi

(reste du mil tamisé)

hans $i$

$\mathrm{ku}: \mathrm{s} \varepsilon$

assara

kos $i$

komsi

A Tombouctou, les faits peuvent, dans leur plus grande partie, être prévus à partir de la règle de variante suivante: $/ s+i / \rightarrow\left[\int+i\right]$ en position intervocalique, quand il est précédé par une consonne ou par une voyelle différente de /i/. Cependant, cette règle ne rend pas compte

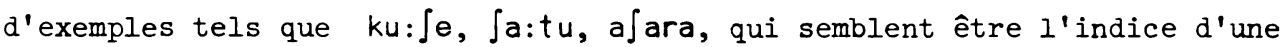
phonologisation des variantes $\left[\int\right]$ de $/ \mathrm{s} /$. A Jenné la règle est beaucoup moins stable, les variantes avec $\left[\int\right]$ sont semble-t-il beaucoup plus rares. A Ngorku il n'est jamais relevé de palatasation.

Il apparaît ainsi, à ce niveau, une différence-au moins entre les parlers de Ngorku et de Tombouctou-dans la mesure où les variations relevées à Jenné son suffisamment lâches pour ne pas pouvoir être formalisées par des règles combinatoires. Nous considérons que ce parler possède une tendance à la palatalisation $d u / s /$, tendance qui apparâ̂t dans la fréquence des variantes libres réalisées [ $\left.\int\right]$. Il faut aussi ne pas perdre de vue, comme cela s'est fait à Gao, comme cela est en train de se faire à Tombouctou, que l'affermissement de ce phénomène conduit aisément à la phonologisation des variantes.

6.5. Remarque sur les combinaisons $r+$ [apical]. Examples: 
$r+s$

'mépriser'

garsaka/gassaka

'fil' jerse/jesse

garsaka

garkasa

'écraser'

garsi/gassi

jesse/gerse

jerse

'disparaitre'

mursu/mussu

gar $j i$

garsi

'projeter de I'eau'

firsi

mursu

mursu

'démangeaison'

kursa

firsi

$r+t$

'déchirer'

kottu

kursa

kursa

'roter'

jettu

kortu

kottu

'délai'

satt $\mathrm{i}$

jettu

gertu

sart i

sart i

Ces variations sont dues à des dissimilaitons ou à des assimilations caractéristiques de certains parlers songhay. Ce ne sera qu'après une analyse globale que nous pourrons dire s'il s'agit d'assimilation ou de dissimilation. Nous ne pouvons que remarquer que les trois parlers ne se comportent pas de la même manière devant ces combinaisons, ce que nous avions déjà souligné à propos des combinaisons rs // ss. Les règles, dans la mesure où elles se dégagent, semblent tenir compte de la nature occlusive ou non occlusive de la dernière consonne de la combinaison.

6.6. Remarque sur la distribution du phoneme $/ \mathrm{s} /$. Ces parlers semblent accepter l'existence de / $/ \mathrm{s}$ en position postvocalique. Ce serait la seule attestation d'une consonne non-sonante dans cette position.

Exemples :

(47)

'véranda'

'se rincer la bouche'

'chapelet'

'gardien'

'serpent cracheur'

'mendiant'

'couscous'

'plaque de cheval'
Tombouctou Jenné

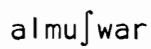

kukuJi

tasibaha

and

kuskusa

kasaji

kaske almuswa:r

kus(u)kus(u)

kuskus

tasibiya

koroskwey

fostere

almiski:n

kuskusu

kus $_{u}$ kusu

kasike

kesegi 
(34) cont.

$$
\begin{aligned}
& \text { Tombouctou Jenné Ngorku Dolbel } \\
& \text { 'décoration de porte' mismar } \\
& \text { 'chambre double' taska } \\
& \text { 'pointu' } \\
& \text { tasika } \text { tas }_{\mathbf{i}} k a \\
& \text { mesmesu }
\end{aligned}
$$

\section{BIBLIOGRAPHIE}

Dupuis-Yakouba, A. 1917. Essais de Méthode pratique pour l'étude de la langue songaï. Paris: E. Leroux.

Martinet, A. 1955. Economie des changement phonétiques. Berne: Francke S.A.

Prost, A. 1956. Le sonay et ses dialectes. Dakar: M. IFAN.

Tersis, N. 1972. Le zarma: étude du parler djerma de Dosso. Paris: SELAF.

Nicolaí, R. 1976. "Notes sur (et à partir de) la phonologie du zarma." Bulletin de I'IFAN 38:67-126.

Nicolai, R. 1977. "Réinterprétation et restructuration en zarmasonghay." Bulletin de I'IFAN.39 (B).1. 
FOCUS IN THE RENDILLE CLAUSE

Antoinette Oomen

In the following study an attempt is made to analyze focus structure in the Rendille Clause. Focus structure is described in Halliday's terminology [Halliday 1967, 1970 . In Rendille there are two focus markers, here called +predicate focus marker and -predicate focus marker, from now on + pfm and $-p f m$. In the information unit, most often the clause, a focus marker is obligatory; secondly there is only one focus marker allowed in the information unit, either a +pfm or a -pfm. The +pfm á, prefixed to the predicate, has, besides bringing into focus the predicate, a second function in that it realizes the unmarked focus, appropriate to the first information unit in a discourse. Taken out of its context, á is ambiguous. The -pfm é, suffixed to a noun or nounphrase in focus, appears to have developed from a relative clause construction, but is no longer considered such by the native speakers. Phonological, syntactic and semantic arguments are presented to support this hypothesis.

1. Introduction

The Rendille language is spoken by approximately 15,000 nomadic people who inhabit the southern part of Marsabit District in Northern Kenya. The Rendille rely on camel economy, supplemented by small stock. After several years of severe drought goats and sheep are nowadays valuable cash providers, which enable their owners to buy additional food stuffs like maize meal.

According to Heine [1976] Rendille belongs to the Sam group of Cushitic languages, which forms a sub family of the Afroasiatic (Erythraic) language family. The Sam languages which form the eastern sub-group of Omo-Tana, a sub-group of Lowland East Cushitic are subclassified in the following way [Heine 1976]:

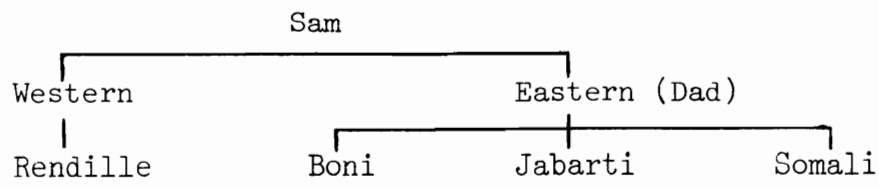


Previously published materials on Rendille consist only of some very brief notes by Fleming [1964] and a grammatical sketch by Heine [1975/ 76]. 1

The present article is based on a M.A. thesis submitted to the University of Nairobi in 1977 [Oomen 1977a]. ${ }^{2}$

In the introduction of the present article a few aspects of Rendille phonology and morphology will be presented, aspects that were previously unknown or incompletely given and that are relevant to the following discussion of focus structure. Since phonology falls outside the concern of this study, the phonemic inventory of Rendille, presented to serve as a key to the transcription used in the examples, is given without comment except for one phonological rule whose discovery facilitated the analysis of focus structure and related problems.

1.1. Phonemic inventory.

Consonants

Bil. Labio- Dent. Alv. Post- Pal. Vel. Phar. or dental alv. Glottal

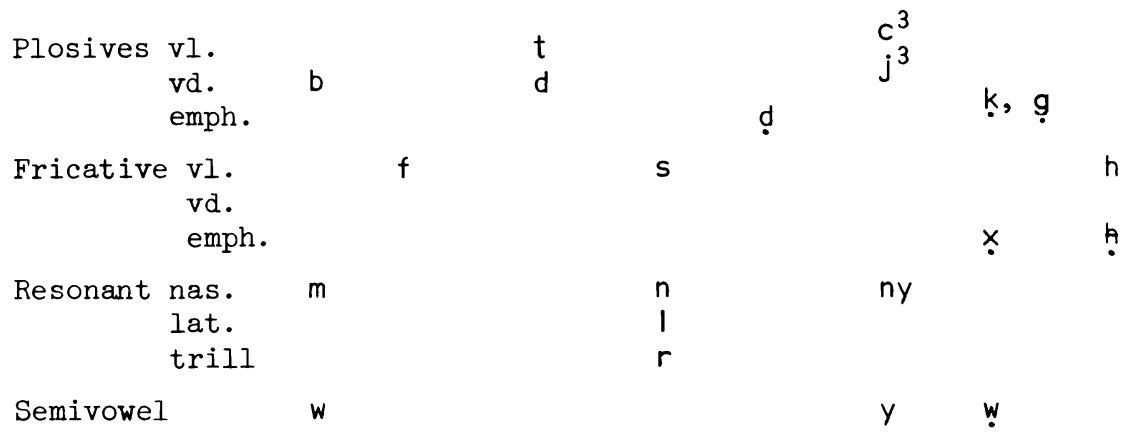

${ }^{1}$ I would like to acknowledge the encouraging help I received from Prof. Bernd Heine who supervised my M.A. thesis. I am also grateful to Dr. R. Hudson for stimulating discussions we had during his short visit to Nairobi.

${ }^{2}$ I am most grateful to Mr. Bernard Barchuma Arbele from Korr who was my principal informant.

${ }^{3}$ The affricates $\underline{t s}$ and $\underline{d z}$ seem to be common allophones of $\underline{c}$ and I. 
Vowels

\begin{tabular}{lll} 
High & i & \\
Mid & & ${ }^{u}$ \\
Low & \multicolumn{2}{c}{$a^{u}$}
\end{tabular}

length of vowels and consonants is important but is not considered here. Consonant phonemes with a dot underneath could be called "emphatic". since they appear to be similar to Arabic emphatic consonants in that they colour adjacent vowels. Their main characteristic seems to be pharyngeal stricture caused by retraction of the tongue root and a simultaneous raising of the larynx. The emphatic consonant phonemes may be described as having the feature -ATR [Stewart 1967], while the different vowel qualities which are encountered may be characterized by + or - ATR. The rule proposed to account for the different vowel qualities is as follows [Oomen 1977b]:

$$
\left[\begin{array}{l}
- \text { Cons } \\
+ \text { Voc }
\end{array}\right] \rightarrow[\alpha A T R] \quad\left\{\begin{array}{cc}
{\left[\begin{array}{l}
+ \text { Cons } \\
- \text { Voc } \\
\alpha A T R
\end{array}\right]} & - \\
- & {\left[\begin{array}{l}
+ \text { Cons } \\
- \text { Voc } \\
\alpha A T R
\end{array}\right]}
\end{array}\right\}
$$

In the following discussion vowel allophones caused by an adjacent -ATR consonant will not be indicated. In cases where the influence of an adjacent -ATR consonant has a neutralizing effect on the vowel of a grammatical morpheme, this will be indicated in a footnote.

1.2. Tone and stress. ${ }^{4}$ It is assumed here that tone plays an important role in Rendille. It appears that stress is a concomitant feature of high tone only. A syllable that has high tone + stress is here called "prominent". 5 A prominent syllable is also longer than a non-prominent one.

The two phenomena tone and stress must be distinguished, however,

${ }^{4}$ Further investigation on the question of tone and stress is being undertaken. The following remarks must be considered preliminary.

${ }^{5}$ See Tucker and Bryan [1966:499] for tone and stress in other Cushitic languages. 
since high tone appears to be fixed and lexically relevant, while stress although always a concomitant feature of high tone, may move from one high tone syllable to another high tone syllable, depending on syntactic environment. Examples (prominent syllable is underlined):
(1) Inàm
'boy'
inanki dèr 'the tall boy'
inam áyimì 'the boy has come'
inamé yimi 'the boy has come' (item in focus is in italics)
Tone is one of the means to indicate gender (masculine and feminine) and number (singular and plural). Examples:
(2) gender: nỵ̣ràx 'young male camel' wòr 'news' (masc) nyiráx 'young female camel' wór 'well' (fem)
(3) number: wáràb 'big male sheep' waráb 'big male sheep (pl)'

On the syntactic level it appears that relative clause markers and focus markers a.o. have in all cases high tone + stress, i.e. prominence which is thought to influence the pitch of the surrounding syllables. This influence is however not indicated in the present study. In the present paper tone will be indicated as follows:

High: 1) +stress:

Mid: unmarked

Low: -

\subsection{The sentence.}

1.3.1. Word order. The predominant word order of sentence constituents in Rendille is Subject - Object - Verb.
(4) maxábal inam ájáhè
elder boy has beaten
'the elder has beaten the boy'
inámè wel ádíxtè
'the girl has washed the child' girl child has washed

The indirect object precedes the direct object. 
(5) an maxábállè gólol ásícè I elders food have given

'I have given food to the elders'

Adverbs and adverbial phrases precede the verb.

(6)
us celé yimi
he yesterday came
'he came yesterday'
us Korré káyimi
'he came from Korr'

he Korr ${ }^{-}$from-came

Qualifiers follow the noun and are introduced by a gender sensitive particle k- (masc sing), h- (masc pl), or t- (fem sing and pl).6

(7) hanti wèn 'big woven container'

woven container big

gali dáxàn 'white camels' $\left(k \rightarrow \phi / 1 \_\right)$

camels white

albehi innokorr kágarre, áyimì 'the girls we saw in Korr have come' girls we Korr in saw have come

Note however the following possible constructions:
(8) bèy áficedà
'edge of lake'
lake its edge
kùl wórisa káyenna 'container we are talking about' container its news we are talking about

as variants of afí bèy and worí kùl respectively.

1.3.2. Permutation of word order. OSV order has also been observed.

In this case there is a pause after the fronted object.

(9) inám, maxábal ájáhè 'the elder has beaten the girl' (verbal concor $\vec{d}$ is with maxábal, i.e. masc)

It is assumed here that in (9) inám 'girl' is thematized by fronting. The preferred translation was found to be 'the girl has been beaten by the elder' and not 'the elder has beaten the girl'.

Passivization is a well known means of thematizing the object in English. In Rendille there are other means of thematizing the object if

${ }^{6}$ Each gender sensitive particle, further on in this study called "relative clause marker", has a set of allomorphs depending on preceding final consonant or vowel of the noun to which it is attached. See Oomen [1977a] for more details. 
the agent is not mentioned, namely by a verbal prefix la- .
(10) inám álájáhè
'the girl was beaten'

Instances were observed where the predicate is fronted. This appears to be possible only if no more than one sentence constituent other than the predicate is present. There is a pause after the fronted predicate and the latter must have the predicate focus marker.

$\begin{array}{ll}\text { áyélà, inàm } & \text { 'the boy is doing it' } \\ \text { as a variant of inam áyélà } & \\ \text { áyè à, sáhtà } & \text { 'he will do it tomorrow' }\end{array}$

but $c f$.

*yela, ínàm

* yela, sáhtà

It appears then that in Rendille permutation of word order is a means of thematizing very much the same as Halliday [1967] indicates for English.

(12) gàl, sigir migasané, sugubé tigis 'disease did not kill the camels disease did-not-kill thirst killed camels, thirst did'

(note that the conversation is about camels)

Consider in this context bèy áficedà as a variant of afí bèy, where bèy is fronted, presumably because bèy is the theme.

Permutation of word order is certainly a feature different from Focus, which we shall see below is an obligatory feature of the Rendille clause and involves morphological and tonal changes.

1.3.3. Case. ${ }^{7}$ Greenberg [1966:96] states as his Universal 4l: "If in a language the verb follows both the nominal subject and the nominal object as the dominant order, the language almost always has a case system." In Rendille the dominant order is SOV. The absolute form of the noun in both genders is the accusative.

${ }^{7}$ For comparison see Heine [1975/76] and Oomen [1977a]. The adnominal genitive case is not considered here. In a restricted number of cases the genitive case is overtly marked by $-t$ suffixed to the possessornoun. 
(13) us dágàł ásáè 'he has thrown the stone' (dágàt masc)

$\begin{array}{lll}\text { an dafár áxábà 'I have cloth' } & \text { (dafár fem) } \\ \text { cimbir ál'yégis } & \text { 'the bird was killed' } & \text { (cimbîr fem) }\end{array}$

The nominative is only marked in the feminine gender and only on the noun unaccompanied by qualifiers; qualifiers are unmarked for case. This marker -̀̀ is suffixed to subjects of both transitive and intransitive verbs. It therefore appears to be a surface subject marker.

(14) maxabálè gólol ásógátè

inámè intané kajirta 'the woman has bought food'

(maxabál 'woman')

'the girl is here' (inám 'girl')

The transitivity relations [Halliday 1967:38-81] or case relations [Fillmore 1970] of agent and goal are realized by a variety of means in Rendille:

-by word order

-by subject-predicate agreement of gender and number

-by nominal suffix in a restricted use

-by verbal prefix. Many other cases are also expressed in the last mentioned way. For example

(15) Source, ká-: inamKorrếkáyimi 'the boy came from Korr' Manner, i-: sagón iyimi? 'how did he come?'

1.4. The noun phrase.

1.4.1. Noun classes. ${ }^{8}$ A classification is proposed that is based on gender and concomitant tone pattern in the singular. In the majority of Rendille nouns there is polarity of gender and number. This classification can predict the type of plural formation that is correct. A considerable number of Rendille nouns, however, have only a singular form.

Class 1. Class I contains all the nouns which have masculine gender in the singular and show the tone pattern high-low, which tone pattern can be reduced or extended according to the number of syllables into:

OThe following information is not to be considered complete. Further investigation is needed. 
high-falling: ', high-low: “, mid-high-low: -’, and mid-mid-high-low: - For class 1 a sub-classification is made as follows:

Class la. Class la contains all masculine nouns (high-falling tone pattern) which are monosyllabic. These nouns do not have the usual polarity of gender and number, but are masculine in singular and plural. There are a number of nouns in this sub-class that have singular forms only.

\section{(17) jìd 'meat' jît 'road' kùl 'container' òr 'male camel' singular form only: gàl 'camels' hòl 'impala'}

Class $1 \mathrm{~b}$. Class $\mathrm{lb}$ contains the masculine nouns which have more than one syllable. They accordingly have high-low, mid-high-low or mid-midhigh-low tone pattern.

(18) inàm 'boy' áràm 'husband' dúlbè 'roofmat' yábàr 'string' maxábàl 'elder' xorojébsì 'very large snake' singular form only: álbè 'girls' túmày 'male sheep or goats that have just been castrated'

Class 2. Class 2 contains the nouns that have feminine gender in the singular and which show the tone pattern mid-high, which can be reduced or extended according to the number of syllables as follows:

mid-rising: ', mid-high: -', mid-mid-high: - -'.

For class 2 no sub classification is needed.

(19) abár 'mother' ilím 'tear' úl 'stick' mandán 'twin' lóh 'leg' dowohó 'jackal'

singular form only: iy 'sheep' ríy 'goats'

1.4.2. Plural formation. Class la members form their plural by reduplication. This reduplication has three characteristics.

(1) it does not affect the initial consonant

(2) the reduplicated syllable has prominence

(3) the vowel of the reduplicated syllable is always a

(20) jìt - jitát kùl - kulál òr - orár (cf. (17) above)

Class lb members have a plural suffix -è (after vowel -inyè is found, after consonant this consonant seems to be geminated). The syllable preceding è has prominence. 
(21) áràm - arámmè dúlbè - dulbínyè yábàr - yabárrè (cf. (18) above)

Class 2 members have a plural suffix -ó (after vowel -yó is found).

(22) abár - arbó úl - uló dowohó - dowohoyó (cf. (19) above)

Exceptions to these rules are nouns that change their tonal pattern to make their plural:

(23) dúfàn 'camel kept for slaughtering' pl. dufán kélèn 'big male goat' pl. kelét

and those nouns where natural sex seems to override grammatical gender:

(24) hélèm (and not expected helém) 'male sheep' helmó fólàs (and not expected folás) 'castrated camel' pl. folasó

1.4.3. Dependent categories. All qualifiers of the noun except the numeral ${ }^{9}$ are introduced by a gender sensitive relative clause marker, $k \underline{i}$ or kiye for masculine singular, $k \underline{i}{ }^{10}$ or híye for masculine plural, and $+\underline{i}$ or tíye for feminine singular or plural.

$k \underline{i} y e$, híye and tiye are presumably derived from $k \underline{i}+e$, $h \underline{i}+e$ and $+\underline{i}+e$ respectively, $\underline{-}$ e being a subjunctive form of the copula, found only in subordinate clauses.

$k \underline{i}$ and $k \underline{i} y e, h \underline{i}$ and $h \underline{i} y e, t \underline{i}$ and $+\underline{i} y e$ appear to be in free variation.

$\left.\begin{array}{l}\text { (25) minki gòb } \\ \text { minkiye gòb }\end{array}\right\}$ 'the house of the settlement' (min 'house (masc)')

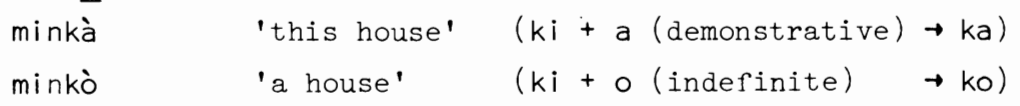

inántilye) xanan, ádéfsátè 'the girl who was ill has recovered' ( i nám 'girl $(\mathrm{fem})$ ')

Note however that the numeral is not introduced by a gender sensitive marker:

(26) Inam afàr 'four boys'

${ }^{9}$ Compare this situation with Somali, where the numeral precedes the noun. In Rendille tuman 'all' behaves in this respect like a cardinal number.

${ }^{10}$ Phonetically, hí is hé (see l.l). 
1.4.4. Adjective. A classification can be made, based upon the absence or presence of the copula yahe ( 3 pers sing) in the affirmative present (non-perfect) and upon the presence of either the negative marker -me (suffixed), which occurs also after nouns, or the negative marker ma(prefixed), which is a verbal negative marker. The copula is present in different forms in both classes in other than the unmarked present to serve as a mere locus for the marking of tense, mood and aspect. ${ }^{11}$ Class 1 will be examplified by bur 'big' (for persons: 'rather too big'). The noun used to illustrate corresponding behaviour will be mwalimu 'teacher'. Class 2 will be examplified by der 'tall'. The verb used to illustrate corresponding behaviour will be doon 'want'.

$$
\text { Adjective }
$$

Noun

$\begin{aligned} & \text { Class 1: aff. } \text { inam ábur } \\ & \text { 'the boy is big' } \\ & \text { neg. ' inam burmè } \\ & \text { 'the boy is not big' } \\ & \text { Adjective } \\ & \text { Class 2: aff. Inam áder yehe (are/A) } \\ & \text { 'the boy is tall' } \\ & \text { neg. ' inam máder } \\ & \text { 'the boy is not tall' }\end{aligned}$

inam ámwal imu

'the boy is a teacher'

inam mwal imumè

'the boy is not a teacher'

Verb

ínam ádoona

'the boy wants'

inam mádoono

'the boy does not want'

The numerals belong to class 1, which fact strengthens our hypothesis that the adjectives of class 1 are in fact nouns.

1.4.5. Personal pronoun. Personal pronouns and likewise pronominal adjectives and demonstratives have two paradigms. One could be characterized by the feature -FOCUS, the other by the feature +FOCUS. Examples will be given in the course of the discussion of the main part of this study on Focus. For complete paradigms, see Appendix I.

1.5. The verb. ${ }^{12}$ Rendille verbs are best arranged into two classes:

Class 1: prefix conjugation. Bound subject pronouns are prefixed to the verb stem. The bound subject pronouns for this class are:

${ }^{11}$ For more details see Oomen [1977a: 28].

${ }^{12}$ For more details see Oomen [1977a: 26-33]. 


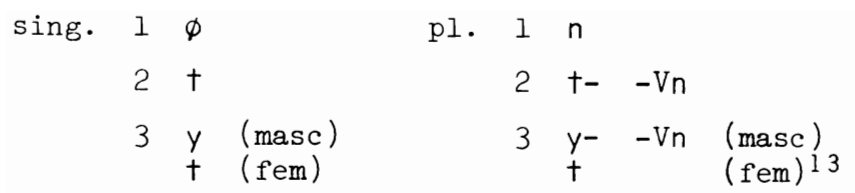

Prefix verbs form a minority of Rendille verbs (only thirteen have been found up until now). They belong to the basic vocabulary. Tense/mood distinctions are expressed by vowel alternations in the stem, namely $\mathrm{a} / \mathrm{i}$ and $\mathrm{a} / \mathrm{u}$. They sometimes have irregular and incomplete paradigms. In this class there seems to be no specific form for the subjunctive, the mood that is used in subordinate clauses. There are distinct optative forms. See Appendix I for a complete list of verbs found taking the prefix conjugation.

Class 2: Suffix conjugation. Bound subject pronouns are suffixed to the verb stem. There are no vowel alternations in the verb stem, the suffix vowel, however, changes according to tense/mood distinctions, namely a/e. This class contains the vast majority of Rendille verbs and is highly regular. The bound subject pronouns for this class are:

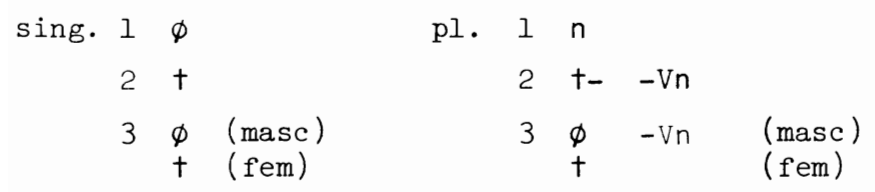

For class 2 a subclassification can be made into $2 \mathrm{a}$ and $2 \mathrm{~b}$, characterized among other things by different negative present tense suffixes, respectively -0 and $-i$. For class $2 b$ another subclassification can be made, characterized by a different form for the affirmative imperative, ending in respectively -0 and - so. Members of class 2 have distinct forms for the subjunctive, i.e. the same forms as for the negative present minus the prefixed negative marker ma- .

\section{Focus in the Rendille Clause}

2.1. Some general notes on focus structure. Focus structure is described in Halliday's terminology [Halliday 1967, 1970]. Halliday sees language in terms of its use. As act of speech is seen as a simultaneous selection

$13_{\text {The }} 3$ pers pl feminine has the same form as the 3 pers sing feminine. 
from among a large number of interrelated options. The system of available options is the grammar of the language. Any one clause is built up of a combination of structures deriving from the three functions of language to which correspond divisions in the grammar [Halliday 1970: 141-42].

2.1.1. Transitivity, mood, and theme. Halliday then regards the English clause as the domain of three main areas of syntactic choice [Halliday 1967:199]. These are:

- Transitivity: This is the set of options relating to cognitive content.

- Mood: This represents the organization of participants in speech situations.

- Theme: This is concerned with the information structure of the clause. 14

The present discussion will be mainly concerned with a set of options in the Theme structure of the clause, namely the information options. These involve discussing information units and information focus [Halliday $1967: 200-11] .15$

2.1.2. Information unit, clause, and information focus. According to Halliday any text in spoken English is organized into what may be called information units. A clause may contain two information units, it may be a single information unit, or it may itself be part of an information unit which includes other clauses. The unmarked option occurs when the

${ }^{14}$ Note that Halliday uses the term Theme also for a particular role in the distribution of information in the clause as a message, besides the notion just mentioned. I have briefly discussed some aspects of the Theme Rheme options in Rendille under 1.3.2.

15 Evidently Halliday's approach is based on English, but there are many parallels, which may indicate that his model can be applied to other languages than English. That information focus in English is realized by the use of phonological prominence, referred to by Halliday as "tonicity", but in Rendille by focus markers, which incidentally always have high tone + stress, does not affect the model; they are low-level language specific differences. 
clause contains one information unit. Note that a clause includes the clauses which are embedded within it.

It appears that in Rendille there is only one focal point possible in one information unit, the rest being non-focal (for exceptions to this statement, see 2.1.4. and (30)). The information focus falls on one constituent of the information unit. This one constituent which receives the information focus represents the new element in the message. 2.1.3. "Given" versus "new". We can say that the system of information focus assigns to the information unit (in our case the Rendille clause) a structure in terms of the two functions, "given" and "new". "Given" is presupposed knowledge by both speaker and hearer, "new" is non-derivable information. "New" is not only factually new, it may imply contrast with what has been said before. As is well known, given units are often represented anaphorically, by reference, substitution or ellipsis.

2.1.4. "Unmarked" versus "marked" focus. "Unmarked" focus is the form appropriate to the first information unit in a discourse. In other words, where the focus is unmarked, its domain may be the whole of the information unit, here the clause, i.e. the whole clause represents new information. The structure is not "given-new", but simply "new". Where the focus is marked, however, it may assign prominence to any constituent of the clause, the remaining being non-focal: there is a "given-new" structure.

2.2. Focus markers in Rendille. Rendille appears to have two focus markers.

l) + predicate focus marker (from now on called $+p f m$ ), realized by áprefixed to the predicate. ${ }^{16}$

16 For comparison see Bell [1953:25], Hetzron [1965:118] and others on Somali indicators waa and baa, which appear to have a function similar to Rendille á and é . An apparent correspondence ba was found in Rendille. Examples: yele túmamba átimi 'all the boys came' (túmàn 'all'); ébà 'nobody' (et (man) + ba), mélba 'nowhere' (mél $(p l a c e)+b a)$. Contrarily to $-p f m$ é, ba can occur simultaneously with + pfm á and also on nouns in isolation, i.e. without a predicate. 
(28) inam áyimi 'the boy came'

diri ákulel 'the pot is hot'

2) - predicate focus marker (from now on called -pfm), realized by -é suffixed to any other constituent of the clause, but to not more than one in the information unit, i.e. in the unmarked case the clause.

(29) inamé yimi

maxábàl inamé jahe

maxábàl intusé kajira

hanuki burenyé kajibte 'she put a lot of milk'

It seems that in Rendille the +pfm has a second function, namely it realises the unmarked focus appropriate to the first information unit in a discourse. 17

(30) inam áyimi

'the boy came', is a felicitous answer to the question: What happened? There is no given component in this message. The message contains all new information.

inamé yimi 'the boy came', however, is not a correct answer to: What happened? It is the correct answer to: Who came? or: Did the girl come?

inam áyimi 'the boy came', is as well the correct answer to: What did the boy do? or: Did the boy go away?

Taken out of its context, a sentence like inam áyimi is ambiguous.

2.3. Distribution of focus markers. In the Rendille clause a focus marker is obligatory, except for those cases where the information unit consists of more than one clause. Secondly, in the Rendille clause there is only one focus marker at a time, either a + pfm or a $-\mathrm{pfm}$.
(31) inam áyimì
*inam yimi ${ }^{18}$
'the boy came'

(no focus marker)

${ }^{17}$ Compare with a.o. Givon [1975:185]: "It is normally assumed for the 'neutral' sentence pattern of a language, that the predicate phrase contains the new information or asserted portion, while the subject phrase contains the new information or asserted portion, while the subject is the topic or presupposed portion with respect to which the assertion is being made."

${ }^{18}$ Starred forms indicate ungrammatical forms. 
(31) cont.

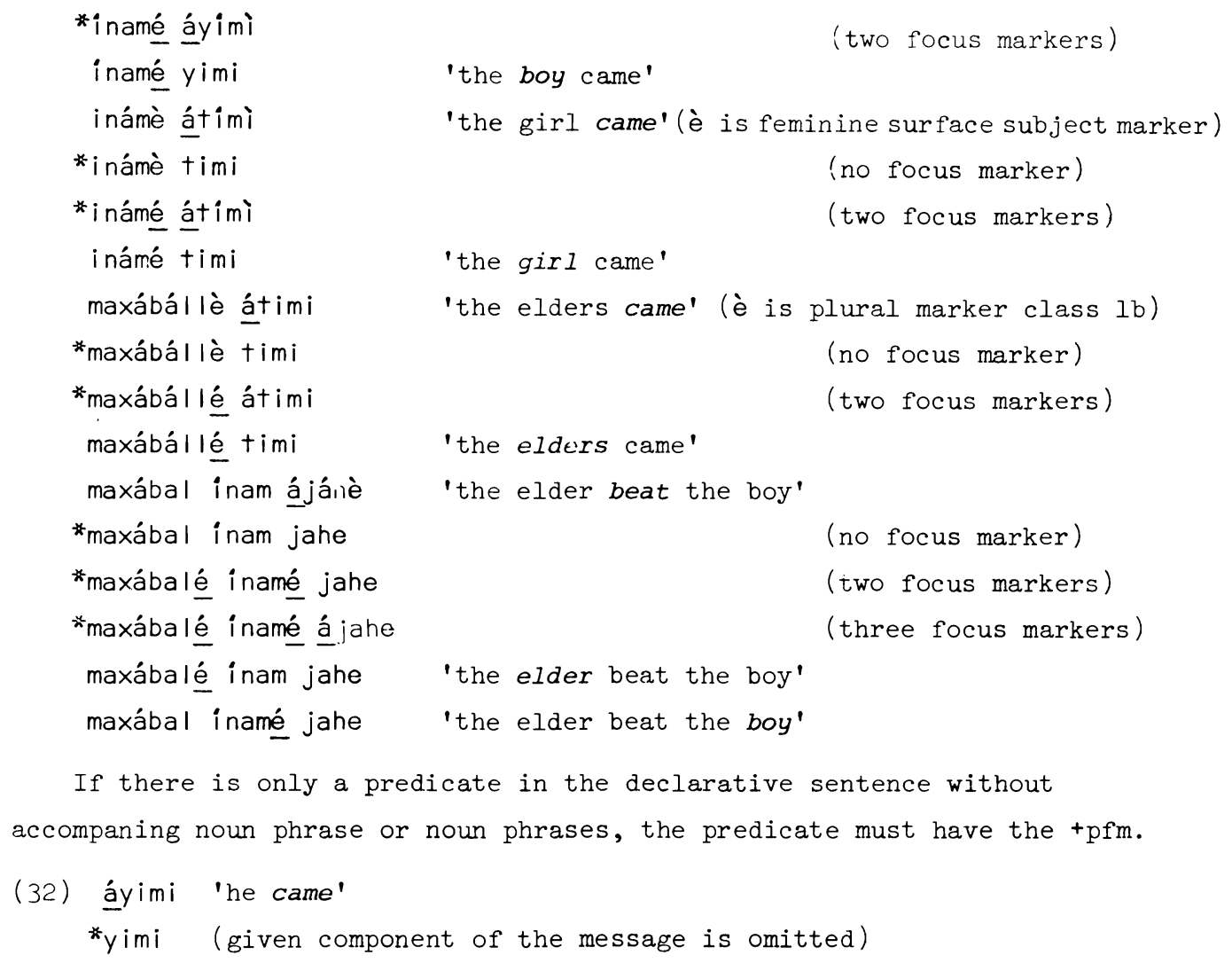

This is predictable, since áyimi contains the new information and so has focus.

The -pfm must precede the predicate. As the predicate normally is in sentence final position, this implies that a noun or noun phrase without an accompaning predicate, i.e. where the predicate is omitted because it contains the given component of the message, cannot have the focus marker. It however has focus.

(33) ayó yimi 'Who came?' can have two possible answers: inamé yimi 'the boy came'

*inamé


Note that the predicate is not always in sentence final position. This is the case when the predicate is fronted for Thematization. 19

(34) áyiroi, inam 'he has come, the boy'

It is assumed that in this case we have two information units (note pause after áyimi) each with its focus. Áyimi has the $+p f m$, while Inam by itself has focus but this focus is not expressed by a -pfm (see above). Here we find an interaction of the Theme and Information options.

2.4. Realizations of the focus markers.

2.4.1. Realizations of the +pfm. If the + pfm is prefixed directly to a verb stem beginning with a vowel, the á is elided but its high tone + prominence remains on the initial vowel of the verb stem.

$\begin{array}{lll}\text { (35) álbe írtè 'the girls went' } & \text { (ird 'go') } \\ \text { an úrdà 'I am sleeping' } & \text { (urd 'sleep') }\end{array}$

For the sake of clarity the examples will be restricted to simple declarative affirmative sentences containing only a subject and a predicate. Question and negation will be dealt with below.

\begin{tabular}{|c|c|c|c|}
\hline $\begin{array}{l}\text { verbal pr. } \\
\text { Class } 1\end{array}$ & $\begin{array}{l}\text { focus on predicate } \\
\text { inam áyámìt }\end{array}$ & $\begin{array}{l}\text { focus on subject } \\
\text { inamé yamit }\end{array}$ & 'the boy comes' \\
\hline $\begin{array}{l}\text { verbal pr. } \\
\text { Class } 2\end{array}$ & $\begin{array}{l}\text { an ággírdámà } \\
\text { gogág ánúhàn } \\
\text { komboró ájébìn }\end{array}$ & $\begin{array}{l}\text { aní girdama } \\
\text { gogágé nuha } 20 \\
\text { kombóró jebe } 20\end{array}$ & $\begin{array}{l}\text { 'I play' } \\
\text { 'the cameliskins smell' } \\
\text { 'the stools broke' }\end{array}$ \\
\hline $\begin{array}{l}\text { nominal pr. } \\
\text { with noun }\end{array}$ & $\mathrm{Nal}$ i yon ámwal imù & $\begin{array}{l}\text { Nal iyoné mwal ime } \\
(u+e \text { copula }) \rightarrow\end{array}$ & $\begin{array}{l}\text { 'Naliyon is a teacher' } \\
\text { e) }\end{array}$ \\
\hline $\begin{array}{l}\text { nominal pr. } \\
\text { with class } 1 \\
\text { adjective }\end{array}$ & díri ábùr & diríbure & 'the pot is big' \\
\hline $\begin{array}{l}\text { nominal pr. } \\
\text { with class } 2 \\
\text { adjective }\end{array}$ & inam ádér yéhè & inamé der & 'the boy is tall' \\
\hline
\end{tabular}

${ }^{19}$ See 1.3 .2 .

203 pers sing is used instead of the normal concord 3 pers pl. The 2 pers pl however is not homonymous with the corresponding singular in the same context. I think therefore that one cannot speak of a restrictive paradigm as Andrzejewski has for Somali or of an impoverished conjugation [Hetzron 1974:275]. 
2.4.2. Realizations of the -pfm. Here we have to distinguish between realizations which are phonologically conditioned and realizations which are morphologically conditioned.

2.4.2.1. Phonologically conditioned realizations. The relevant conditions are the following:

- the -pfm suffixed to a noun which ends in a consonant is é : (37) inamé yimi 'the boy came'

- the -pfm suffixed to a noun which ends in a high tone vowel has $\varnothing$ realization:

$\begin{array}{llll}\text { (38) an komboró arge } & \text { 'I saw the stools' } & \text { (komboró 'stools') } \\ \text { ico celé yimatin } & \text { 'they came yesterday' } & \text { (celé 'yesterday') }\end{array}$ - the -pfm suffixed to a noun which ends in a low tone vowel deletes but the high tone of $\dot{e}$ is left behind on the final low tone vowel of the preceding noun:
(39) an i I dáwá sogade
'I bought medicine'
(i ldáwà 'medicine') kolósó yamit
'perhaps he will come' (kolósò 'perhaps')

Note that there are several very similar suffixes in Rendille, which differ however in tone and/or different allomorphic behavior in vowel environment. The essence is that -̀̀ (feminine surface subject marker) disappears altogether, -è (one of the plural markers) is realized as -nye after vowel, -e (copula) remains while the preceding vowel disappears, and -é (-pfm) deletes, but its high tone is left behind on the final vowel of the noun in focus.

2.4.2.2. Morphologically conditioned realizations. The relevant conditions are the following:

- the -pfm realizes as nyé after the copula -e (-e is a subjunctive form, i.e. occurs in subordinate clauses only).
(40) inankí bure inanki burényé yimi
'the boy who is big' (bur +e, copula)
'the big boy came' (focus on bure)

- the -pfm realizes as nyé after the negative of the copula -me . mé (raised tone) was found as well. 
(41) abártána ?

mélè, abártéymé

abártéymenyé, abártáhà

kané dona, kúsmenyé 'is it your mother?'

'no, it is not my mother'

'it is not my mother, it is your mother'

'I want this one, not that one'

- the -pfm realizes as né after nouns followed by the demonstrative -a 'this, these'. 21

(42) hertà

$$
\text { hertané timi }
$$$$
\text { intà }
$$

us intané kajira

mántà

us mantané yamit 'these warriors'

'these warriors came'

'here'

'he is here'

'today'

'he will come today'

2.4.2.3. Different types of subject noun phrases examplified. Eight possible noun phrases in subject position will be considered, with the focus on the subject version on the left-side and the focus on the predicate version on the right-hand side. Note that the +pfm realizes also unmarked focus.

(43) simple noun

inamé yimi

'the boy came'

maxábálé bun karisa

'the woman is cooking coffee'

(44) pronoun

átiné bahási tigasin

'you (pl) killed the lion' inam áyimì

'the boy came'

maxabálè bun ákárísà

'the woman is going to cook coffee'22 átìn bahási átigásìn

'you killed the lion'

${ }^{2} \mathrm{l}_{\text {Another }}$ possibility of analysis is not to consider né an allomorph of $\underline{e}$, but to assume another stem to which it is attached: an instead of a . Heine [1976] has a Proto-Sam demonstrative root *-an besides * ${ }^{*} a$, both having the meaning of being near. In present day Rendille we find a nominal demonstrative kàn 'this one'. It is therefore possible to assume that the $n$ has disappeared in the adjectival demonstrative, but surfaces again in the focus form.

${ }^{22}$ See 2.5 .1 for difference in aspect. 
(45) noun + demonstrative

etusé min gube

'that man burnt the house'

étùs min ágúbè

'that man burnt the house'

(46) noun + possessor noun

úrubi biceté inta kajira úrubi bicet inta ákájirà

'the calabas̄h of water is here''the calabash of water is here'

(47) noun + pronominal possessive

abaháya gàl mala

abahèy gal ámálà

'my father is milking the camels' 'my father is going to milk the camels'22

(48) noun + adjective

eti herti deré buje

'the tall warrior died'

eti herti der ábújè

'the tall warríor died'

(49) noun + relative clause

eti herti cele yimié, buje etí herti cele yimi, ábújè

'the warrior who came 'the warrior who came yesterday died' yesterday died'

(Note that in the sentence on the left hand side the -pfm is attached to the last constituent of the relative clause, which is always the predicate.)

2.5. Interaction of Tense/Aspect, Mood and Focus.

2.5.1. Tense/Aspect. As in many other languages there is no sharp distinction between Tense and Aspect in Rendille.

(50) áyamit

$$
\left\{\begin{array}{l}
\text { 'he comes' } \\
\text { 'he is coming' } \\
\text { 'he will come' }
\end{array}\right\}
$$

(51) áyimi $\quad\left\{\begin{array}{l}\text { 'he came' } \\ \text { 'he has come' }\end{array}\right\}$

Adverbs and verbal affixes may be used to express further distinctions of Tense and Aspect. It appears that Focus structure can interfere, but this needs further investigation. 23

23 It may be that á functions not only as a focus marker, i.e. brings the action into focus, but also as an aspect marker. Secondly, if the predicate contains an object, one can ask: Is the entire verb phrase asserted, i.e. in focus, or only the complement? 
(52) us min ádisda 'he will build a house' (this means 'he will marry') us miné disda 'he is building a house' (with ro further implications) an tólà rié mala 'I am milking the goats now' an sáhtà $r i$ ámala 'I shall milk the goats tomorrow'

2.5.2. Tense/Aspect and Mood. The following data have been taken from a class $2 a$ verb, where there is no interference of phonological environment. All other verbs show the same pattern (underlined affixes are relevant to the present discussion).

Affirmative Negative

Imperative

$\mathrm{S}$ girdam 'dance!' ágirdamin

Pl girdama ágirdamina

Indicative

Pres/Fut

Past/Perf ágirdama ágirdame

( 1 pers) mágirdamo

(1 pers)

Subjunctive

Pres/Fut

girdamo

(1 pers) igirdamin

(invariable)

Past/Perf

girdame

(1 pers)

igirdamin (invariable)

The following observations can be made:

- The Imperative Singular (aff.) has the form of the verb stem. The Imperative (neg.) has á- prefixed, presumably to distinguish it from subjunctive forms which show the same suffix - in.

- The indicative forms have either á- (aff.) or má- (neg.). Má is presumably me+á. Suffixes $-a$ and $-e$ are found in the affirmative, markers respectively of Pres/Future and Past/Perfect; in the negative we find $-o$ and $-i n$, which are in absence of má- subjunctivc suffixes. (-o aff. and - in neg.)

- The subjunctive forms show absence of á- and má-. The negative marker is i-.

2.5.3. Indicative and Subjunctive. Lyons [1968:312] says, "The traditional term 'subjunctive' is revealing: it comes from the Latin translation of the Greek word for 'subordinating' and shows that for the traditional gramarian the subjunctive was the mood of subordination par excellence. The question arises now whether this subjunctive is correctly 
described as a mood. More generally: what is the connection between mood and subordination? etc."

For Rendille it seems to be the case that:

- The subjunctive suffixes occur in main sentences accompanied by á- and má only.

(54) ágirdamin

xoro águbno

ásogatani

mági irdamo

mági rdamin 'do not dance'

'let us burn firewood'

'let us buy'

'I do not dance'

'I did not dance'

inam mwalimu méheynan 'the boy was not a teacher' (ma $\rightarrow$ me/__ A)

- The subjunctive occurs in main sentences without á- or máafter a subject noun or noun phrase in focus. It will be shown however that these main clauses are in fact main clauses with an embedded reduced relative clause (see 2.6 below). In these cases the negative marker is i-, which is an indicator of subordinate clauses.

$\begin{array}{lll}\text { (55) inamé yimi } & \text { 'the boy came' } & \text { *inamé áyimi } \\ \text { inamé igirdamin 'the boy did not dance' *inamé mágirdamin }\end{array}$

- The subjunctive occurs in subordinate clauses (only relative clauses are considered here, but it may be that in Rendille subordinate clauses are relative clauses marked by a relative clause marker) without á- or má- only. The fact that á- and má- $(+p f m)$ do not occur in subordinate clauses, is in agreement with the fact that the predicate of a restricted relative clause, which always contains presupposed knowledge, cannot be new information.

(56) inanki inta kajiro, áxanan yehe 'the boy who is here is ill' 
(56) cont.

an atiki masiso dona 'I want you to finish' (verb stem masi)

*ámasiso

*ámasisa

Note that after verbs like arg 'see' and agard 'know', two constructions are possible:

(57) an árga maxabálè îreta 'I see the woman going' (two main clauses, each with + pfm)

an maxabás Í irati árga 'I see the woman going' (main clause with embedded rel. cl.)

\subsubsection{Interrogative.}

2.5.4.1. Yes/no questions. Occurrences of the -pfm are found as well as occurrences of the $+p f m$, but only one at a time.

(58) Naliyoné yimi?

'did Naliyon come?'

(59) Naliyon áyimi?

'did Naliyon come?'

2 5.4.2. Wh questions. In Wh questions the +pfm is ungrammatical. This is explained by assuming that Wh words have built-in focus (cf. Hetzron [1965:118; 1969:31-33, 74]). Note that they always have prominence.
(60) ayó yimi?
'who came?'
*ayo áyimi?
min ciróh masata?
'when will the house be finished?'
*ámasata yeie sagóh ifiride kùl mé?
'how did the boys escape?'
* ifiride
améh hagica?
'where is the container?'
*áme
inankóh iderneynan? 'which boy is not tall?'
*áhagica
(note subj. form)
After Wh words we find the same constructions as after -pfm and as in rel. clauses, i.e. we find verb forms which have characteristic sub- junctive markers.
2.5.5. Negation. We have seen that negative verb forms share with sub- junctive verb forms the same suffixes (one exception is the subjunctive Past/Perfect). Imperative (neg.) and Indicative (neg.) have, however,

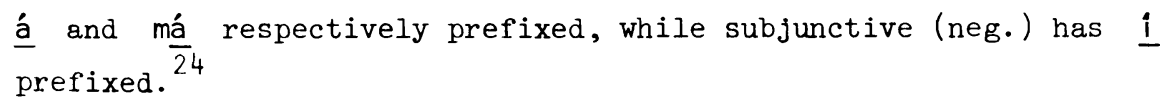

${ }^{24}$ Note that in Rendille the words for 'yes' and 'no' are respectively áa and mélè $(m e+l a)$. 
As with Focus the scope of assertion is relevant, likewise with negation the scope of negation is relevant. There are for example two ways to say 'I do not want any thing' in Rendille:

(61) an walah mádono (statement, unmarked focus)

(62) walak an dono mélè (felicitous answer to the question: 'what do you want?', note subj. form dono)

Further investigation on the matter is needed.

2.6. An attempt to derive the -pfm from the relative clause marker + copula $e$. This amounts to saying that the $-\mathrm{pfm}$ is in fact a cleft sentence construction but not regarded as such any longer by the native speakers of the language. One may assume that restructuring has taken place. Such a restructuring hypothesis is strengthened by the fact that there is one odd verb form occurring after a subject nominal in focus, which has characteristics of both indicative and subjunctive, i.e. a form of class 2 verbs.
(63) inamé girdama
'the boy is dancing'
ayó girdama?
'who is dancing?'

but

\section{(64) inanki girdamo 'the boy who is dancing'}

Girdama is a subjunctive form in so far as it has not á prefixed, but it is not a subjunctive form in so far as it has the indicative ending -a and not the subjunctive ending -0 .

2.6.1. Phonological argument. We have kíye, Aịe, and tíye in free variation with $\mathbf{k i}, \mathbf{h} \underline{i}$, and $+\underline{i}($ see 1.4.3). It is proposed here that kiye, hiye, and tíye are realizations of underlying $k \underline{i}+e, h \underline{i}+e$, and $+\underline{i}+e$ respectively, e being subjunctive present of the copula. At some time kiye, hiye, and tiye must have lost their gender marking, which is not essential to the meaning; also $\underline{i}$ was lost before the copula, but its prominence remained. The hypothetical process of the loss of $i$ is made plausible by the existence of a similar still productive process:

(65) Naliyoné mwalime 'Naliyon is the teacher' (mwalímu + e, copula) 
The foregoing hypothesis would explain the prominence of the $-\mathrm{pfm}$. Hence the following development may be assumed:

$$
\left\{\begin{array}{l}
k \text { iye } \\
\text { híye } \\
+ \text { íye }
\end{array}\right\} \quad \rightarrow \quad \text { iye } \rightarrow \quad \rightarrow \quad \text { é }
$$

2.6.2. Syntactic arguments. We have seen under (56), (55) and (60) that we find identical clause structures following relative clause markers, subject nominals in focus and Wh words, except for a form like girdama (see 2.6). Also, we mention once again that the -pfm cannot occur on a noun in isolation (see (33)). This restriction is in agreement with our hypothesis that the -pfm is derived from a relative clause marker, i.e. something must follow.

2.6.3. Semantic argument. Restrictive relative clauses contain presupposed knowledge, i.e. knowledge shared by speaker and hearer. Constructions following a nominal in focus also contain presupposed knowledge. In Rendille non-restrictive relative clauses appear to be expressed by main clauses juxtaposed to other main clauses. Coordination need not be overtly expressed (see (57)).

2.7. Beyond the clause. It was mentioned before that an information unit may include more than one clause (see 2.1.2). This is precisely what is the case where a clause apparently contains no focus marker at all. 25

(67) inámè timiyé, inam gudubte 'the girl came and beat the boy' (Although there are two clauses, there is one information unit with one -pfm attached to the last constituent of the first clause. The clause thus, if in focus, has the -pfm attached to the last constituent which is the predicate; here, timiyé. In the second clause, inam gudupte, there is no other focus marker.)

${ }^{25}$ Another particle, la, was found, which apparently draws attention to a new topic. Example: inámè adi xabté, adila kirte, babe 'the girl was looking after the goats, then the goats started to run and got lost'. (adi 'goats') Cf. also mélè 'no'. 
2.8. Why two focus markers and not simply cleft sentence constructions? I would like to adhere to the focus structure analysis as outlined above and not treat cleft sentence constructions and verbal prefix á as two unrelated phenomena for the following reasons:

- á is not a mere verbal prefix, which perhaps indicates aspect (see 2.5.1), since it is obligatory when the predicate is not accompanied by a noun or noun phrase and in that case may indicate different aspects. The predicate contains then the new information, the presupposed part of the message being omitted. In cases where the predicate is accompanied by noun phrases, it is still obligatory if there is no -pfm attached to any of the latter.

- $\underline{e}$ and á are in complimentary distribution in the information unit.

- $e_{\text {é }}$ is no longer considered a relative clause indicator by the native speakers, which is suggested by the absence of $\mathrm{ki}, \mathrm{Ai}$, and $+\mathbf{i}$, and by ambiguous forms such as girdama (see (63)). Girdama represents the class of verbs to which the great majority of Rendille verbs belong.

\section{$\underline{\text { REFERENCES }}$}

Bell, C.R.V. 1953. The Somali Language. London: Longmans, Green and Co. Fillmore, C. 1970. "The case for case." In E. Bach airủ R.T. Harms (eds.), Universals in Linguistic Theory, pp. 1-90. London: Holt, Rhinehart and Winston.

Fleming, H.C. 1964. "Baiso and Rendille, Somali outliers." In Rassegna di Studi Etiopici 20:35-96.

Givón, T. 1975. "Focus and the scope of assertion: some Bantu evidence." Studies in African Linguistics 6:185-205.

Greenberg, J.H. 1966. "Some universals of grammar with particular reference to the order of meaningful elements." In J.H. Greenberg (ed.), Universals of Language, pp. 73-113. Cambridge, Mass.: M.I.T. Press.

Halliday, M.A.K. 1967-8. "Notes on transitivity and theme in English." Journal of Linguistics $3: 38-81,199-244 ; 4: 179-215$. 
Halliday, M.A.K. 1970. "Language structure and language function." In J. Lyons (ed.), New Horizons in Linguistics, pp. 140-65. London: Penguin Books.

Heine, B. 1975/76. "Notes on the Rendille language (Kenya)." Afrika und Ubersee 59:186-223.

Heine, B. 1976. The Sam Languages, a History of Rendille, Boni and Somali. Kölner Beitrage zur Afrikanistik, Volume 7.

Hetzron, R. 1965. "The particle bàa in Northern Somali." Journal of African Languages $4: 118-30$.

Hetzron, R. 1969. The Verbal System of Southern Agaw. University of California Publications Near Eastern Studies, Volume 12. Berkeley and Los Angeles: University of California Press.

Hetzron, R. 1974. "An archaism in the Cushitic verbal conjugation." 4 Congresso Internazionale di Studi Etiopici, 2, pp. 275-81. Rome: Accademia Nazionale dei Lincei.

Lyons, J. 1968. Introduction to Theoretical Linguistics. Cambridge: Cambridge University Press.

Oomen, A. 1977a. "Aspects of Rendille grammar with special reference to focus structure." M.A. thesis, University of Nairobi.

Oomen, A. 1977b. "The adequacy of the features tongue root position, high, low and back, in a comparison of aspects of Rendille and Somali phonology." Ms., University of Nairobi.

Stewart, J.M. 1967. "Tongue root position in Akan vowel harmony." Phonetica 16:185-205.

Tucker, A.N., and M.A. Bryan. 1966. Linguistic Analyses: the Non-Bantu Languages of Northeastern Africa. London: Oxford University Press for the International African Institute. 
Personal Pronouns

-Focus
an(ì)
at(ì)
us(ì)
icè
nàn
innò
atìn
icò

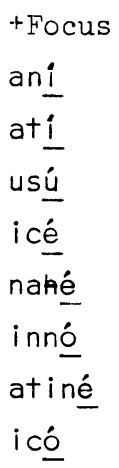

$\underline{\text { Pronominal Adjectives }}$

-Focus
-èy
-àn
-ìs
-icè
-enyò
-èn
-ìn
-icò

+Focus

-áyà

-áhà

-isà

- i cédà

-ányà

-énà

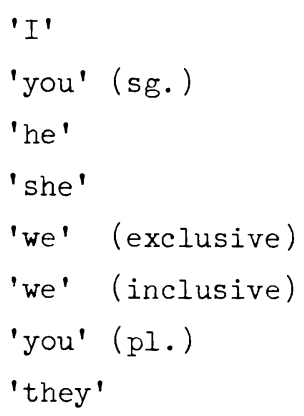

-icò

-ínnà

-icódà

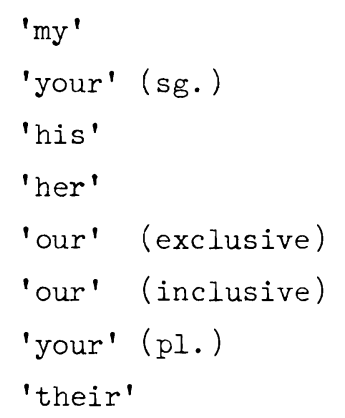

Verb Affixes for Suffix Conjugation

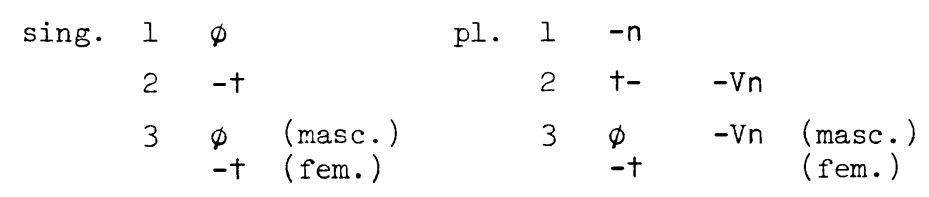

Verb Affixes for Prefix Conjugation

$$
\begin{aligned}
& \text { sing. } 1 \varnothing \\
& \text { pl. I n- } \\
& 2 \text { t- } \\
& 2+--V_{n} \\
& \begin{aligned}
y- & \text { (masc.) } \\
& t-(\text { fem. })
\end{aligned} \\
& \begin{array}{ccc}
y- & -V n \quad & \text { (masc.) } \\
& t- & (\text { fem. })
\end{array}
\end{aligned}
$$


Verbs Taking the Prefix Conjugation

$a^{\prime} i$ alternation in the stem:

\begin{tabular}{|c|c|c|c|}
\hline amit & 'I come' & $i m i$ & 'I came' \\
\hline arid & 'I run' & irid & 'I ran' \\
\hline agis & 'I kill' & igis & 'I killed' \\
\hline asil & 'I lay down' & isil & 'I lay down' \\
\hline yal & $\begin{array}{l}\text { 'it is' } \\
\text { (existence) }\end{array}$ & yil & $\begin{array}{l}\text { 'it was' (not of living } \\
\text { things) }\end{array}$ \\
\hline ehe $(a+e ;(A)$ & 'I am' & ehe $(i \rightarrow e / \ldots)$ & 'I was' \\
\hline ateh & 'I become' & iteh & 'I became' \\
\hline
\end{tabular}

a/ $u$ alternation to the stem:

$\begin{array}{llll}\text { abhub } & \text { 'I drink' } & \text { ubhub } & \text { 'I drank' } \\ \text { aham } & \text { 'I eat' } & \text { uham } & \text { 'I ate' } \\ \text { abud } & \text { 'I can' } & \text { ubud } & \text { 'I was able' } \\ \text { amut } & \text { 'I die' } & \text { umuy } & \text { 'I died' }\end{array}$

Past form only:

idah 'I said'

an idóh idah 'what have I done to ...?' (approximate meaning) 
Short illustrative text, giving examples of Focus in context.*

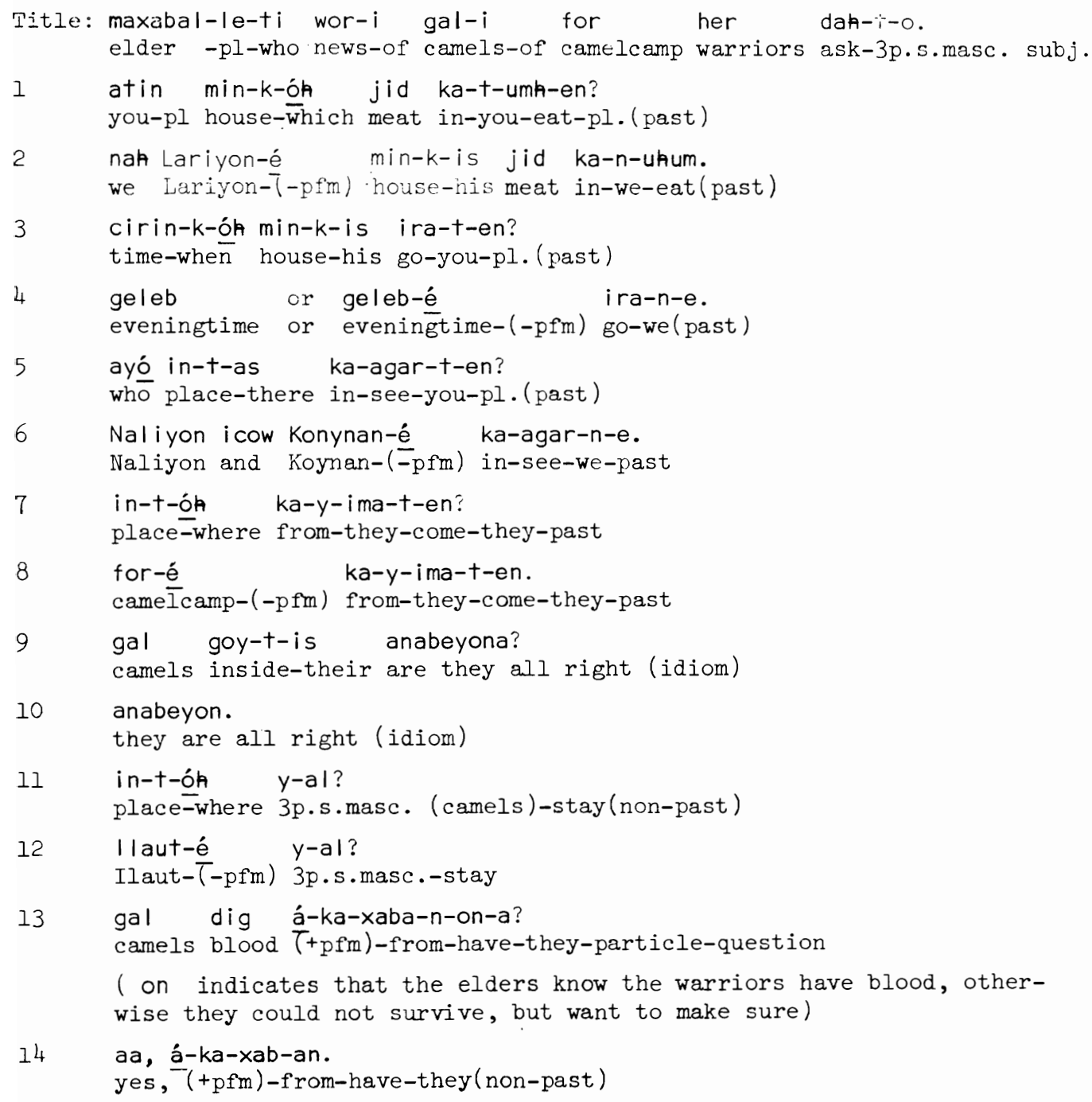

※one/stress is not narked except on the focus markers. Morphological sezmentation is marked $b_{i}$ nyrhens. 
15 guram á-xab-a?

migration $T+p f m$ )-have-3p.s.masc. (camels)

16 me-le, yax á-jir-a.

no, grazing $(+p f m)-$ be-3p.s.masc.

17 gal yax á-ka-darg-a?

camels grazing $T+p f m$ )-with-be satisfied-3p.s.masc. (camels)(non-past)

18 aa, á-ka-darg-a.

yes, $T+$ pfm)-with-be satisfied-3p.s.masc. (non-past)

19 ged-óh aron-a?

time-how long stay without water-3p.s.masc. (camels)

(The elders ask this question to test the truth of the warriors'

statement. If the grazing is indeed good, the camels can stay with-

out water for 12 days. If it is bad, for 6 days only.)

20 iben tomon icow kow-é, lama-ka wor ka-tol-a,

days ten and one-(-pfm) two-particle well place-stand-3p.s.masc. (camels)

21 in-t-óh y-abhub?

place-where 3p.s.masc.-drink(non-past)

$22 \quad$ Ngurunet-é $\quad y$-abhub.

Ngurunet-T-pfm) 3p.s.masc.-drink(non-past)

\section{Translation}

Title: Story about elders who ask two warriors about the camels in the camelcamp

At which house did you eat meat?

2 We ate meat at Laryon's house.

3 When did you go to Laryon's house?

4 We went at eveningtime.

5 Who did you see there?

6 We saw Naliyon and Koynan.

7 Where did they come from?

8 They came from the camelcamp.

9 How are the camels?

10 They are all right.

11 Where are they staying?

12 They are staying at Ilaut.

13 Do they get enough blood from the camels?

14 Yes, they get. 
15 Do the camels move?

16 No, there is grazing.

17 Are the camels satisfied with the grazing?

18 Yes, they are.

19 How long do they stay without water?

20 Eleven days, on the twelfth day they stand at the well and drink.

21 Where do they drink?

22 They drink at Ngurunet. 

PAPERS FROM THE EIGHTH CONFERENCE ON AFRICAN LINGUISTICS

University of California, Los Angeles, April 1-3, 1977

The following papers were presented in the Afroasiatic and Bantu Syntax Working Groups at the 8 th Conference on African Linguistics. 



\section{'AND WHAT ABOUT $\ldots$..' - TOPICALISATION IN HAUSA \\ Philip Jaggar \\ Universität Hamburg}

\section{Introduction}

This paper examines the transformational principles and operations involved in the topicalisation, within simplex Hausa verbal sentences, of both nominal and verhal elements. ${ }^{1}$ Several accounts of the process of focus, also considered here, have appeared for Hausa, including major studies by McConvell [1973] and Schachter [1966, 1973] and some references in Parsons [1963]. There are also a number of descriptions available of similar phenomena in related Chadic languages, including, inter alia, Newman [1970, 1974], Schuh [1971, 1972], Burquest [1973], and Hoskison [1975]. However, apart from some rather brief reports in the latter works, in addition to one paper by Jaggar [1976], no serious investigation of the equally important but very distinctive feature of topicalisation has yet been published for this group.?

The focus to date appears to have been on focus as it were, and the aim of this present study is to help remedy this situation. In the first section, the salient semantic properties of the two processes are briefly compared. However, because topicalisation, like focus, is structurally expressed in Hausa, the burden of this analysis is concerned with the syntax of topicalisation. In order to highlight the crucial characteristics of topicalisation, its syntactic behaviour is contrasted with that of focus where variations in behaviour make such a contrast illuminating and relevant

$I_{\text {This paper has its roots in undergraduate work done with F.W. Parsons }}$ at the School of Oriental and African Studies, University of London. I would like to thank F.W. Parsons, and also David Arnott, Paul Newman, and Russell Schuh for their valuable criticisms and comments on earlier versions of this article. I am also grateful to Mahmud Imam, Nuhu Sanusi, and Abdu Bichi, all of whom speak the Kano dialect of Hausa, for their help. Any errors and weaknesses are of course entirely my own responsibility.

${ }^{2}$ Parsons himself has put together some characteristically copious but unpublished notes on the subject. He refers to the phenomenon as "Thematic Emphasis", a term borrowed in fact from Arnott [1970]. Arnott [1964] was, along with Welmers [1964], one of the first scholars to call attention to the fact that different types of emphatic transform co-exist in West African languages. 
to the particular features under consideration. The purpose throughout is to illustrate the relationship between basic and derived sentences by formulating a set of rules which apply to the underlying strings and which account for the eventual surface forms. I hope, in this report, to show that the formation of the two construction types involves different T-rules and suggest a possible way of accounting for these dissimilarities.

By topicalisation I am referring to sentences of the following kind:

(1) '(as for $)^{3}$ Bill, I saw him yesterday'

(2) '(as for) the girl, she has run away'

As distinct from sentences (3) and (4) which illustrate focus constructions:

(3) clefting: 'it was Bill I saw yesterday'

(with the possible tag '... and not John')

(4) contrastive stress: 'the girl has run away'

(with the possible tag '... and not the boy')

2. Semantic Features

The feature topicalisation $[+T]$ is introduced as an optional stylistic device available in the natural language for transformationally altering both the meaning and constituent structure of an underlying unmarked sentence. In a topic construction in Hausa some item is lifted from a neutral sentence and preposed to position before the sentence subject. The element thereby overtly marked off as a topic or theme and introduced in English by such phrases as 'as for ...' or 'and ...' is here designated the topic element. The ensuing balance will be referred to as the comment.

From a universal semantic point of view the topic element is regarded as supplying old information in the discourse or situation, and the completive comment sentence as providing new and hence more significant information regarding the topic. This should be contrasted with focus constructions where it is the focus element which normally conveys the new data and which is thus of greater communicative importance. 4 The resumptive topic element is also interpreted as being normally fully specific in reference since it is prementioned in the discourse and hence presumed to ${ }_{5}$ e known to the interlocutors and is sometimes structurally marked as such. 5

3 Throughout this article, all optional elements, in both Hausa and English, are enclosed in parentheses.

${ }^{4}$ In Hausa, as in English, focussing basically indicates either the contrast of an item with another equivalent item in the discourse or the simple emphatic identification of an item.

${ }^{5}$ Common nouns selected as topics are optionally suffixed with the marker of previous or implied reference, the morpheme -n (masc.), -ì (fem.), -ǹ (plural). Proper names are considered specific by definition. 
Topic constructions are contextually conditioned and occur either as responses to information questions of the type posed in the title of this paper, and with which they share certain existential presuppositions, or as the continuation of a previous discourse or narration. Examination of Hausa texts and direct work with informants reveal that topic constructions are a common feature of both declarative and narrative contexts in the language. 6

\section{Syntactic Features}

Any item basically of a normal simplex sentence, whatever its function, i.e. nominal, verbal, or adverbial, may be marked [+T]. Moreover, exactly the same sentence constituents may assume the feature focus [+F]. In this respect at least, Hausa appears to be less constrained than some of its sister Chadic languages. In Tera for example, it is not possible according to Newman [1970:84] to mark either V or VP with [+F], and in Ngizim [Schuh 1972:229] only the subject may be focussed by special syntactic means. Angas, apparently, like Hausa allows any element to take the feature [+F] [Burquest 1972:31-32], and Hoskison [1975:227-34] reports that Gude allows either $[+\mathrm{F}]$ or $[+\mathrm{T}]$ of the same constituents as Hausa. Finally, in Kanakuru, Newman [1974:67] says that verbal nouns may contain [+F] since these are really nominal forms lexically related to verbs, from which I assume (?) that verbs may be marked $[+F]$ in the language.

The operation [+T] is applied most commonly in Hausa to nominal elements in the sentence and is performed by a combination of topic preposing and then either topic deletion or pronominalisation in the comment. If, as less frequently happens, a main verb or verb phrase is selected for [+T], then the fronted element is either repeated in full in the comment, or leaves a trace in the form of a pro-verb.

Figure (5a) exemplifies the surface word order of a typical declarative verbal sentence in Hausa, Hausa being an SVO language. The main thrust of this paper is concerned with topicalisation of the constituent items illustrated in this tree diagram.

(5) a.

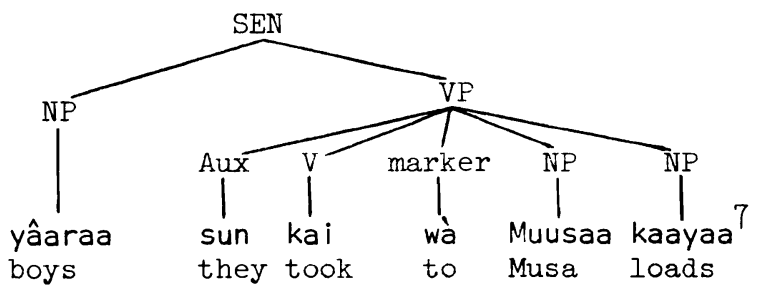

'the boys took the loads to Musa'

6 This may be compared with Tera for example, where, according to Newman [1970:89], such constructions are unnatural in ordinary speech, though employed in story telling.

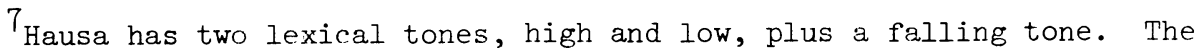


The candidates for $[+T]$ in (5a) include any of the constituent elements except the bound auxiliary subject pronoun. Examples (5b-f) illustrate the full range of possible permutations. The T-rules needed to account for these surface strings are then presented.

(5) b. NP sbj. [+T]: yâarân [+T] (dai), sun kai wà Muusaa kaayaa '(as for) the boys, they took the loads to Musa'

c. VP $[+\mathrm{T}]$ : kai wà Muusaa kaayaa [+T] (dai), yâaraa sun kai (sù) '(as for) taking the loads to Musa, the boys took (them)' or d. VP $[+\mathrm{T}]$ : kai wà Muusaa kaayaa [+T] (dai), yâaraa sun yi (shì). '(as for) taking the loads to Musa, the boys did (it)' e. NP i.o. $[+T]$ : Musaa $[+T]$ (dai), yâaraa sun kai masà kaayaa '(as for) Musa, the boys took the loads to him' f. NP d.o. $[+T]$ : kaayân $[+T]$ (dai), yâaraa sun kai wà Muusaa suu ${ }^{8}$ '(as for) the Ioads, the boys took them to Musa' Sentences $(5 b-f)$ have an underlying form which may be represented thus: (6)

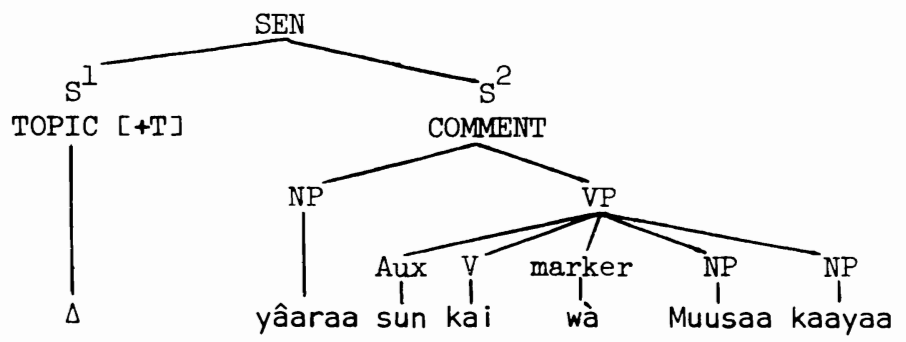

where any of the non-terminal nodes except Aux, and most typically an NP, may be dislocated leftwards to the position dominated by the feature [+T] which acts as both a syntactic and semantic marker and which is represented by the dummy symbol $\Delta$. Other appropriate transformational changes are then triggered at the same time. The process of [+T], it is suggested, results in the formation of two distinct sentences in the base component (see figure (6)), $\mathrm{S}^{1}$ being the frontshifted topic itself, and $\mathrm{S}^{2}$ the ensuing comment

transcription used here is based on Standard Hausa orthography, with the addition of a grave accent (") to indicate low tones, and a circumflex (") for falling tones. Long vowels are indicated by double letters. The first example (a) in each set is the related basic sentence. Some of the English glosses are more or less literal translations of the Hausa examples.

8 The pronoun form used here is of the disjunctive set. When a direct object pronoun follows an indirect object in Hausa, it automatically takes the form of the disjunctive pronoun. 
sentence. Syntactic, semantic, and prosodic evidence in support of this approach is presented later on in the report.

The above facts indicate that sentences which include an item marked $[+\mathrm{T}]$ in Hausa are subject to the following T-rules:

rule T.1: obligatorily transports the topic element leftwards to sentence initial position, as in $(5 b-f)$

rule T.2: optionally inserts one of the particles ai, àshee, dai, kàm, kò , kuma, kùwa, maa immediately to the right of the preposed topic element--cf. (5b-f)

rule T.3: obligatorily retains the Aux tense-aspect--cf. (5b-f)

rule T.4a: replaces an NP topic with an anaphoric pronoun in the comment sentence. This reflex pronoun agrees in number and gender with its coreferential NP and is always either 3rd person singular as in (5e) or 3rd person plural if the NP is a numerical plural as in ( $5 \mathrm{f})$

rule T.4b: deletes the NP topic in the comment sentence--cf. (5b)

rule T.5: provides that if the item chosen for [+T] is verbal, then the fronted $\mathrm{V}$ is converted into a verbo-nominal form and is then either repeated in full in the comment sentence $(5 \mathrm{c})$ or leaves a trace in the form of the finite pro-verb yi 'do, make' in the original $\mathrm{V}$ slot (5d)

The above, rather simplified T-rules, are examined in greater detail later on in the study.

Precisely the same elements are susceptible of $[+F]$ in the language, though a number of different transformational alterations would take place in the transition from deep to surface structure. Very briefly, and taking (5f) as an example sentence, the corresponding rules for focus would obligatorily frontshift the d.o. nominal selected, optionally insert the stabiliser nee in position after the preposed NP, trigger an obligatory change in the Aux aspect marker from non-relative to relative, 9 and firally delete the NP in the balance of the sentence, ending up with $(5 \mathrm{~g})$ :

(5) g. NP d.o. $[+F]$ : kaayaa $[+F]$ (nèe) yâaraa sukà kai wà Muusaa

$$
\text { 'it's loads that the boys took to Musa' }
$$

9 This takes place only where there is a distinction between non-relative and relative forms, $i . e$. the perfective and continuous aspects. In this study, many of the Hausa examples used are in the perfective aspect, though in fact the process of topicalisation is largely unrestricted as regards the verbal aspects it may occur in (see Jaggar [1976:67-68]). The stabiliser has two forms: nee (masc. and plural) and cee (fem.). It has tone polar to the preceding syllable. 
The remainder of this paper examines, in turn, the topicalisation of nominal and verbal sentence constituents of the kind exemplified above, illustrating at the same time the productivity of the T-rules thus far formulated. The mutually exclusive rules T.4a and T.4b are of particular interest and are given special considerations since they contain conditions on their obligatory and optional application depending on the construction type.

3.1. Topicalisation of nominal elements. Selection of nominals for [+T] is by far the most common process and has a high textual frequency in Hausa.

3.1.1. Subject NP [+T]. The subject function is marked by its position to the left of the main verb.

(7) a. Hali imà taa taashi

'Halima has '.eft'

b. Hal i imà $[+T]$, taa taashi

c. Hali imà $[+T]$ (kùwa), taa taashi

d. (i aa) Hali imà [tT] (kùwa), taa taashì

e. (ita) Hali imà $[+T]$, taa taashi

f. Hali imà $[+T]$ (kùwa), (ita) taa taashì

g. (ita) (kưwa) Hali imà [+T], taa taashi

h. Hali imà $[+T],(i+a)$ taa taashi

i. Haliimà $[+T],(i+a)$ (kùwa), taa taashi

[gloss for $(7 b-i)]$ '(as for) Halima, she has left'

Sentences $(7 \mathrm{~b}-\mathrm{i})$ illustrate the entire collaboration of T-rules and rull range of surface permutations which are possible when an NP assumes $[+T]$. Furthermore, as the single English gloss indicates, all the above grammatical strings are, for all practical purposes, semantically equivalent stylistic variants and convey the same information. I hesitate to say "identical", however, for two reasons, In the first place, some informants feel that the optional presence of a reflex disjunctive pronoun as in the strings $(7 \mathrm{~d}-\mathrm{i})$ lends more force to the topic nominal. And secondly, when such a pronoun occurs to the right of its coreferential NP, as in ( $7 f$ ), $(7 \mathrm{~h})$, and $(7 \mathrm{i})$, it may indicate different presuppositions by placing selective contrast on the topic, i.e. conceding the possibility that others may perform a different action.

$(7 \mathrm{~b}-i)$ reveal that when the subject NP of an unmarked sentence assumes the feature $[+\mathrm{T}]$, the basic word order remains unchanged and there is free alternation between replacement rule T.4a and deletion rule T.4b. This is assuming, as I do here, that the topicalisation rules reintroduce the NP in the base and then either pronominalise or erase the now redundant NP before It achieves surface realisation, thus generating such deep structure strings as "as for Halima, Halima has left." 
Rule T.4a optionally operates to replace the subject NP with an anaphoric pronoun of the disjunctive set, either in position to the right of the coreferential NP as in $(7 f),(7 h)$, and (7i), or to the left, appositionally as it were, as in $(7 \mathrm{~d}),(7 \mathrm{e})$, and $(7 \mathrm{~g})$. Both backward and forward pronominalisation are thus possible. Notice that the disjunctive pronoun may occur either immediately before, as in ( $7 \mathrm{~g}$ ) and (7i), or after (if) the particle optionally inserted under rule T.2. $(7 j)$ :

Moreover, if (7a) were subjected to a $[+F]$ transform, we might obtain

(7) j. (i fa) Hali imà $[+F]$ (cee) ta taashi

'it's Halima who has left'

Cf. Ngizim and Kanakuru where a subject NP marked $[+F]$ is postposed over $V$ into the predicate. In a parallel topicconstruction, however, Kanakuru uses fronting apparently [Paul Newman, personal communication].

When a subject NP contains the feature $[+T]$, and neither T.4a nor T.2 are applied, but rule T.4b is chosen as in (7b), in which Equi-NP deletion has taken place, then the only indication of [+T], in spoken Hausa at least, is acoustic pause in performance following the topic, indicated throughout with a comma, and perturbation of the normal sentence downdrift.

In the language, the structural division of an utterance into a topic and a comment seems to be a major constituent break in surface structure and results in unusual pitch perturbation. 10 This applies whatever the sentence function of the topic element. The following facts illustrate this distinctive suprasegmental feature of topicalisation. The pitch pattern of the neutral declarative sentence may be roughly represented as follows:

(8) a.

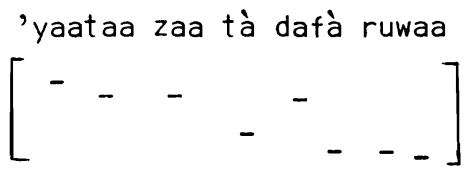

'my daughter will boil some water'

If, however, we choose to topicalise the subject NP of (8a) ('yaataa) then $(8 b)$, with the accompanying pitch contour, is one of the possible outcomes:

(8) b. 'yaataa $[+T]$, zaa tà dafà ruwaa

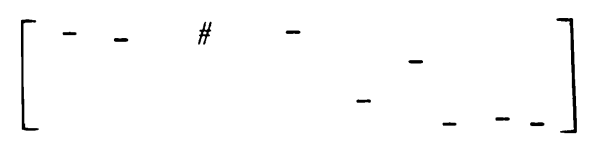

'(and) my daughter, she will boil some water'

${ }^{10}$ A feature already noted by McConvell [1973:24]. For an interesting discussion of the effect that other large surface structure breaks have on downdrift patterns in Hausa, see Meyers [1976: Chapter 3]. 
As revealed by the rough auditory representation, a pause, indicated by the symbol \#, intervenes between the topic and the comment. In ( $8 b)$ the high tone first syllable of the comment (zaa) is at the same pitch as the utterance initial high tone ('yaa) and serves to initiate a new sentence downdrift pattern. This should be compared with (8a) where the predicate initial high tone syllable (zaa) has a pitch somewhat lower than the sentence initial high tone ('yaa), since it is part of the normal neutral downdrift. In other words, the unusual features of acoustic pause and pitch perturbation may be the only criteria for disambiguating such orthographically identical surface constructions, the one neutral, the other containing a topicalised subject NP.

Furthermore, the same features may also differentiate strings which are the outcomes of different transforms, [+T] and [+F] applied to the base, but which are again. identical in surface form. Sentence (8a) for example could itself be the result of a focus transform of the same subject NP, 'yaataa, and with the same tone contour as the neutral sentence.

The suprasegmental properties associated with the topic/comment, old versus new data break, serve in effect as clues to the surface structure of the utterance. Moreover, I believe that these facts lend support to the theory that utterances in which a chosen topic is dislocated leftwards may be analysed as consisting of two full sentences, the second of which (the comment) is characterised by a new and separate sentence downdrift. I shall return to this matter shortly.

If the topic element selected is equated with a constituent NP other than the subject of the unmarked sentence, i.e. to the right of $V$, it is automatically preposed over $\mathrm{V}$ and the subject by movement rule T.I. The result in all cases is deviant surface word order.

3.1.2. Indirect object NP [+T]. Normally an i.o. is marked by the morpheme wà/ma- and appears in immediate postverbal position.

(9) a. mun dankàa wà maasinjàa wàsi iḱaa

'we entrusted a letter to the messenger'

b. maasinjàa $[+T],(a i)$ mun dankàa masà ${ }^{l l}$ wàsi ikàa

'the messenger, (well) we entrusted a letter to him'

(10) a. an yi wà kujèeraa fenti i

'the chair has been painted'

b. kujèerâr [+T] (kùwa), an yi matà fenti i

'(and) the chair, it's been painted'

${ }^{11}$ A low-level morphophonemic readjustment rule provides that the indirect object marker wà, when followed by a personal pronoun, be realised as ma-, which is the actual occurring form in the Kano dialect. 
When an indirect object NP assumes [ $+\mathrm{T}$ ], replacement rule T.4a is obligatory, at least in the Kano dialect upon which this report is based, and pronominalises the right-hand NP in the comment sentence as in (9b) and (10b). Nor does the animate/non-animate dichotomy seem to affect the choice of rule here as it does when a direct object takes [+T] (see below). This choice should be contrasted with the parallel focus construction where deletion rule $\mathrm{T} .4 \mathrm{~b}$ is obligatory, ${ }^{12}$ resulting in (10c) where no pronominal trace appears:

(10) c. kujèeraa $[+F]$ (cèe) akà yi wà fentì i

'it's the chair that has been painted'

A point worth noting here, and revealed by $(9 b)$ and $(10 b)$, is that the "exclamatory" particles $a i$ and àshee are post-pausal, i.e. the comma pause occurs after the topic but before the particle which thus initiates a new downdrift itself (see (9b)). Furthermore, these exclamatory particles mav occur to the left of the topic as may the conjunction ammaa which can, according to Neil Skinner [private communication], be used as a topic marker. "Modality" particles on the other hand, all of which may convey slightly different meanings and presuppositions, are all prepausal. Notice too that a combination of both a modality and exclamatory particle is possible, thus:

\section{(II) a. gidân yaa koonèe}

'the house has burned down'

b. gidân $[+T]$ (kùwa), (àshee) yaa koonèe

'(and) the house, (well) it's burned down'

3.1.3. Direct object NP [+T]. The d.o. is marked by post-verbal position and morphological changes in $\mathrm{V}$ termination.
(12) a. mun ga yaaròn
b. yaaròn $[+\mathrm{T}]$ (kòo), mun gan shì
'we saw the boy'
'(as for) the boy, we saw him'
(13) a. sun bùgi kàrên
b. kàrên $[+T]$ (kùwa), sun bùgee shì
'they beat the dog'
'(as for) the dog, they beat him'
(14) a. yaa wankè rìigâr
b. rìigâr $[+\mathrm{T}](k a ̀ m)$, yaa wankèe ta
'he washed the gown'
'(and) the gown, he washed it'
c. rìigâr $[+T]$ (kàm), yaa wankèe
'(and) the gown, he washed'

12 My findings on this specific point are at variance with Schachter [1973:23-24] who says that pronominalisation is in fact permissible here in the "out-of-focus clause". 
The above facts show that when the topic is under identity with a direct object NP the conditions on deletion or pronominalisation seem to be determined by whether the NP is of the category [+animate] or [-animate]. If the NP contains the feature [+animate] as in (12a) and (13a), then rule T.4a only may apply and fills the original direct object slot with a copy pronoun as in $(12 b)$ and $(13 b)$. If, however, the NP is [-animate] as in (14a), then either T.4a is again chosen giving (14b), or T.4b applies to zero the NP in the comment, yielding (14c). The majority of my Hausa informants still preferred pronominalisation to full NP reduction though, and some felt that a reflex pronoun added force to the referent NP [-animate].

However, if we apply $[+F]$ to $(14 a)$, for example, then deletion rule $\mathrm{T} .4 \mathrm{~b}$ is again compulsory, regardless of whether the NP is [+animate] or [-animate], resulting in (14d):

(14) d. rìigaa $[+F]$ (cèe) ya wankèe

'it's a gown he washed'

The admittedly Iimited data so far presented clearly indicate that there are important differences in behaviour between topicalisation and focus. Of particular interest I think is the way in which the operation of NP reduction rules differs in the two processes.

The syntactic pattern which has emerged may be summarised as follows: generally speaking, if a nominal of whatever sentence function is marked $[+T]$ and so dislocated leftwards, it is then preferably and in most cases obligatorily pronominalised in the comment sentence. If, however, the same left-hand element corresponds to the focus of the sentence, then it is deleted in the out-of-focus clause. What then, we must ask, are the conditions which determine these varying rules, and how can these dissimilarities be adequately characterised and accounted for?

The explanation, I suggest, is not to be found in the specific grammatical function of the NP as Schachter [1973:23] proposed for focus constructions at least. Rather it is to be found in the fact that the topic/ comment dichotomy constitutes a major break in structure and serves in effect to divide the utterance into two distinct sentences. In other words, the preposed topic is not part of the prime sentence in deep structure but a full sentence in its own right, as illustrated in diagram (6), and so needs pronominal support in the comment sentence. On the other hand, the focus/ out-of-focus dichotomy does not constitute such a major break since the focus itself remains part of the underlying sentence and hence does not require to be picked up with a trace. Full Equi-NP deletion thus takes place. These contrasting features may indeed be of a universal nature, and certainly seem to be reflected in English.

Furthermore, I think that additional evidence of a semantic nature may be cited in support of this proposal. In a focus construction the focus element carries a relatively heavy semantic load, and in supplying as it 
does new data of greater communicative importance, overshadows the remainder of the sentence.13 For this reason, there is no semantic motivation for picking it up with a reflex in the out-of-focus clause. In a topic construction on the other hand, the topic element bears a comparatively light semantic load, constituting old and hence less prominent information, and so needs to be picked up with a pronominal copy in the comment sentence.

It is suggested, therefore, that the choice between copying the topic NP in topic constructions and chopping the NP in focus constructions is governed by a combination of mainly structural but also suprasegmental and semantic factors of the kind outlined above.

3.2. Topicalisation of the verb or verb phrase. Attaching [+T] to $V$ or $V P$ is a legitimate if rather rare feature of the language. It is certainly not as natural or common as selecting V/VP to be the focus of an utterance or as the topicalisation of nominal items and has a very low textual frequency. The followirg sentences, however, provide pertinent and interesting examples of this feature:

(15) a. mukàn fita wani lookàci i

'we go out sometimes'

b. fitaa $[+\mathrm{T}],(\mathrm{ai})$ mukàn fita wani lookàcii

'going out, (well) we go out sometimes'

c. fitaa $[+T],(a i)$ mukàn yi wani lookàci $i$

'going out, (well) we do sometimes'

d. fitaa $[+T],(a i)$ mukàn yi i tà wani lookàcii

'going out, (well) we do it sometimes'

(16) a. sun sàyi àbinci jiyà

'they bought food yesterday'

b. sàyen àbinci[+T] (kòo), sun sàyaa jiyà

'(as for) buying food, they bought yesterday'

c. sàyen àbinci $[+T]$ (kòo), sun sàyee shì jiyà

'(as for) buying food, they bought it yesterday'

d. sàyen àbinci $[+T](k \grave{o})$, sun yi jiyà

'(as for) buying food, they did yesterday'

e. sàyen àbinci $[+T]$ (kooo), sun yii shì jiyà

'(as for) buying food, they did it yesterday'

What we see in $(15)-(16)$ is that when $[+T]$ is attached to $\mathrm{V}$ or $\mathrm{VP}$, rule

${ }^{13}$ Schachter [1973:40-45] discusses this point in some detail. 
T.I frontshifts the constituent(s) and then rule T.5 applies to convert V into a verbo-nominal form. $V$ is then either repeated in full in the comment sentence, whether intransitive as in (15b) or transitive as in (16b) and $(16 \mathrm{c})$, or it leaves a copy in the form of the finite pro-verb yi, and in the original $\mathrm{V}$ slot, as in (15c) and (16d). In the latter case, the preposed verbal noun may be repeated with an anaphoric pronoun of the direct object set following the pro-verb as in (15d) and (16e).

A corresponding $[+F]$ transform of (16a) would give (16f):

\section{(16) f. sàyen àbinci $[+F]$ (nèe) sukà yi jiyà \\ 'it's buying food they did yesterday'}

Notice that unlike [+T] transforms, $V$ may not be repeated in full when marked $[+F]$ but simply leaves the substitutive $y i$. Nor is an anaphoric element possible under $[+F]$ as it is with $[+T]$, a feature roughly comparable to the contrastive behaviour of nominals under the two processes.

4. Summary and Conclusions

The main thrust of this paper has been an investigation of the scope and importance of topicalisation in Hausa, contrasting the process at the same time with the salient features of focus. I hope to have shown that the transition from deep to surface structure involves well-established but differing operations in the two constructions types and have suggested that this surface assymetry, especially with regard to NP reduction, may be accounted for on the basis of syntactic, suprasegmental, and semantic criteria. The study shows that the complex T-rules necessary to generate topic constructions are only partially identical to those needed for focus constructions. Indeed, perhaps the only consistently applied transformational rearrangement common to both processes is that the dislocated item travels leftwards to sentence initial position.

Limited space has meant that the analysis is incomplete. No consideration has been given to topicalisation within complex, equational, or existential constructions, nor to the combination and co-occurrence of these transforms. The complicated behaviour of adverbials under topicalisation has also been ignored. Nonetheless, it is hoped that the data and analysis presented here will prove useful and stimulating to scholars interested in the syntax of other Chadic languages, in particular that of emphatic transforms. Also that this paper will prompt similar research into what is, in Hausa at least, a highly distinctive and most productive process. 
Arnott, David W. 1964. Review of The Journal of West African Languages. Journal of African Languages 3:209-12.

Arnott, David W. 1970. The Nominal and Verbal Systems of Fula. London: Oxford University Press.

Burquest, Donald A. 1972. "A grammar of Angas." Doctoral dissertation, University of California, Los Angeles.

Hoskison, James. T. 1975. "Focus and topic in Gude." In R.K. Herbert (ed.), Proceedings of the Sixth Conference on African Linguistics, pp. 227-34. Working Papers in Linguistics No. 20. Columbus: Department of Linguistics, The Ohio State University.

Jaggar, Philip J. 1976. "Thematic emphasis in Hausa." Harsunan Nijeriya $6: 63-73$.

McConvell, Patrick. 1973. "Cleft sentences in Hausa: a syntactic study of focus." Doctoral dissertation, University of London.

Meyers, Laura. 1976. "Aspects of Hausa tone." Working Papers in Phonetics, no. 32. Doctoral dissertation, University of California, Los Angeles.

Newman, Paul. 1970. A Grammar of Tera. University of California Publications in Linguistics, no. 57. Berkeley and Los Angeles: University of California Press.

Newman, Paul. 1974. The Kanakuru Language. West African Language Monographs, no. 9. Leeds: Institute of Modern English Language Studies of the University of Leeds.

Parsons, F.W. 1963. "The operation of gender in Hausa: stabiliser, dependent nominals and qualifiers." African Language Studies 4:166-207.

Schachter, Paul. 1966. "A generative account of Hausa NE/CE." Journal of African Languages 5:34-54.

Schachter, Paul. 1973. "Focus and relativization." Language 49:19-46.

Schuh, Russell G. 1971. "Reconstruction of the syntax of subject emphasis in certain Chadic languages." Studies in African Linguistics, Supplement 2, pp. 67-77.

Schuh, Russell G. 1972. "Aspects of Ngizim syntax." Doctoral dissertation, University of California, Los Angeles.

Welmers, Wm. E. 1964. "The syntax of emphasis in Kpelle." Journal of West African Languages $1: 13-27$. 



\section{WORD ORDER FUNCTION TYPOLOGY : \\ THE AMHARIC CONNECTION}

Greta D. Little

University of South Carolina, Columbia

\section{Introduction}

In her Forum Lecture at the 1976 Linguistic Institute, Sandra Thompson proposed a typology of languages based on the function of word order. 1 Her purpose was two-fold: (1) to investigate the role of word order function as a typological parameter; (2) to examine the implicational relationship between word order function and certain English structures.

Thompson cites two possible functions for word order, pragmatic and grammatical. The distinction is one which provides a continuum along which languages may range from an exclusively pragmatic function to an exclusively grammatical function for word order. These functions she explains in the following terms:

To use word order pragmatically is to use it to distinguish roughly what is "known" (the "old" information) from what is "not known" (the "new" information) in a sentence. The basic distinction between what the hearer can be presumed to know about and what he does not yet know about has been discussed in terms of a variety of parameters: old/new, known/unknown, theme/rheme, topic/comment, and definite/ indefinite. [1976:2]

She cites examples in Russian, Mandarin and Spanish where the word order signals pragmatic information. Concerning the grammatical function of word order Thompson says:

It appears that there is a small number of gramatical properties which languages may choose to signal by word order. The most obvious is the marking of subject (and to a lesser extent, object) by position in the sentence. ...There are other gram-

IThe research on which this paper is based was supported in part by a grant from the English Department of the University of South Carolina, which allowed me to participate in the 1976 Linguistic Institute. 
matical properties which word order can signal...not only basic grammatical relations, but also question, and whether a clause is main or subordinate. Beyond these properties, it remains to be discovered what other essentially grammatical information can be signaled by word order. [1976:6]

Because the positions of the major sentence elements in English are determined by their grammatical relationships virtually to the exclusion of pragmatic factors, English is chosen as the representative language for the grammatical end of the continuum, and examined for the implications of being a "word order grammatical" language. Thompson's examination reveals nine implicational features:

1. Subject Prominent

2. New Information in Sentence Initial Position

3. Use of Definite and Indefinite Articles

4. Advancement Operations = Change in Grammatical Relationships

5. Prevalence of "Structure Preserving" Operations

6. NP V NP Sequences Interpreted as SVO

7. Subject Verb Relation Preserved in Root Transformations

8. Anaphoric Pronouns Required

9. Existence of Empty Subjects

\section{Amharic as a Word Order Grammatical Language}

In this paper I look at another language at the "word order grammatical" end of the continuum, Amharic. Like English, Amharic tolerates very little variation in word order; unlike English, that order is SOV. By examining an SOV language such as Amharic, we will be in a better position to argue for word order function as a typological parameter of significance. Furthermore, we can begin to determine the degree to which these features operate independently of a particular word order. Finally, we will have a better idea of the kinds of linguistic structures we can hope to explain on the basis of such a typology as well as more precise information about remaining problems.

2.1 Subject prominent. Amharic, like English, is subject prominent. Subject prominent languages have been characterized by Li and Thompson [1976] as having subjects in initial position and as having selectional and agreement restrictions which tie the subject to the verb. Amharic has both these characteristics. In a study of Amharic neutral sentence patterns, F. P. Cotterell [1966] found the following word order patterns:

$\begin{array}{llll}\text { Subject } & \text { Object } & \text { Verb } & 16 / 16 \\ \text { Subject } & \text { Adverbial Modifier } & \text { Verb } & 38 / 50 \\ \text { Adverbial Modifiers } & \text { Subject } & \text { Verb } & 12 / 50\end{array}$

Sentences illustrating the grammatical connection between subject and verb can be found in the active/passive sentences of 2.4 below. 
2.2 New information in sentence-initial position. Like English, Amharic allows new information to serve as subjects, thus occupying the initial position.

$$
\begin{aligned}
& \text { (1) sost : wəmbəročč : ikifil : allu } \\
& \text { three : chair-pl : in-classroom : are } \\
& \text { 'Three chairs are in the classroom.' } \\
& \text { 'There are three chairs in the classroom.' } \\
& \text { (2) dabo : it'ərəp'eza : yik'k'əmmət'al } \\
& \text { bread : on table : it sits } \\
& \text { 'A loaf of bread is sitting on the table.' }
\end{aligned}
$$

2.3 Use of definite and indefinite articles. The development of articles is also related to signalling old and new information. Since that information cannot be conveyed by position in a word order grammatical language, articles to indicate definite and indefinite referents often develop to help establish the distinction. In Amharic the unmarked noun is by far the most common. However, and the indefinite article, is becoming more frequent. Use of the Amharic definite article $-u$ is more restricted than the English 'the'. Its use corresponds more to an English demonstrative; previous mention of the noun is usually a prerequisite for its use. Nevertheless Amharic articles do serve the function outlined by Thompson in helping to distinguish old from new iformation:

$$
\begin{aligned}
& \text { wambaru : } i k+f \dot{+l}: \text { allo } \\
& \text { chair-the : in-classroom : is } \\
& \text { 'The chair is in the classroom.' }
\end{aligned}
$$

(4) and : məs'ohaf : itomari: bet: allo one (a) : book : in-student: house : is 'There is a book in the school.'

2.4 Advancement operations $=$ change in grammatical relationships. Amharic advancement is somewhat different from English advancement. The passive construction is similar to that of English; the direct object is promoted to subject and thereby advanced to sentence initial (subject) position.

\section{Active/Passive}

(5) a. settyowa : Jəbəna : səbbərəčč
woman-the : coffee pot : break-3rd person fem. past

'The woman broke the coffee pot.'

b. Jabana : tasəbbəra

coffee pot : be broken-3rd person masc. past

'The coffee pot was broken.' 
Amharic also possesses a pattern of demotion, or reverse advancement:

\section{Transitive/Causative}

(6)
a. wat'u : mok'a
stew-the : be hot-3rd person masc.
'The stew is hot.'
b. and : saw : wat'u : amok'a
one (a) : person: stew-the : heat-3rd person masc.
'Someone heated the stew.'

Here the subject becomes the direct object of a derived form of mok'a, i.e. amok'a. Thus the demotion, or reverse advancement procedure moves the subject of the intransitive verb to DO position, allowing a new subject to appear, in this case the agent. In both sentences the verb agrees with its grammatical subject.

2.5 Prevalence of structure preserving operations. Thompson has suggested that the grammatical function of English word order is a reason for the abundance of structures closely resembling the svo output of Phrase Structure rules. Such structure preserving operations produce sentences like $(7 a, b$, and $\mathrm{c}$ ):

(7) a. Linda is fun to speak French with.

b. George is likely to need a ride.

c. We believe that rumor to be false.

However, Amharic counterparts to such structures are virtually nonexistent.

In Amharic the overriding concern appears to be maintaining verb final position and not having the subject too far removed from the verb. Therefore, a sentence like ( $8 a)$, where a sentential modifier has been embedded under the subject NP, has an alternative form, (8b):
(8) a. styum : wadəzih : yimat'awin : sawiyye
Siyum : to here : who is coming-def-acc : man
mas'əhaf : ayətwal
book $:$ he saw
b. wədəzih : yimat'awin : sawtyye: məs'əhaf
to here : who is coming-def-acc : man : book
styum : ayotwal
Siyum : he saw
'Siyum saw the book belonging to the man who is coming here.'

One reason this change is possible is the accusative marker $-n$ which is affixed to definite objects. Relative clauses in Amharic are definite and therefore the object is often morphologically indicated.

The most common operation in Amharic is sentence nominalization, and raising or lowering is not normally associated with the process. 
2.6 NP V NP sequences interpreted as SVO. The wording of this particular feature is clearly inappropriate for an SOV language. "NP NP V Sequences Interpreted as SOV" would be the Amharic parallel. The claim is that the grammatical role of word order is so strong that noun verb sequences which resemble the predominant sentence order will be interpreted according to that pattern unless there is an overt grammatical marker to register the difference.

In the Cotterell study, $24 \%$ of the sentences involving subjects and adverbial modifiers had a modifier + subject + verb, order. The NP's of these modifiers are in oblique cases and are marked as such, preventing any confusion about which NP is the subject:

(9)

$$
\begin{aligned}
& \text { iktfil : wambar: allo } \\
& \text { in-classroom : chair: is } \\
& \text { 'The chair is in the classroom.' }
\end{aligned}
$$

The $\dot{t-}$ prefix or preposition eliminates the possibility that $k \dot{f} \dot{+l}$ is the subject.

In Amharic a more interesting question of NP function identification is found in sentences which are embedded in object position ( $8 \mathrm{a} \& \mathrm{~b}$ ). With a stated nominal subject, the sentence would be NP NP NP V V. See (10a) below. Such a pattern can obscure the relationships, especially in more complex sentences with several modifiers. It is perhaps this possibility which motivates the OSV pattern of sentences like $[N P \text { NP V }]_{\text {obj }}{ }^{N P}$ subj $V$ in $(10 b):$

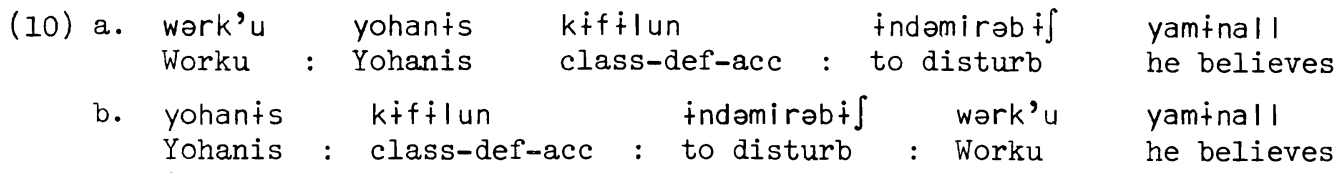
'Worku believes Johannes to disturb the class.'

2.7 Subject verb relations preserved in root transformations. Sentences derived by transformations which are not structure preserving, i.e. root transformations, involve movement away from Svo order. In English, however, an unmarked NP is not allowed to precede the verb immediately unless that NP is the subject of the verb or is clearly marked as oblique. Thompson's examples reveal the pattern, especially in (1lc), where a pronoun copy rule is necessary to maintain the subject verb pattern:

(11) a. Scrambled eggs I can't stand to look at in the morning.

b. Into the room bounced Charles.

c. He nearly ran over me, that crazy bum.

Root transformations in Amharic are limited. Aside from the OSV alternative discussed in 2.6, the other pattern which does not conform to the basic SOV structure is that involving the copula. Not only are the NP's interchangeable, but the copula itself is movable--a drastic departure from the basic Amharic verb-final pattern. Topicalization, (1la) above, 
and right dislocation, (11c) above, are both expressed in Amharic through sentences with copula verbs and the accompanying word order flexibiliby.

(12) a. yəmət'aw : siyum : now

who came : Siyum : is

'The one who came is Siyum.'

b. styum : yamat'aw : naw

Siyum : who came : is

'Siyum is the one who came.'

c. styum : now : yamət'aw

Siyum : is : who came

'Siyum it is who came.'

d. yəmət'aw : now : styum

who came : is : Siyum

'He is the one who came, Siyum.'

Therefore, in spite of its nearly rigid word order, Amharic does employ word order for pragmatic functions in the sentences above.

2.8 Anaphoric pronouns required. Thompson ties the use of pronouns for anaphoric reference in English to the fact that gramatical relations of subject and object are signaled by position relative to the verb, observing $(13 \mathrm{c}$ ) below is ungrammatical:

(13) a. Where will Joe go?

b. He will go to school.

c. *will go to school.

Amharic, however, does not use pronouns for this purpose. Instead, agreement morphemes are employed, and the Amharic counterpart to (13c) is the most natural response:

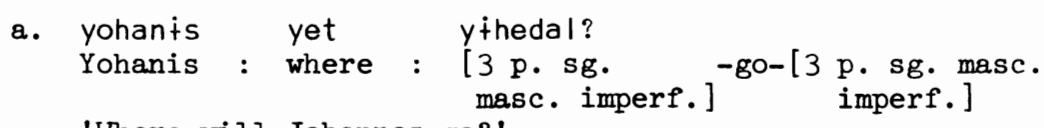

$$
\begin{aligned}
& \text { 'Where will Johannes go?' }
\end{aligned}
$$

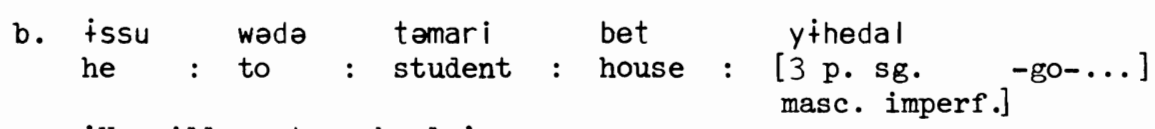

$$
\begin{aligned}
& \text { 'He will go to school.' }
\end{aligned}
$$

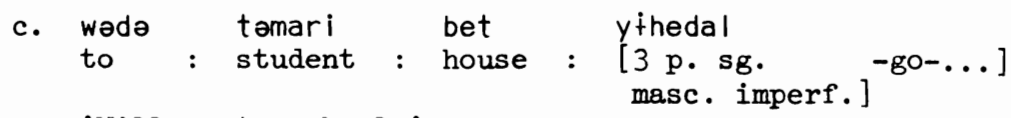

An important factor, apparently unrelated to word order function, is the extent to which grammatical agreement is indicated. Since Amharic has complete subject verb agreement, it is possible to determine the person, 
number, and gender of the subject from the verb form. Thus Amharic subjects are much more clearly indicated than English subjects.

An additional factor of importance is the fact that issu, the third person pronoun is not really anaphoric. Third person pronouns are descended from ras 'head', with the possessive pronoun suffixed. The original function was to indicate emphasis; indeed emphasis continues to be the difference between (14b) and (14c).

2.9 Existence of empty subjects. In English it is necessary to fill the subject position even though the filler has no lexical meaning:

(15) a. It's cloudy today.

b. It bothers me that so many people don't understand Southern English.

c. There is a funny smell down by the lake.

Similar constructions in Amharic occur with a limited number of verbs which are used impersonally:

$$
\begin{aligned}
& \text { rasen : yammənnall } \\
& \text { my head-acc : hurts me } \\
& \text { 'I have a headache.' }
\end{aligned}
$$

(17) kabbadə : irabotal

Kebbede, : hungered him

'Kebbede was hungry.'

The verbs have a tense aspect marker which is third person singular, but there is no NP to which it refers. English has sentences with empty subjects; Amharic has subjectless sentences.

\section{Conclusion}

How well does Amharic conform to the implicational observations that Thompson set up based on English?

The ridigity of Amharic word order centers on the position of the verb, while in English the position of the subject appears to have a greater effect on structure. As a result, Amharic allows greater variation in the position of the NP's. However, the position of those NP's seems to be motivated more by a desire to avoid ambiguity than to convey pragmatic information. The question of how this affects Amharic's position on a word order function continuum must await the investigation of other languages.

A second inconsistent area involves the designation of subject in the absence of an NP. Since Amharic verbs are morphologically differentiated according to person and number, there is no question of an Amharic sentence appearing without some indication of subject. In the sense that those agreement markers are equivalent to English pronouns, Thompson's generalizations are valid. However that equivalence is not absolute. What in English can compare to the expanded Amharic sentence with the pronoun, (14b)? 
The problem of agreement marker/pronoun equivalence will undoubtedly come up again if this typological study is extended to other languages.

In spite of the unresolved problems, Thompson's word order typology is a useful one, particularly in terms of its ability to offer explanations for the remarkable frequency of certain structures or operations in languages. For example, it provides a framework which can help explain the high frequency of the cleft sentence pattern in Amharic. Amharic scholars have noticed the phenomenon, but have not had any explanation to offer. H. J. Polotsky [1960:121] remarked:

If one ever makes comparative statistics of the frequency with which the cleft sentence is used in diverse languages, Amharic would have a good chance of taking lst place.

Thompson's typology provides a logical explanation since this pattern alone seems to bear the major role of indicating pragmatic information in Amharic.

\section{REFERENCES}

Cotterell, F. P. 1966. "The Amharic primary neutral sentence." Journal of Ethiopian Studies 4:11-33.

Li, Charles N. and Sandra A. Thompson. 1976. "Subject and topic: a new typology of language." In Charles N. Li (ed.), Subject and Topic, pp. 457-489. New York: Academic Press

Polotsky, H. J. 1960. "Syntaxe amharique et syntaxe turque." Atti del Convegno Internazionale de Studi Etiopici, pp. 117-121. Rome: Accademia Nazionale dei Lincei.

Thompson, Sandra A. 1976. "Modern English from a typological point of view: some implications of the function of word order." Forum lecture, 1976 Linguistic Institute. 


\title{
A RECONSIDERATION OF LINGALA TEMPORAL INFLECTIONS
}

\author{
Salikoko S. Mufwene \\ University of Chicago
}

\section{Introduction}

In the present paper I will propose an alternative analysis of time reference in Lingala. I will approach the subject from the semantic to the surface representation. I hope thus to discover some of the deep principles governing the use of any proper means in Lingala (no matter whether morphological, syntactic or lexical) to locate a situation in time. The approach should allow us to explain how, in time reference, the verbal forms cooperate with the adverbials to the same effect, and to account, alternatively, for the ungrammaticality of some utterances because of the violation of some of the hypothesized combinatorial principles.

My judgements will be based mostly on the creolized Lingala spoken in Kinshasa as well as in many other towns of Zaire. It may be argued, against my position here, that the difference I will point out between the previous analyses and mine are mostly a matter of dialectal divergence. I shall hurriedly note that we should actually feel less concerned here with dialectal differences than with the explanatory adequacy of the analyses in explicitly uncovering the principles underlying time reference. For I feel it is in this respect that almost all of the previous analyses have proven inadequate; such oppositions as aspect vs. tense have been introduced in the analyses without being clearly define $\overline{d .}$ In some of the analyses the identity of the so-called 'habitual' (my 'generic') is not clear as to whether it should be taken as tense, or as aspect. Many names such as 'passé antérieur, passé historique, passé récent' have been introduced without any clear specification of what they stand for, thus contributing more to the confusion, than to the clarification, of the matter. Their respective concepts have infelicitously been associated with expressions of time reference, the understanding of which conflicts with what the names say. 
In general, instead of addressing themselves to questions such as what the various expressions of time reference mean, or what particular temporal inflections ${ }^{1}$ any given linguistic device--morphological, syntactic, or lexical--happen to designate in what particular context, students of time reference have limited themselves to finding, in contextless simplex sentences, what particular morphological inflection can be associated with what time reference, and have sometimes assigned inadequate semantic values to them. They have often forgotten that a language can use both morphological inflections and other syntactic combinations altogether, to express time reference productively. And, as may be attested by the descriptions by Guthrie, [1966] Bwantsa [1970] and Redden et al. [1963], analyses of Lingala time reference not only default in depth, but are inexhaustive. Ingressive and terminative temporal inflections, for instance, are not, or are only partially, discussed.

\section{Data}

Before the above complaints steer us away from our subject, let me present the analysis of Bwantsa, since it, in my opinion, is the most explicit of them all, the most expansive and inclusive, and the one in which we may observe the evidence of most of the weaknesses I have alluded to so far.

Bwantsa distinguishes thirteen forms of time reference, all of which his analysis forces the reader to take as cases of morphological inflections. I will reproduce his own table below [1970:43], which will be interspersed with some of his own examples (marked by "B"), to which I will adjoin a few of my own (marked " $M "$ ). Translations are my own (some of which correct his misinterpretations). In the table: " $R$ " stands for root/radical, "V" for vowel, [ $\left.{ }^{\vee}\right]$ and ['] for tone, after Bwantsa's usage. The morphological division in the "Examples" column is mine, while TI in the glosses represents 'time inflection', and VP 'verbal prefix'.

${ }^{1}$ I strongly urge my readers not to confuse the concept of temporal inflection introduced here with the one of morphological inflection also used in the text. While the latter refers to the conventional surface morpheme, the forme is a semantic concept. It has been inspired by Chafe's Meaning and the Structure of Language, and I use it to designate any of the particular semantic features whose logical function is to locate the described situation in time. Temporal inflections are hypothesized as temporal features and are called 'inflections' (by analogy to surface inflections) because they operate in ways which, as Chafe puts it, "do not limit the choice of a lexical unit" [p.167-8], but simply specify the situation as to the position it occupies on the time vector and as to its internal temporal structure, e.g. in progress, termination, initiation, punctual, etc. Although always present in the logical structure, they can, but do not necessarily have to, surface in the various ways known as affixation, particular syntactic combinations, adverbs, or combinations of some or all of these. 
TABLE 1

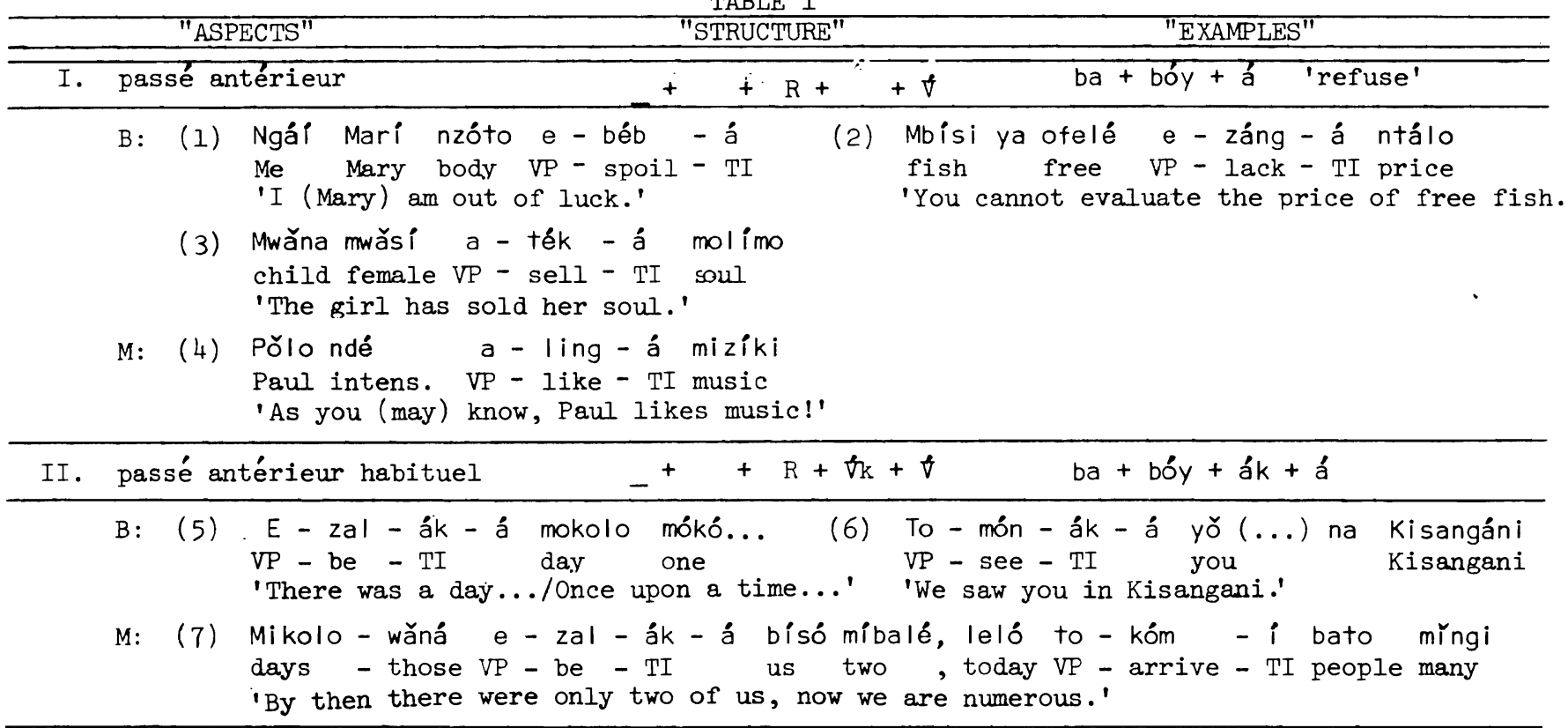

\begin{tabular}{|c|c|c|c|c|}
\hline III. & passé $h$ & storique & $+R+\forall k+i$ & $b a+b o ́ y+a ́ k+1$ \\
\hline & B: (8) & $\begin{array}{l}\text { Ba - bót - ák - i yǒ - } \\
\text { VP - deliver - TI you - } \\
\text { 'You were born on a Sunday.' }\end{array}$ & $\begin{array}{l}\text { - mokolo mwa - yenga } \\
\text { - day }\end{array}$ & \\
\hline & M: (9) & $\begin{array}{l}\text { To - món - ák - i yǒ (...) } \\
\text { 'We saw you in Kisangani.' }\end{array}$ & na Kisangáni. & \\
\hline
\end{tabular}


TABLE 1, cont.

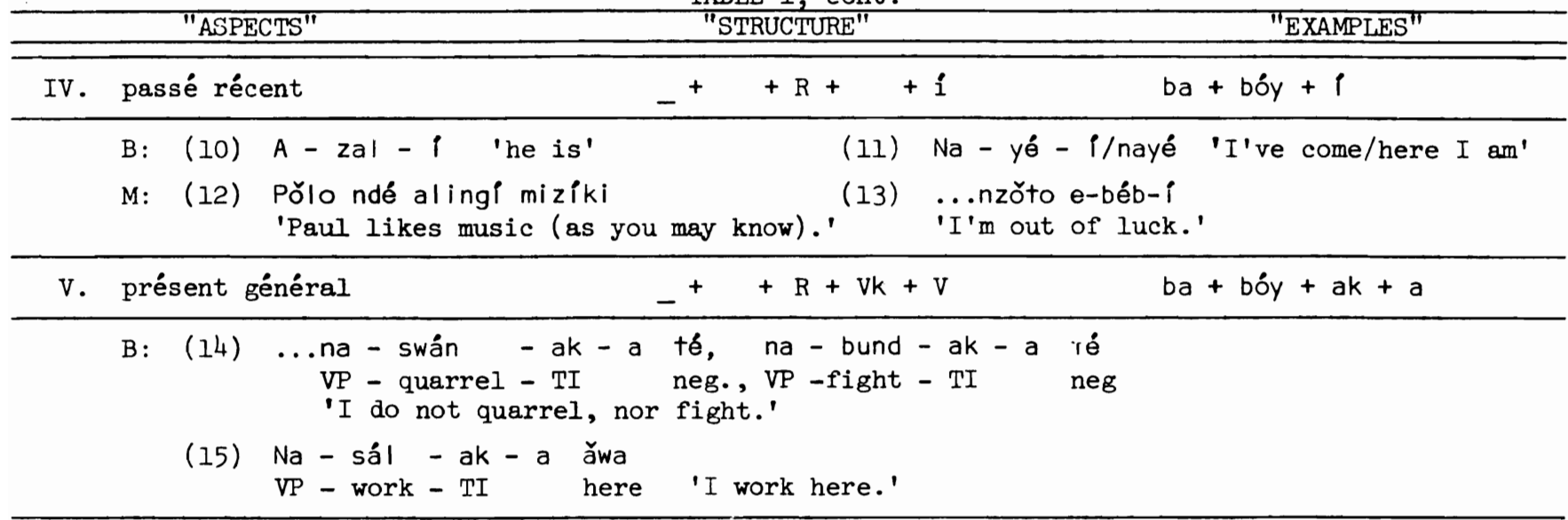

VI. présent général habituel _ + ${ }^{2} 0+R+V k+V \quad b a+k o+b o ́ y+a k+a$

B: (16) Mokili e - ko - zal - ak -a bôngó

world VP - TI - be - TI like that 'The world is/will (always) be like that.'

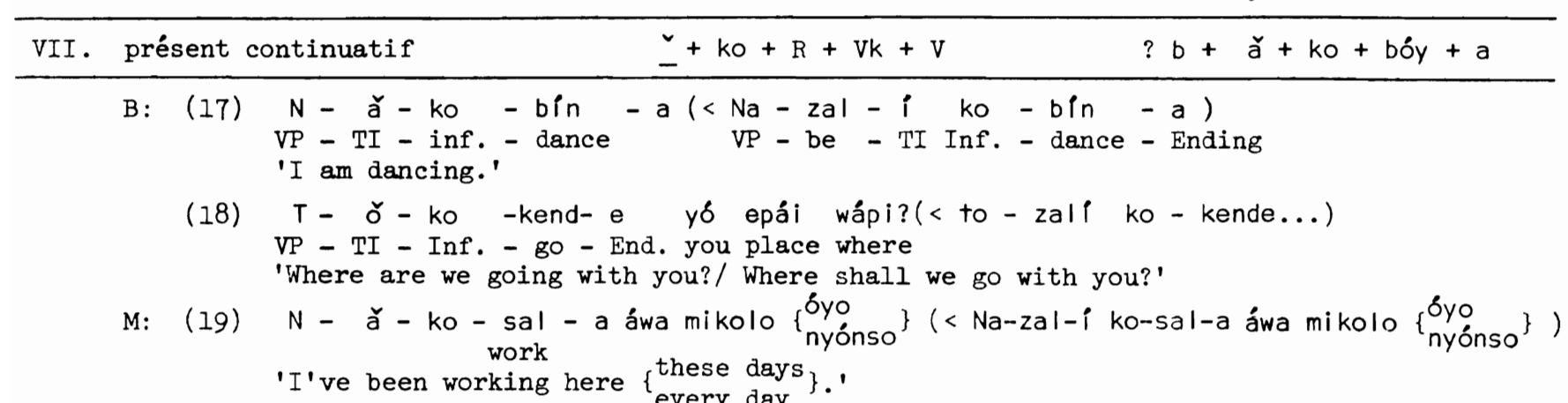


TABLE 1, cont.

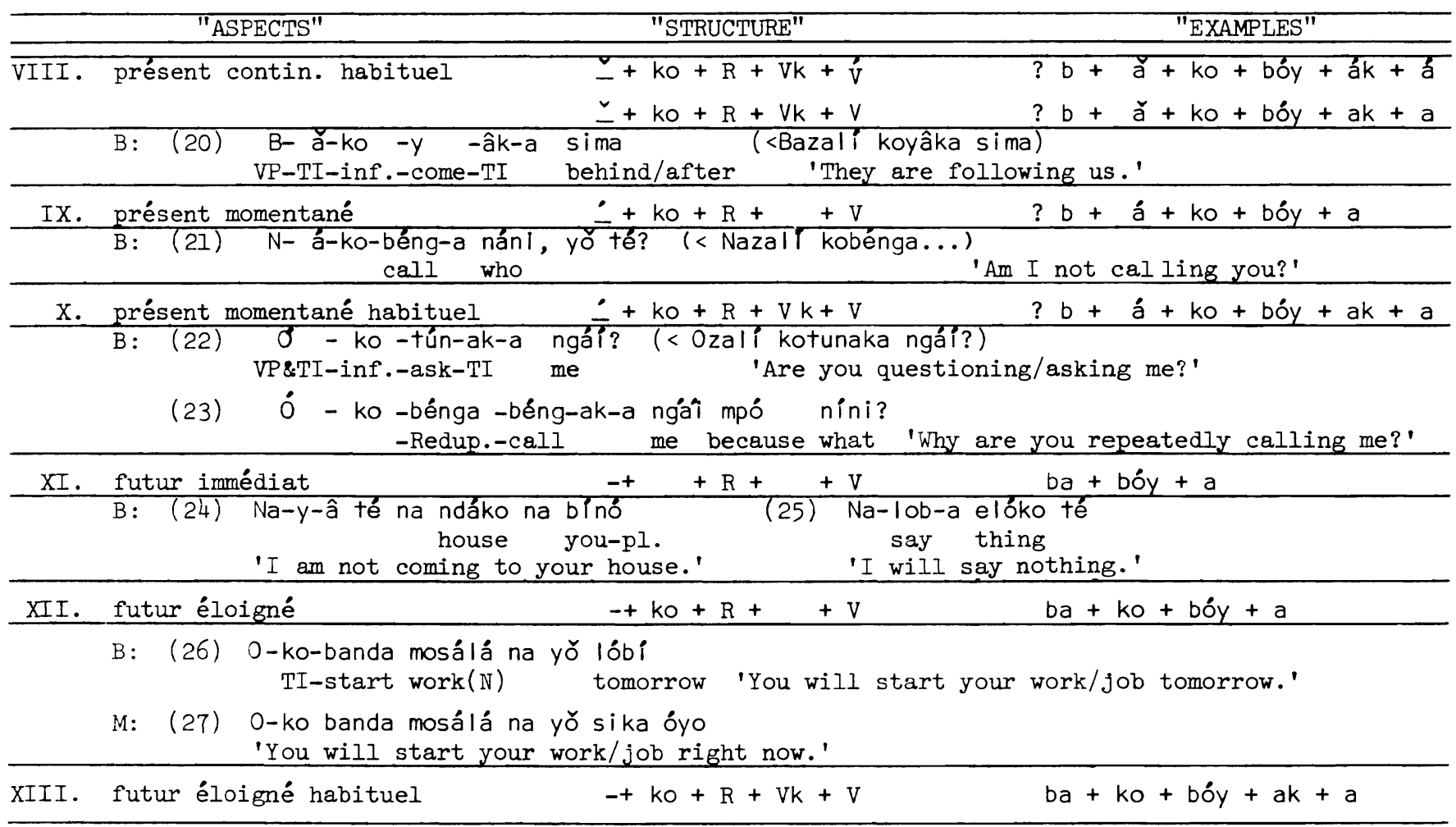

B: (28) Na-ko-tíndel-ak-a yŏ nsango na ngái
VP-TI-send -TI news me 'I will be writing to you/sending you my news.'
(29) Na-ko-bátel -ak-a mwăna na bisó
'I will be looking after our child.'




\section{Criticism and Suggestions}

Bwantsa's analysis differs from Guthrie's mostly in the names and number of particular expressions for time reference. For instance, Guthrie's "passé indéfini" and "passé défini" correspond to Bwantsa's "passé récent" and "passé historique" respectively. Guthrie also mentions a set of two "compound tenses" [1966:30] which I fail to see in Bwantsa's analysis:

(30) Na-jal-ák-á kosála ( = Na-zal-ák-á kosála ) I was working

(31) Na-sil-i kokoma finish writing 'I've finished writing.'

(30) is in fact the past counterpart of Bwantsa's (VII), which the latter may have misplaced as a case of morphological inflection, because of its present tense contraction. Neither of them has, though, considered the productive time references with -tik- 'abandon, stop', and -kóm'arrive' as auxiliaries which designate, respectively, volitional terminative and ingressive temporal inflections, as in:

$$
\begin{aligned}
& \text { Na-tik-i ko-koma } \\
& \text { 'I've stopped writing/, } \\
& \text { I'm no longer writing.' }
\end{aligned}
$$

(33) Na-kóm-i ko-koma

$$
\text { 'I've (just) started to write.' }
$$

(34)a. A-kóm-i ko-lela-lela

b. A-kóm-á ko-ylb-ak-a

'He's got into the habit of crying. 'He's got into the habit of stealing/He's become a thief.'

$$
\text { Mwana a-tik-á kol la mabelé }
$$

'The child $\left\{\begin{array}{l}\text { no longer eats } \\ \text { has given up the habit of eating }\end{array}\right.$ dirt.'

Both Bwantsa and Guthrie subordinate aspect to tense; their primary division of time reference is in terms of past, present and future. Unfortunately, this position has regrettable consequences. In Bwantsa's analysis the "continuatif"='durative', for instance, seems to be typical of present alone. And, actually, both Bwantsa and Guthrie fail to present the future counterpart of the durative in (30)/(VII)--which, by the way, Bwantsa does not signal as contracted from -zal- + infinitive. See ( 36$)$ :

$$
\begin{aligned}
& \text { To-ko-zala ko -bín-a ntángo yǒ o-ko-kóm-a/o-kóm-í } \\
& \text { VP-TI-be inf.-dance time you VP-TI-arrive } \\
& \text { 'We'll be dancing when you arrive.? }
\end{aligned}
$$

Again, because of the subordination of aspect to tense, as well as of too much concern with morphological isomorphism, Bwantsa may also be criticized for failing to realize that while his "passé antérieur habituel" without -zal- expresses "habit" in a very dubious way (see examples (5)(6)), it is, instead, the use of the past counterpart for the durative, in conjunction with the time adverbial specifying a long period of time or repetition, which is the common practice for designation of past habits 
in Lingala. This is illustrated in (37-38):

(37) To-zal-ák-i kobina $\left\{\begin{array}{ll}\text { mpókwa nyónso } & \text { na congé } \\ \text { night every/all } & \text { vacation } \\ \text { mbala na mbala } & \text { na congé } \\ \text { frequently/repeatedly } & \text { vacation }\end{array}\right\}$

'We used to dance $\left\{\begin{array}{l}\text { every night } \\ \text { frequently }\end{array}\right.$ during the vacation.'

(38) To-zal-ák-i kobima mpókwa nyónso sima papa azóngí

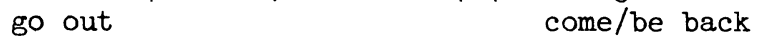

'We used to go out every night after daddy was back.'

The morphological inflections in (5-6) could have been called anything but "habituel". We will come back to this below.

I would also like to confess my failure to grasp some of the distinctions Bwantsa makes:

(a) Although he has insisted on a tone difference between (VII) and (IX), there does not seem to me to be any more than a lexico-semantic difference between examples (17-18) on the one hand and (21) on the other. Both cases seem to be instances of the present durative. Were the situation described in (21) ontologically capable of lasting as long as the situation in (17-18), I really doubt that there would be any motivation for distinguishing (2l) as "momentané"='punctual', as opposed to "continuatif". The question arises whether in such analyses we are to confuse the intrinsic aspectual property of the lexical item with the grammatical aspect as a temporal inflection.

(b) Similarly, (VI) and (XIII) fail to strike me as being different temporal inflections. It seems to me that although (VI) expresses habits, which happen to be universal, its presentation is still prospective, that is, future-oriented. As such, (VI) refers to the same time and in the same way as (XIII). The difference pointed out by Bwantsa may be epistemic rather than time-referential.

(c) I still have doubts about the traditional characterization of universal habitual/generic as a property of the present. What then should account for the difference between (15) and (19), both of which express habits? Is it just the presence of the time adverbial in (19)? Do (19) and $\left(15^{\prime}\right)$ below have more than a mere extensional coincidence:

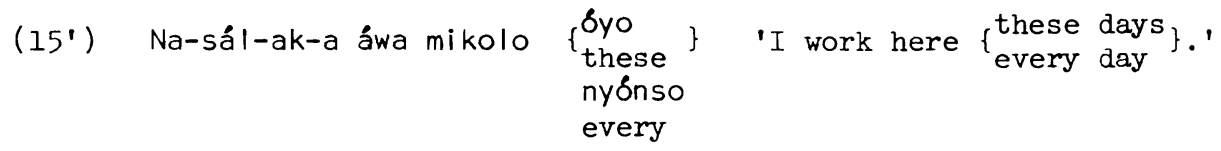

How could we account for the change of grammatical meaning (in terms of temporal inflections) for the durative in (39) and the ungrammaticality of $(40)$ ? 
(39) Na-7al-f kosála áwa lel'o 'I am working here today.' (non-habit.)

(40) *Na-sál-ak-a áwa leló ?I work (Habit.) here today.

When is a present general enough to be a habit? And when does a (present) durative qualify to express a habit?

I will conjecture that while there do seem to be habits confined to past, present and future (as illustrated in (37-38), (19), (VI), and $(X I I I)$ ), we may consider (V) as a universal generic, which transcends the division of time into the above three major categories. This alternative position will allow us to account for the actual difference in the understandings of (19) and (15') which we may try to explain in terms of scope difference. We may assume a metalanguage where " $x$ " represents the described situation, " $t$ " the time and CONTINUOUS can be interpreted as 'lasting in time', and DURING as the predicate for the time period which lasts. We could thus represent the situations in (19) and (15') as:

(19b) ( $\exists t:$ DURING $t$ ) AT( $x, t)$

'there is a period, $t$, during which $x$ (as a habit) occurs, where the habit is necessarily confined to the period'

$\left(15^{\prime} b\right) \quad(\exists x:$ CONTINUOUS $x)$ AT $(x, t)$

'there is a generic/continuous situation $x$, such that it occurs

at time $t$, where the occurrence of $x$ in $t$ is contingent.'

This enables us to account for two kinds of generics in Lingala; the one is bound to a specific time portion, and the other, more universal one, is open-ended and transcends the specified period of time.

We cannot yet forget the question of the interaction between the time adverbial in (19) and the durative, to express habit; while in (15') the time adverbial also specifies a time portion of that habit, but does not contribute to the specification of the described situation as a habit. It is just as though in (19) the time adverbial extends the time long enough to make the CONTINUOUS situation mean a habit, whereas in (39-40) the time adverbial limits the time span seriously enough to make the CONTINUOUS void of any habitual interpretation, and thus introduces the apparent clash of features in (40).

Does this suggest that generic is but an expansion of durative in time, to the point where it transcends past or present and becomes almost predictable for the future? Or does this show (as I hypothesize) that durative and generic belong to the same category, of which the durative is unmarked and the generic marked?

Whatever the case here, I would also like to draw attention to the fact that the ungrammaticality of (41) below does call for a reconsideration of the interaction of both verbal inflectional or syntactic combinatorial time reference on the one hand, and time adverbials on the other, to locate a situation in time. There is at least a need to understand 
why the combination of some adverbials with some other morphological or syntactic-combinatorial inflection leads to ungramatical utterances.

(41) *Ba-kang-ák-á ngậ lóbi l 'I was arrested yesterday.'

yet (42) is quite correct:

(42) Ba-kang-ák-i ngái lóbi 'I was arrested yesterday.'

This may become clearer after we reconsider Bwantsa's discussion of (I) vs. (IV) and (II) vs. (III). A look at examples (1-13) seems to show that not only the names may have been misgiven to these expressions, but also that: $(1,4,6)$ receive translations similar to $(13,12,9)$ respectively. These sentences differ, however, in implicatures. Indeed the latter group, which is less marked, conversationally implies a recent situation/ event. There is evidence that whenever a time reference expression (II) can be used, (III) car also be used, but not vice versa. I will show this with more examples, where time adverbials are used concomitantly with verbal forms as either supplementary or complementary temporal inflections :

(43) a. To-món-ák-i yǒ mbóla e-lek-i na Kisangáni see you year pass

'We saw you last year in Kisangani.'

b. Tomónáká yǒ mbóla elekí na Kisangani

'We saw you last year in Kisangani.'

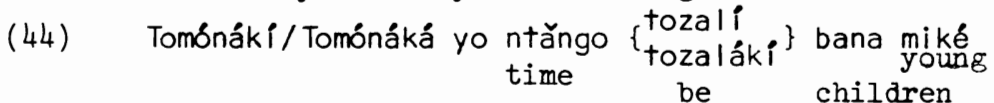

'We saw you when we were young children.'

(45) Tomónáki/*tomónáká yo $\left\{\begin{array}{l}\text { leló na ntóngó } \\ \text { sika óyo }\end{array}\right.$ 'We saw you $\begin{array}{l}\text { this morning } \\ \text { just now }\end{array}$

Although the forms with -ák-á and -ák-i translate quite identically into English, they correspond to quite different subjective attitudes towards the points of time referred to in relation to the speech event time or another landmark in the discourse. The form with -ák-á refers to a point considered as remote in the past, hence the ungrammaticality of $(45)$.

In the case of (9), by opposition to (6), we may assume that since (6) refers to a point of time considered only as remote, were the speaker more cooperative he would have said (6)--since it is unambiguous--if he had meant to refer to the remote past. Since he used (9), it must have been by necessity, to refer to recent past. Thus, the meaning of the form with $-a k_{-1} i$ is specializing, because of its coexistence with the delimiting -ák-á. But because it still has not ceased to have the remote meaning, we say it designates recent past by implicature.

Bwantsa calls (II) 'anterior', almost the equivalent of past perfect. Undeniably, in some cases it works that way. But I will show, 
after discussion of the opposition between (I) and (IV), that it does so mostly by virtue of its designating perfective anteriority, and that, as he must have noticed, the expression of anteriority is not limited to (II), since (I) has the same effect. But first we must understand how (I) differs from (IV).

Bwantsa and Guthrie call the verbal forms (I) and (IV) "past". My translations of examples $(1-4)$ and (10-14) alternate, as in Bwantsa's own analysis, between the English simple present and present perfect tenses. I can even provide more cases which seem to argue against assuming (I) and (IV) as referring to past time. Observe (46):

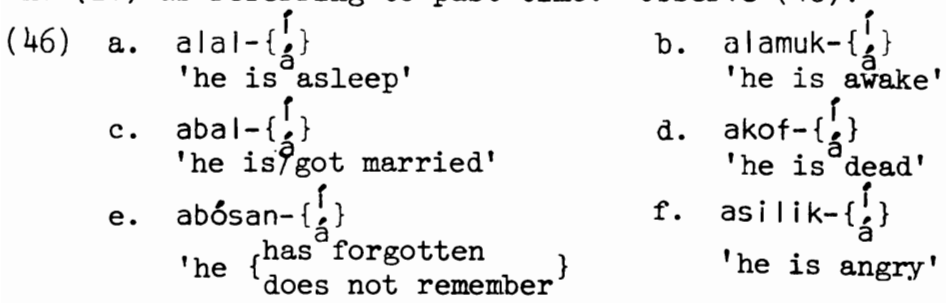

That the English present tense fits best in translating these expressions would seem to suggest that these verbal forms are time-inflected in the present rather than in the past. For cases like (3) and (11), it seems plausible to interpret the lexical meaning of the verb as specifying the beginning of the present state. If this is correct, it seems to me that we may just as well call these two forms PERFECT, in the sense defined by Comrie [1976], after McCawley [1971] and Huddleston [1969]: that this temporal inflection specifies the present relevance of a past event/situation. We could alternatively interpret them in the way suggested by Guthrie, that is, as a form of grammatical stative. (However, it seems to me that Guthrie's interpretation and the PRESENT RELEVANCE one converge rather than contradict one another.)

As with verbal forms in $-a ́ k-i$ vs -ák-á, I will assume that the verbal forms with $-R+i \#$ and $-R+a \#$ of the hypothesized perfect also differ by conversational implicature, at least the former from the latter. Let us consider $(47-48)$ :

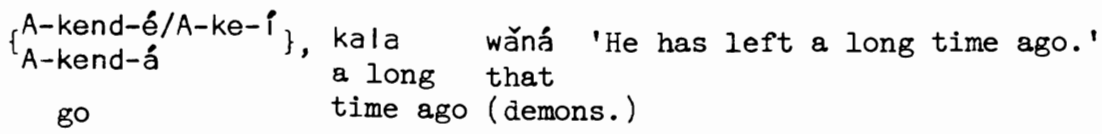

$$
\begin{aligned}
& \left\{\begin{array}{l}
(A-k e n d-e ́) / A ́-k e-i \\
* A-k e n d-a ́
\end{array}\right\} \text { káka sika óyo 'He left very recently.' }
\end{aligned}
$$

The above examples (47-48) have an opposition similar to that between the forms with $-a k^{\prime}-i$ and $-a ́ k-a ́$. The $-R+a ́ \#$ is marked for the remote origin of the state, while the construction $-\mathrm{R}+\mathrm{i} \#$ designates recent origin by conversational implicature, although it may be used arbitrarily to refer to any sort of origin for the state. 
The following examples show that these perfects may also refer to past or future states:

$$
\begin{aligned}
& \text { Ntăngo }\left\{\begin{array}{l}
\text { boyákí } \\
\text { bokoyá }
\end{array}\right\} \text { ba-ke- } i \text { 'When you }\left\{\begin{array}{l}
\text { cAme, they had left. } \\
\text { come, they will have left. }
\end{array}\right. \text { ' } \\
& \text { Ntăngo }\left\{\begin{array}{l}
\text { boyákí } \\
\text { bokoyá }
\end{array} \quad\right. \text { ba-kend-á } \\
& \text { 'When you } \left.{ }_{\text {cAme, they had left (a long time ago) }}^{\text {came, they will have left (a long time ago) }}\right\}^{\prime}
\end{aligned}
$$

Both (49) and (50) show that the state of being away was already in effect when the situation of arriving took place. Thus it even becomes questionable to say that this perfect is present or designates PRESENT RELEVANCE. It would seem more plausible to hypothesize that the perfect is neutral with respect to the opposition past, present and future, and that it designates a specific time by contextual implicature. Thus it should be added that contextual implicature has compelled a translation of (1-4), (10-13), and (46) as well as (47) in terms of PRESENT RELEVANCE. Were (46) to be substituted for bakei/bakendá in (49-50), their translations by the English present perfect would be ungrammatical. It seems plausible to me to assume that the absence of a more specifically asserted time reference to match the perfect compels us to interpret it in relation to the time of the speech event, the present (by implicature).

The next question I want to mention concerns the place of present tenses. Forms like (24-25) may refer to future too. And in (51) we observe that the perfect with $-\mathrm{R}+\mathrm{i} \#$, as well as the present durative, may refer to future:

$$
\begin{aligned}
& \text { a. Na-ke-i lelo/lobí } \\
& \text { b. Na-zal-i kokende leló/lobi > Năkokende leló/lobi } \\
& \text { 'I am leaving/will leave today/tomorrow.' }
\end{aligned}
$$

(The case of the perfect may strengthen the suspicion that $-R+i \#,-R+a ̈ \#$ forms are neutral with regard to the opposition past vs. present vs. future.) The whole set (24-25) and (51) seems to lead to the hypothesis that there may be a broad (non-punctual) concept of present, which steps already into the future. And in the absence of a time adverbial to specify the future, the present is contextually implied (if it felicitously corresponds to a concomitant situation).

The designation of some specific temporal inflections by implicature seems to be quite a generalized practice in Lingala.

4. Synthesis and Conclusion

Now let's put everything together and fill in some of the gaps in my hypotheses.

DURATIVE and GENERIC can both refer to habits. The latter is marked and can do it without the use of a complementary time adverbial while the 
DURATIVE, being the unmarked form of CONTINUITY (of a state or activity) over a long period of time, designates a habit only if complemented with a time adverbial specifying a span of time long enough to signify a habitual/ generic extension of a situation (?through recurrence). These two time reference expressions, with the third which I will subordinate to GENERIC in my table below, and which I will call PROSPECTIVE GENERIC (equivalent to Bwantsa's (VI) and (XIII)), will constitute a special class of temporal inflections which I suggest should be called CONTINUOUS. They all share the characteristic of expansion in time. The difference between the habits expressed with the durative and those expressed with the generic--besides the issue of scope discussed above--is the fact that the generic somehow commits the speaker to a prediction of the future, while the durative does not. We may also add that the extra presence of the time adverbial in (15') reduces this commitment.

A second group of temporal inflections consists of those that may be referred to as [-CONTINUOUS]. They subdivide into two subgroups: ( $i$ ) I will call the first subgroup PERFECT or STATIVE by virtue of the fact that these forms specify (a) a state which results from an asserted situation. This consists of the RESULTATIVES with $-R+i \#$ and $-R+a \#$. (b) the end of a previous situation and implication of a new state, as in the expressions with -tik- and -sil- illustrated in (31-32). A VOLITIONAL TERMINATIVE temporal inflection is designated in (32), while (31) expresses a TERMINATION unmarked with regard to volition. (The unmarked expression is more commonly used and I do not know of any conversational implicature applying here which would subject -sil- expressions to designate non-volition.) (c) the beginning of a new situation or state, the INGRESSIVE expression, with the auxiliary -kóm-, as illustrated in (33).

Here I would like to state explicitly as per the tree below, but not illustrated, that the auxiliaries -sil-, -tik- and -kóm- (like the auxiliary -zal- for the durative) are time-inflected RESULTATIVE (COINCIDENT-relevant) or SUBSEQUENT. This in fact goes together with the discussion of the questioned PRESENT-relevance above. This not only leaves an unsolved branching problem for the PERFECT in the tree, but also raises the question of what combinatorial principles are at play here. I leave the question for future investigation.

(ii) The second subgroup of [-CONTINUOUS] inflections will be referred to as PERFECTIVE by virtue of their locating the described situations without any concern for their internal temporal structures. They divide into PRIOR to, COINCIDENT with and SUBSEQUENT to the time of either the speech event or another situation specified in the utterance 
(by contextual implicature). 2 (The PERFECTIVE COINCIDENT with -a\# steps apparently into the future. Probably it should be derived from an intermediary node common with SUBSEQUENT, contrary to what is given in the tree. I leave the question unresolved for the present.)

I also observe (after Bwantsa) that something seems to be common with the dichotomy REMOTE vs. RECENT/NEAR for anything either referring to past or suggesting the past origin of a state: there is always the presence of an -a\# or -i\# associated with the particular case. We may thus safely dissociate these morphemes from the high tone -ák- of PERFECTIVE (PRIOR) and consider -ák- as corresponding to this PERFECTIVE (PRIOR) while the pair -á/-i helps to implicate NEARNESS. I am not sure I have enough evidence yet to analogize the same treatment to the UNIVERSAL GENERIC; viz. whether -aka\# or -ak+a\# should be the proper morphological division. Right now I do not see what explanatory impacts the choice of either of them would have on the theory developed here.

The table/tree on the next page summarizes my theory of Lingala temporal inflections. It is my belief that such a representation opens the way to explanation of certain oddities, particularly the selectional constraints holding between the verbal forms and the time adverbials, as seen in $(40,41,45$ and 48). A verb temporally inflected as CONTINUOUS can co-occur in the same utterance with an adverbial designating an extended span of time. A verb temporally inflected as PRIOR (REMOTE) selects an adverbial that designates a relatively remote priority/anteriority in time.

My analysis seems to give primacy to the features traditionally associated with aspect over those associated with tense, which reverses the position assigned to them in the previous analyses. First, I do not think the dichotomy "aspect vs. tense" needs to be maintained. Second,

2 There may be a problem as to what specifies the time of the situation taken as the landmark. By the very fact that there is nothing else that can specify it, the situation is to be understood in relation to the time of the speech event. This explains how the understandings of (49-50) work.

There might be an alternative interpretation of maintaining the features PAST, PRESENT and FUTURE, but this calls for an additional artificial problem of embedding either PRESENT and FUTURE into PAST or PAST and PRESENT into FUTURE, etc., as suggested by McCawley. I say "artificial" in this case because it seems to me that in (49-50) there does not apply any grammatical time reorientation, which characterizes the English taxis--about which McCawley comments as inflected in relation to both the speech event and the related situation. 
the analysis does not seem to conflict with other diachronic and comparative evidence: (a) according to Miller/Johnson-Laird [1976:449], aspectual rather than tense oppositions between situations are acquired first by the child. (b) According to Comrie [1976:83] in Indo-European, aspect distinctions seem to have occurred before tense distinctions. He also reports [op. cit: 82] that Yoruba and Igbo seem to have primacy of aspect features over tense features. Then Lingala would not be an isolated case, but would, instead, conform to a universal principle.

Many other questions need to be considered. One such may concern the hierarchy of the inflectional features I have proposed and their representation in the logical structure of sentences. Another question may specifically concern how the features which surface as adverbials and those that surface as inflectional morphemes and/or other combinations of morphemes are distributed in the logical structure. The latter question is in fact related to the one raised by Chafe [1970:168], viz., whether temporal inflection modifies the verb or the whole event described by the sentence. The limitation of time and space do not allow me to discuss these questions here.

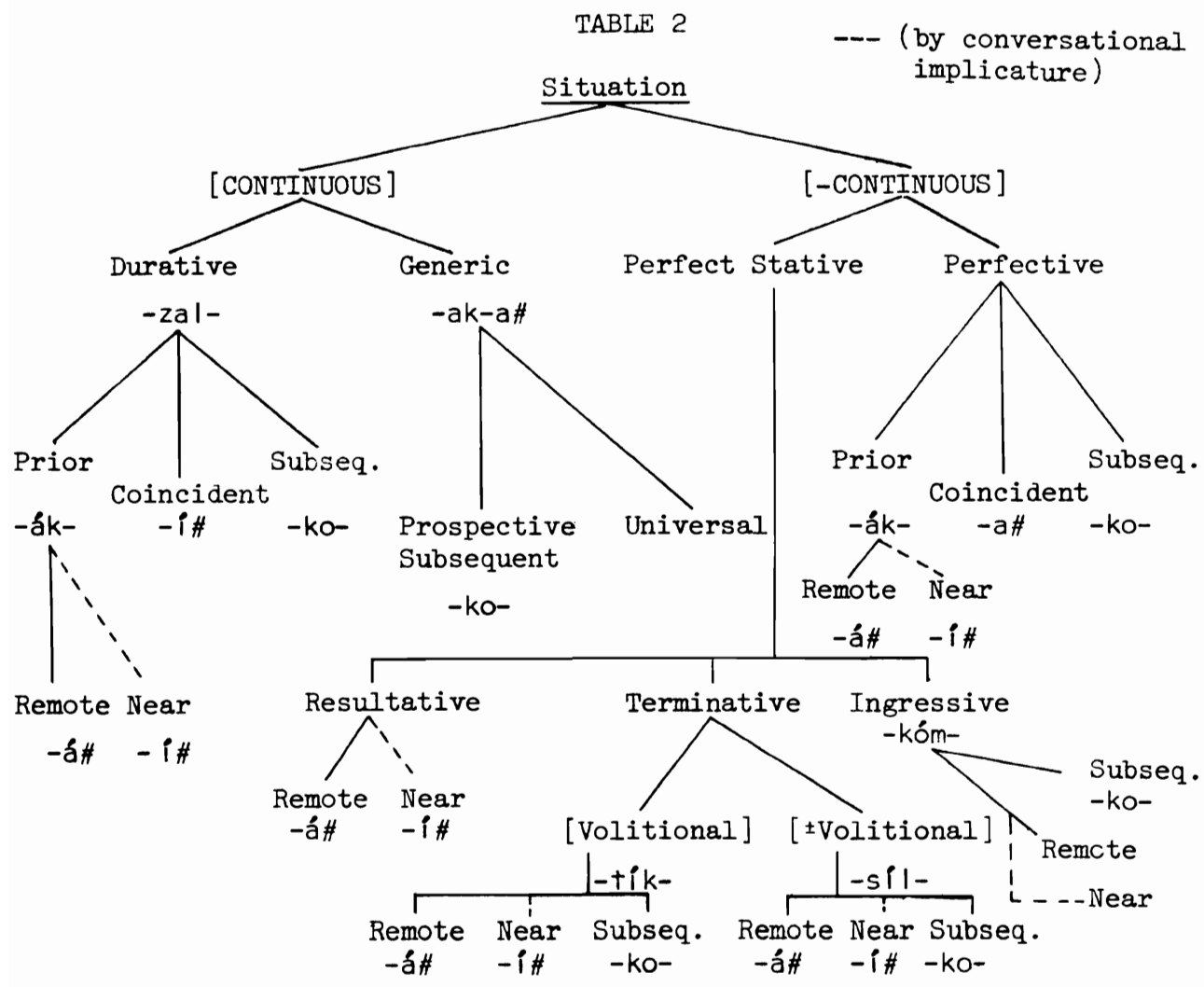


Bwantsa-Kafungu, S. Pierre. 1970. Esquisse Grammaticale de Lingala. Kinshasa: Publications de I'Université Lovanium.

Chafe, Wallace I. 1970. Meaning and the Structure of Language. Chicago: The University of Chicago Press.

Comrie, Bernard. 1976. Aspect. Cambridge: Cambridge University Press.

Guthrie, Malcolm. 1966. Grammaire et Dictionaire Lingala. Hants: Gregg Press Limited (reimpression).

Huddleston, R. 1969. "Some observations on tense and deixis in English. Language $45: 4$

McCawley, James D. 1971. "Tense and time reference in English." In Charles J. Fillmore and D. Terence Langendoen (eds.), Studies in Linguistic Semantics, pp. 96-113. New York: Holt, Rinehart and Winston.

Miller, G. A. and P. N. Johnson-Laird. 1976. Language and Perception. Boston: Harvard University Press.

Redden, James, F. Bongo et al. 1963. Iingala Basic Course. Washington D. C.: Foreign Service Institute. 



\title{
LOCATIVE PHRASES AND ALTERNATIVE CONCORD IN TSHILUBA
}

\author{
Susan U. Stucky \\ University of Illinois
}

\section{Introduction}

Locative phrases have been a subject of controversy since the beginning of grammatical analysis of Bantu languages. 1 Traditional grammars have attributed to them functions ranging from that of subject and direct object to that of adverb. Recently, Trithart [1975], Dalgish [1976a, 1976b], and Dalgish and Scheintuch [1976] have discussed locative noun phrases in various Bantu languages with respect to the grammatical relations these phrases bear to verbs. This paper continues the investigation of Bantu locatives in Tshiluba (a Zone I language spoken primarily in south central Zaire) within that cluster of hypotheses known as relational grammar. In particular, the paper focuses on two types of constructions, each of which contains a locative morpheme (either pa 'on', ku 'at', or mu 'in') followed by both a noun and a modifier which may be either an adjective or a demonstrative. Example (1) below illustrates these constructions. ${ }^{2}$ Note in (1) that the locative pa 'at' is followed by a noun mesa 'table', which in turn is followed by the adjective -nene 'big', with the concordial morpheme pa- prefixed to it. In (1b), on the other hand, the adjectival concord marker is ma- in agreement with the noun itself. The concord for locative phrases containing demonstra-

1The research for this paper has been supported by an NDFL Title VI fellowship through the University of Illinois African Studies Center. Special thanks are due to Dr. Victor C. Uchendu and the African Studies Center for funds made available to attend the VIIIth African Linguistics Conference. I would like to acknowledge the assistance of the language consultant, Mutombo Mpanya, with whom I have been working for three years. I would also like to thank Prof. Eyamba Bokamba, Prof. Charles Kisseberth, and Prof. Jerry Morgan for their helpful comments. Any errors are solely my responsibility.

${ }^{2}$ Due to the limitation of space, the number of examples is extremely limited. The handout at the conference contained more examples, which are available on request from the author. Data in an earlier version [Stucky 1976] are less complete. 
tives is contrasted in $(1 c)$ and $(1 d):{ }^{3}$
(1)
a. pa m-esa p-3-nene
on table LC-big.
'the big space on the table'
c. pa m-esa a-pa
on table this-LC
'on this table (and not off it)'
b. pa m-esa ma-nene
on table NC-big
'on the big table'
d. pa m-esa a-a
on table this-NC
'on this table (and not on
that one)'

Phrases bearing locative concord are referred to as LC phrases while those exhibiting nominal concord are called NC phrases. This phenomenon, henceforth alternative concord, not unique to Tshiluba, has been noted in ChiBemba by Givón [1972] and in OluTsootso by Dalgish (personal communication).

In addition to these alternative concordial processes, the data in this analysis show that LC and NC phrases demonstrate different syntactic behavior. Furthermore, a syntactic as well as a semantic distinction must be made between those locative phrases with adjectives and those with demonstratives. Finally, the acceptability of LC pirases depends crucially on the type of verb in the sentence.

It will be helpful to keep in mind certain facts of Tshiluba gramar. First, noun class prefixes govern concord of two types: primary, that taken by adjectives and participles, and secondary, taken by demonstratives and all other forms requiring agreement. The distinction is evident in the concordial markers in ( $1 b)$ and (Id) above. Thus, one morphological distinction relevant to the analysis of locative phrases exhibiting alternative concord can be noted at the outset. Adjectives take primary concord while demonstratives take secondary concord. Second, the locative morphemes include neither the notion of deixis, handled by the demonstratives nor that of motion to and from, which is included in the verb. Finally, unlike other noun class prefixes, the locative morphemes may appear

${ }^{3}$ Symbols are as follows: noun class numbers are not marked, but a hyphen separates a noun prefix from the stem. Ag=agreement, $O P=o b j e c t$ prefix, LP=locative pronoun, Pass=passive, Refl=reflexive marker, LC= locative concord, NC=noun concord. Standard orthography has been employed here with one exception. Long vowels that are not derived by rule are written as sequences of two short vowels. Tone is not marked. (See footnote 4 for tone rules.) 
before full nouns. ${ }^{4}$

\section{Syntactic Properties of LC and NC Locative Phrases}

Besides the agreement contrast noted in the previous section, LC and NC phrases behave differently with respect to certain syntactic processes. A semantic distinction also emerges. One restriction on alternative concord is, however, noted first. Example (2) below shows that alternative concord does not extend to the predicate but is entirely restricted to the locative phrase. Verbal agreement is always with the locative.
(2) a. pa m-esa pa-di pa-nene
on table $\mathrm{Ag}$-be $\mathrm{Ag}-\mathrm{big}$
'on a/the big table'
b. *pa m-esa ma-di pa-nene
on table Ag-be Ag-big
c. *pa m-esa pa-di ma-nene on table $\mathrm{Ag}$-be $\mathrm{Ag}$-big
d. *pa m-esa ma-di ma-nene on table $\mathrm{Ag}$-be $\mathrm{Ag}$-big

Syntactic processes illustrating the difference between LC and NC phrases include the non-relation changing rules of left and right dislocation. The data in (3) and (4) below show that the LC phrase is more tightly knit than the NC phrase. Example (3a) shows that either a LC or a NC phrase may be left-dislocated by leaving a pronoun behind, suffixed to the verb. Alternatively, in ( $3 b)$, the NP tshisalu etshi 'this market' can be left-dislocated from within a NC phrase, but not, significantly, from within a LC phrase. Example (4) shows that right-dislocation proceeds analogously.

${ }^{4}$ There is little phonological or tonological evidence in Tshiluba for considering the locative morpheme together with its noun to be a single word. No noun begins with a vowel (since there are no vowel preprefixes) and thus vowels do not coalesce. Locatives standing alone have high tone. Affixed to a stem they assimilate in tone. This does not provide any evidence either, since all noun prefixes in Tshiluba have high tone and the locatives in these phrases exhibit high tone. There is some syntactic evidence for considering them to be a single unit. No other word, such as a demonstrative, may intervene between the locative and the noun. Normally, the demonstrative may either precede or follow the noun. Still, traditional orthography has treated these as two separate words. The spelling in this paper conforms to that convention. 
(3) a. mu tshi-salu $\left\{\begin{array}{l}e-m u \\ e-t s h i\end{array}\right\}$ mu-kaji u-di w-enda- mu

in market $\left\{\begin{array}{c}\text { this-LC } \\ \text { this-NC }\end{array}\right\}$ woman Ag-be Ag-walk to- LP

'in this market, the woman is walking (to) (in) here'

b. tshi-salu $\underset{\substack{* e-m u \\ e-t s h i}}{*}$ mu-kaji u-di w-enda- mu

market $\left\{\begin{array}{c}\text { *this-LC } \\ \text { this-NC }\end{array}\right.$ woman Ag-be Ag-walk to-LP

'this market, the woman is walking (to) (in) here'

(4) a. mu-kaji u-di w-enda- mu, mu tshi-salu $\left\{\begin{array}{l}e-m u \\ e-t s h i\end{array}\right\}$

woman Ag-be Ag-walk to-LP, in market $\left\{\begin{array}{l}\text { this-LC } \\ \text { this-NC }\end{array}\right\}$

'the woman is walking (to) (in) here, in this market'

b. mu-kaji u-di w-enda- mu tshi-salu $\left\{\begin{array}{c}\text { *e-mu } \\ e-t s h i\end{array}\right\}$

woman $\mathrm{Ag}$-be $\mathrm{Ag}$-walk to-LP market $\left.\begin{array}{r}\text { *this-LC } \\ \text { this-NC }\end{array}\right\}$

'the woman is walking (to) (in) here, this market'

Unlike the dislocation rules, relativization is sensitive to grammatical relations. This process also reflects the structural distinction between LC and NC phrases. In (5) and (6) below, both LC and NC phrases are relativized. As in the case of the dislocation rules, the LC phrase must remain a syntactic unit, but the NC phrase may be relativized out of.

$$
\begin{aligned}
& \text { mu tshi-longelu } \underset{\{e-t s h i}{e-m u}\} \quad m u-d i-b o \quad \begin{array}{l}
\text { ba- bala mi-kanda } \\
\text { they-read }
\end{array} \\
& \text { in school } \left.\quad \begin{array}{c}
\text { this-LC } \\
\text { this-NC }
\end{array}\right\} \text { Rel-be-they books }
\end{aligned}
$$

'inside/in this school in which they are reading books...'

$$
\begin{aligned}
& \text { mu tshi-longelu }\left\{\begin{array}{c}
{ }^{*} e-m u \\
e-t s h i
\end{array}\right\} \text { tshi-di-bo ba- tok- esha } \\
& \text { in school } \left.\quad \begin{array}{r}
\text { this-LC } \\
\text { this-NC }
\end{array}\right\} \text { Rel-be-they they-clean-Caus. }
\end{aligned}
$$

Thus, at least three processes, right and left dislocation and relativization indicate that the LC phrase is a syntactic unit while the NC phrase may be treated either as a single unit or as two, the locative and the noun plus its modifier.

3. Alternative Concord and Grammatical Relations

3.1 Subject of. Whether or not LC and NC phrases can be subjects is a problem that requires a longer investigation than is possible here. Constructions where locatives appearing in initial position govern verbal agreement are the LOC-BE-ADJ and LOC-BE-NP constructions shown in examples 
(7) and (8) below. Here there is a clear difference between the locative adjective and the locative demonstrative phrases. That both IC and NC phrases are acceptable in LOC-BE-ADJ constructions is illustrated in ( 7 ). Thus, it is possible to predicate a quality of either LC or NC adjective phrases. In (8) however, an LC-adjective phrase is unacceptable in LOCBE-NP constructions. In contrast, both $\mathrm{LC}$ and NC demonstrative phrases are acceptable in both $\mathrm{BE}$ constructions, as is evidenced in the (a) and (b) sentences of (7) and (8). The LC-adjective ( $8 \mathrm{c})$ is interpreted as a locative noun. This is reflected in the English gloss the clean space on', rather than 'the space on the clean table'. on table this-LC Ag-be Ag-wet is wet'
a. pa m-esa a-pa pa-di pa-bole
'on this table (and not off it)
b.
c. pa m-esa pa-tooke pa-di pa-bole on table $\mathrm{LC}$-clean $\mathrm{Ag}$-be $\mathrm{Ag}$-wet 'the clean space on the table is wet'

pa m-esa

on table this

'on this table (and not that one) is wet'

d. pa m-esa ma-tooke pa-di pa-bole on table $\mathrm{NC}-\mathrm{clean} \mathrm{AG}$-be Ag-wet 'on the clean table is wet'
(8) a. pa m-esa a-pa pa-di mi-kanda b. pa m-esa a-a pa-di mikanda on table this-LC Ag-be books 'on this table (and not off it) are books'
on table this-NC Ag-be books 'on this table (and not that one) are books'
c. *pa m-esa pa-tooke pa-di mi-kanda d. pa m-esa ma-tooke pa-di mi-kanda on table LC-clean Ag-be books * 'the clean space on the table is books' on table NC-clean Ag-be books 'on the clean table are books'

The items in (7) and (8) also demonstrate that the locative morpheme always governs verbal agreement on the verb. According to one early version of relational grammar [Postal and Perlmutter 1974] one of its tenets is that only terms (i.e. subjects) can trigger verbal agreement. The fact that these LC and NC phrases govern agreement may constitute an argument in favor of termhood. However, there are at least two other explanations for this pattern of agreement. First, it may be that agreement in Tshiluba is not with a term but with a noun that is not necessarily a subject. Secondly, it could be the case that the simple noun is the term but the agreement is with the larger phrase of which the noun is a part. Thus, verbal agreement is not sufficient to determine termhood, suggesting that more evidence is needed.

Another test of termhood is reflexivization. If locatives are subjects, they should trigger reflexivization. In the following example, both LC and NC phrases trigger reflexivization. 
(9) a. mu tshi-bunda e-mu mu-di- shimbula

in garden this-LC Ag-Refl-collapse

'inside this garden (and not outside) fell in itself'

b. mu tshi-bunda e-tshi mu-di- shimbula

in garden this-NC Ag-Refl-collapse

'in this garden (and not another one) fell in itself'

Thus, if it is true that only terms can govern agreement and trigger reflexivization, then the locatives are subjects in (6-9). Whether they got there by an advancement rule (one which moves an NP up the hierarchy of grammatical relations) or a movement rule (one which does not change grammatical relations) or by being there underlyingly is an open question.

3.2 object of. The relation considered next is that of object. As in the preceding section on the subject relation, the behavior of locative phrases containing adjectives is different from those containing demonstratives.

Consider first locative phrases with adjectives illustrated by just examples (a) and (b) in (10)-(13). We see in (10) that a LC-adjective phrase--but not a NC-adjective phrase--together with an object, is unacceptable. In the same type of construction, though, LC- and NC-adjective phrases in (II) are both acceptable.

a. mu-kaji u-di u-teka mi-kanda mu tshi-longelu *mu-nene woman $\mathrm{Ag}$-be $\mathrm{Ag}$-put books in school LC-big *'the woman is putting the books the big space in the school'

b. mu-kaji u-di u-teka mi-kanda mu tshi-longelu tshi-nene woman $\mathrm{Ag}$-be $\mathrm{Ag}$-put books in school NC-big 'the woman is putting the books in the big school'

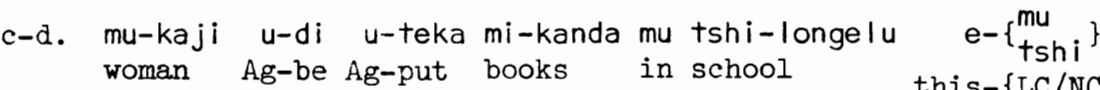

'the woman is putting books in this school' this $-\{\mathrm{LC} / \mathrm{NC}\}$

(II) a. mu-kaji u-di u-bala mi-kanda mu tshi-longelu mu-nene woman Ag-be Ag-read books in school LC-big 'the woman is reading books in the big space in the school'

b. mu-kaji u-di u-bala mi-kanda mu tshi-longelu tshi-nene woman $\mathrm{Ag}$-be $\mathrm{Ag}$-read books in school NC-big 'the woman is reading the books in the big school'

$c-d$. mu-kaji u-di u-bala mi-kanda mu tshi-longelu $e-\left\{\begin{array}{c}m u \\ +s h i\end{array}\right\}$ woman $\mathrm{Ag}$-be $\mathrm{Ag}$-read books in school this- $\{\mathrm{LC} / \mathrm{NC}\}$

Again, in (12), both LC- and NC-adjective phrases may follow the verb. However, in (13), only a NC-adjective is acceptable. 
a. mu-kaji u-di w-enda mu tshi-longelu mu-nene

woman $\mathrm{Ag}$-be $\mathrm{Ag}$-walk to in school LC-big

'the woman is walking to the big space in the school'

b. mu-kaji u-di w-enda mu tshi-longelu tshi-nene

woman $\mathrm{Ag}$-be $\mathrm{Ag}$-walk to in school NC-big

'the woman is walking into the big school'

c-d. mu-kaji u-di w-enda mu tshi-longelu e- $\left.\begin{array}{c}m u \\ +s h i\end{array}\right\}$

woman Ag-be Ag-walk to in school this-\{LC/NC\}

'the woman is walking into this school'

(13)
a. ma-futa ma-di ma-aya
mu tshi-lowu *mu-nene
oil Ag-be Ag-get rancid in calabash LC-big
* 'oil is getting rancid the big space in the calabash'
b. ma-futa ma-di ma-aya mu tshi-lowu tshi-nene
oil $\mathrm{Ag}$-be $\mathrm{Ag}$-get rancid in calabash NC-big
'oil is getting rancid in the big calabash'
c-d. ma-futa ma-di ma-aya mu tshi-lowa e- $\left.\begin{array}{c}m u \\ +s h i\end{array}\right\}$
oil Ag-be Ag-get rancid in calabash this- $\{\mathrm{LC} / \mathrm{NC}\}$
'the oil is getting rancid in this calabash'

Upon closer examination one finds that the verbs in (10-13) fall into different classes with respect to the types of NPs required or permitted to accompany them. Verbs such as -teka 'put', in (10), rarely surface without objects. Verbs such as -bala 'read', in (1l), often surface without objects. Verb requiring direct objects are here labeled strongly transitive verbs. Verbs permitting, but not requiring, direct objects are labeled weakly transitive. A verb like -enda 'walk to' in (12) belongs to another class of verbs, motion intransitives, which are distinguished from strictly intransitive verbs such as -aya 'get rancid' in (13). Motion intransitives actually require locatives while strictly intransitive verbs, although allowing them, do not require them. It appears that the LC phrase does not satisfy the requirements of a strongly transitive verb, as in (10). In (11), both LC- and NC-adjective phrases are acceptable with weakly transitive verbs. In (12), likewise, both LC- and NCadjective phrases can satisfy a motion intransitive. But only NC-adjective phrases can satisfy a strictly intransitive verb as in (13).

Locative phrases containing demonstratives rather than adjectives exhibit none of the idiosyncracies of the adjective phrases. As examples $(c-d)$ in (10-13) show, regardless of whether the demonstrative locative phrase is an LC or an NC phrase, it is always acceptable. Thus, it cannot be claimed that the type of verb determines the acceptability of LC phrases alone. Rather the type of verb and the type of locative phrase must be taken into account.

Returning to the question of locative adjective phrases, the proposition that the LC- and NC-adjective phrases differ in their capacity to 
satisfy the requirements of the verb can be tested as follows. First, with a strongly transitive verb having no object, an LC phrase should be acceptable, but an NC phrase should not. That this is indeed the case is illlustrated by the examples in (14):

$$
\begin{aligned}
& \text { a. mu-kaji u-nanga ku n-zuby ku-nene } \\
& \text { woman Ag-like at house LC-big } \\
& \text { 'the woman likes the atmosphere at the big house' } \\
& \text { b. mu-kaji u-nanga ku n-zubu *mu-nene } \\
& \text { woman Ag-like at house NC-big } \\
& \text { *'the woman likes at the big house' }
\end{aligned}
$$

With a verb such as -bala 'read', a weakly transitive verb, we might expect a LC-adjective phrase to have two readings: one a patient (where the LC-adjective phrase satisfies the requirements of transitivity) and the second a locative reading (where object deletion has applied). In fact, two readings ase obtainable for such a verb, as is shown in (15):

$$
\begin{aligned}
& \text { a. mu-ntu u-di u-songa mu bu-atu mu-nene } \\
& \text { man Ag-be Ag-carve in boat LC-big } \\
& \text { 'the man is carving out the inside of the boat--making it bigger' } \\
& \text { OR 'the man is carving (something else) in the big boat' }
\end{aligned}
$$

b. mu-ntu u-di u-songa mu bu-atu mu-nene man Ag-be Ag-carve in boat NC-big 'the man is carving (something else) in the big boat'

The NC phrase in (15b) cannot be used in the situation where buatu 'boat' is the patient or the receiver of the action. LC-adjective phrases consequently have two qualities of direct objecthood: they follow transitive verbs while $\mathrm{NC}$-adjective phrases do not, and they are interpretable as patients.

Next, consider the two cases where a verb actually requires a certain kind of NP. As noted above, a LC phrase in (10a), following a strongly transitive verb and its attendant object, was unacceptable. This behavior may be attributed to the fact that the LC-adjective phrase behaves like an object. Since the verb requires only one object, the LC-adjective phrase is then superfluous. Motion intransitives, like strongly transitive verbs, require a NP, a locative in this case. A NP not marked morphologically as a locative will not suffice, as example (16) illustrates:

$$
\begin{array}{ccc}
\text { * mu-kaji } u \text {-di w-enda } & n \text {-zubu } \\
\text { woman } & \mathrm{Ag} \text {-be Ag-walk to house }
\end{array}
$$

A LC-adjective phrase, as noted above, could satisfy motion intransitives. Although the addition of a second LC-adjective phrase is not acceptable, the presence of a second NC-adjective phrase is. As in the case of strongly transitive verbs, the LC-adjective phrase is unacceptable. Compare examples $(17 \mathrm{a})$ and $(17 \mathrm{~b})$ : 


$$
\begin{aligned}
& \text { b. mu-kaji u-di w-enda mu tshi-longelu mu-flike ku } \\
& \text { woman } \mathrm{Ag} \text {-be } \mathrm{Ag} \text {-walk to in school LC-dark at } \\
& \text { mu-soko }\left\{\begin{array}{c}
\text { ku- lenga } \\
\text { mu- }
\end{array}\right. \\
& \text { village }\{\stackrel{\text { LC- }}{\mathrm{NC}-} \text { beautiful }\}
\end{aligned}
$$

Thus, the acceptability of LC-adjective phrases, but not LC-demonstrative phrases, is seen to be directly related to the type of verb, whether strongly or weakly transitive, a motion intransitive or a strictly intransitive verb. The LC-adjective phrase behaves more like a direct object while still being marked as a locative by the locative prefix and its objecthood is paralleled by its nounlike reading.

3.3 Pronominalization. There are two cases (1la and 1lb) where a LC-adjective phrase exhibited some properties normally associated with direct objecthood that are not yet accounted for. LC-adjective phrases in (15a and 15b), following transitive verbs, were interpretable as patients. It seems prudent, then, to investigate some syntactic processes that might reflect their status. The first is pronominalization. Here an interesting correlation appears between the position of the locative pronoun and the appearance of LC phrases in such sentences as shown in (18a) and (19a) below. Normally two positions are available for object pronouns, preceding the verb stem and following it. If only a direct object is present, the pronoun appears in prefix position. Note that in (18a)--corresponding to (15a)--and in (19)--corresponding to (14)--the locative pronoun shows up in prefix position as a direct object would. The non-object reading of the LC phrase--corresponding to (15a)--and the NC phrase pronoun appear in suffix position as illustrated in $(18 \mathrm{~b})$. The examples are given in terms of dislocation so that the pronoun can be checked most accurately.

(18) a. mu bu-atu mu-nene mu-ntu u-di u-mu-songa

in boat LC-big man Ag-be $\mathrm{Ag}-\overline{\mathrm{LP}}$-carve

'the big space in the boat, the man is carving it'

b. mu bu-atu $\left\{\begin{array}{l}m u-n e n e \\ b u-\end{array}\right.$ mu-ntu u-di u-songa-mu

in boat $\quad\left\{\begin{array}{l}\mathrm{LC}-\mathrm{big} \\ \mathrm{NC}-\end{array}\right.$ man Ag-be Ag-carve-LP

'in the big boat, the man is carving it'

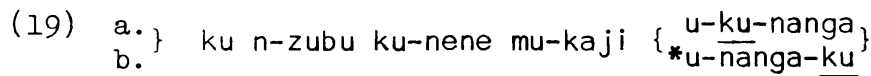

at house LC-big woman $\left\{\begin{array}{c}\mathrm{Ag}-\mathrm{LP}-\mathrm{like} \\ { }^{*} \mathrm{Ag}-\mathrm{like-LP}\end{array}\right\}$

'at this big house, the woman likes it there'

The LC-adjective and NC-adjective pronouns with -bala 'read', a weakly transitive verb, evince a clear preference for suffix position even when no other object is present, as the following examples illustrate: 

verbs. These do not passivize. Compare (24-28) below:

(24) ku n-zubu e-ku ku-nang-ibue kudi mu-kaji

at house this-LC Ag-like-Pass by woman

'the atmosphere at this house is liked by the woman'

(25) mu bu-atu mu-nene mu-song-ibue kudi mu-ntu

in boat LC-big Ag-carve-Pass by man

'the space inside of the boat was carved bigger by the man'

(26) mu tshi-longelu $\left\{\begin{array}{l}m u- \\ \text { tshi-nene }\} \text { mu-bad-ibue kudi mu-kaji }\end{array}\right.$

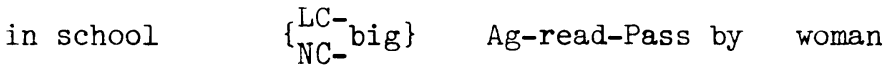

'in the big school was read by the woman'

(27) mu tshi-salu $\left\{\begin{array}{l}m u- \\ \text { tshi-nene }\end{array}\right\}$ mu-end -ibue kudi mu-kaji

in school $\quad\left\{\begin{array}{l}\mathrm{LC}-\text { big } \\ \mathrm{NC}-\end{array} \quad \mathrm{Ag}\right.$-walk to-Pass by woman

'in the market was walked to by the woman'

(28) *mu tshi-lowa tshi-nene mu-ay -ibue kudi ma-futa

in calabash NC-big Ag-got rancid-Pass by oil

*'in the calabash was gotten rancid by the oil'

The last restriction on passivization indicates that it is not sufficient to speak of which items may passivize. Instead, in the case of strictly intransitive verbs it is necessary to consider whether or not the locative was required by the verb in the first place.

4. Summary

The data in this paper have shown that the category locative is not a discrete one in Tshiluba. The morphological distinction evidenced through alternative concord is reflected in three syntactic processes: pronominalization, passivization and relativization. Semantically, LC phrases focus the locative while NC phrases focus the noun. Furthermore, LC and NC phrases are distinguished along another parameter, modification, where the modifier is either an adjective or a demonstrative.

With respect to grammatical relations, it was argued on the basis of verbal agreement and reflexivization that locatives could occur as underlying subjects, terms. It was also noted that these locatives have several properties of objecthood; they may pronominalize and passivize. However, the ability to passivize, it was shown, did not depend on the locative itself, but rather, on the type of verb. Furthermore, LC and NC phrases appear to be on a continuum. The LC-adjective phrase is more like a direct object, since only it may satisfy the requirements of a strongly transitive verb.

Finally, locatives in Tshiluba must be distinguished from other objects, since both may appear simultaneously and have separate positions for pronouns. Motion intransitives require locatives in the same way 
Dalgish, Gerard M. 1976a. "Locative NPs, locative suffixes and grammatical relations." Proceedings of the Second Meeting of the Berkeley Linguistic Society. pp. 139-148.

Dalgish, Gerard M. 1976b. "Passivizing locatives in OluTsootso." Studies in the Linguistic Sciences. 6.1:57-68.

Dalgish, Gerard M. and Gloria Scheintuch. 1976 "On the justification for language-specific sub-grammatical relations." Studies in the Linguistic Sciences. 6.2:89-107.

Givón, Talmy. 1972. Studies in ChiBemba and Bantu Grammar. Studies in African Linguistics. Supp. 3 .

Keenan, Edward L. 1976. "Toward a universal definition of 'subject'." In Charles N. Li (ed.), Subject and Topic. pp. 303-333. New York: Academic Press.

Keenan, Edward L. and Bernard Comrie. 1977. "Noun phrase accessibility and universal grammar." Linguistic Inquiry. 8.1:63-99.

Perlmutter, D. and Paul M. Postal. 1974. "Some general laws of grammar." Handout, Linguistics Institute.

Stucky, Susan U. "Locatives as objects in Tshiluba: a function of transitivity." Studies in the Linguistic Sciences. 6.2:174-202.

Trithart, Lee. 1975. "Relational grammar and ChiChewa subjectivization rules." In Proceedings of the Eleventh Regional Meeting of the Chicago Linguistic Society, pp. 615-624. Chicago: Chicago Linguistic Society. 